To be submitted to The Astrophysical Journal

\title{
Formation Rates of Black Hole Accretion Disk Gamma-Ray Bursts
}

\author{
Chris L. Fryer, S. E. Woosley \\ Astronomy Department, University of California \\ Santa Cruz, CA 95064 \\ cfryer@ucolick.org \\ Dieter H. Hartmann \\ Clemson University \\ Clemson, SC, 29634
}

\begin{abstract}
The cosmological origin of at least an appreciable fraction of classical gamma-ray bursts (GRBs) is now supported by redshift measurements for a half-dozen faint host galaxies. Still, the nature of the central engine (or engines) that provide the burst energy remains unclear. While many models have been proposed, those currently favored are all based upon the formation of and/or rapid accretion into stellar mass black holes. Here we discuss a variety of such scenarios and estimate the probability of each. Population synthesis calculations are carried out using a Monte Carlo approach in which the many uncertain parameters intrinsic to such calculations are varied. We estimate the event rate for each class of model as well as the propagation distance for those having significant delay between formation and burst production, i.e., double neutron star (DNS) mergers and black hole - neutron star (BH/NS) mergers. One conclusion is a one to two order of magnitude decrease in the rate of DNS and BH/NS mergers compared to that previously calculated using invalid assumptions about common envelope evolution. Other major uncertainties in the event rates and propagation distances include the history of star formation in the universe, the masses of the galaxies where merging compact objects are born, and the radii of the hydrogen stripped cores of massive stars. For reasonable assumptions regarding each, we calculate a daily event rate in the universe for i) merging neutron stars: $\sim 100 /$ day; ii) neutron-star black hole mergers: $\sim 450$ /day; iii) collapsars: $\sim 10^{4}$ /day; iv) helium star black hole mergers: $\sim 1000 /$ day; and v) white dwarf black hole mergers: $\sim 20 /$ day. The range of uncertainty in these numbers however, is very large, typically two to three orders of magnitude. These rates must additionally be multiplied by any relevant beaming factor $\left(f_{\Omega}<1\right)$ and sampling fraction (if the entire universal set of models is not being observed). Depending upon the mass of the host galaxy, half of the DNS mergers will happen within $60 \mathrm{kpc}$ (for a galaxy with a mass comparable to that of the Milky Way) to $5 \mathrm{Mpc}$ (for a galaxy with negligible mass) from the galactic center. The same numbers characterize BH/NS mergers. Because of the delay time, neutron star and black hole mergers will happen at a redshift 0.5 to 0.8 times that of the other classes of models. Information is still lacking regarding the hosts of short hard bursts, but we suggest that they are due to DNS and BH/NS mergers and thus will ultimately be determined to lie outside of galaxies and at a closer mean distance than long complex bursts (which we
\end{abstract}


attribute to collapsars). In the absence of a galactic site, the distance to these bursts may be difficult to determine.

Subject headings: gamma-rays: bursts - binaries: close - black holes

\section{Introduction}

The mechanisms responsible for gamma-ray bursts (GRBs) are not yet known with any certainty, but as evidence for a cosmological origin mounts (e.g., Metzger et al. 1997; Frail et al. 1997; Bloom, et al. 1998; Bloom et al. 1999a), models based upon black hole accretion disks (BHAD models) have gained prominence. Such models have several advantages, especially their large inherent energy and potentially short time scales. In the neutrino-powered GRB paradigm, BHAD models can overcome the strong angle dependence of neutrino annihilation and extract some of the gravitational energy of the disk, possibly without ejecting too much baryonic matter (Paczynski 1991; Woosley 1993; Ruffert et al. 1997; Popham, Woosley, \& Fryer 1998; MacFadyen \& Woosley 1999). BHAD models also provide favorable conditions for magnetic fields to extract energy, either from the gravitational potential energy of the accretion torus itself, or from the rotational energy of spinning black holes (Blandford \& Znajek 1977; MacDonald et al. 1986;

Paczynski 1991, 1998; Narayan et al. 1992; Hartmann \& Woosley 1995; Meszaros \& Rees 1997a; Katz 1997; Livio, Ogilvie, \& Pringle 1999). The merger of double neutron star (DNS) systems is probably the best studied representative of this class, but a variety of progenitor systems lead to similar conditions: mergers of other compact systems such as binaries consisting of a neutron star and a black hole (BH/NS) or of a white dwarf and black hole (WD/BH), the inspiral of a compact object into its companion's helium core during common envelope evolution (helium-mergers), and the accretion of material into a black hole in a "failed supernova" (collapsar).

Estimates of the rates for these various occurrences are plagued by a variety of uncertainties in the supernova explosion mechanism, stellar evolution, and binary evolution. Although the current literature abounds with population synthesis calculations, especially of DNS systems, and the closely-related BH/NS binaries (Clark, van den Heuvel, \& Sutantyo 1979; Lipunov, Postnov, \& Prokhorov 1987; Hills, Bender \& Webbink 1991; Tutukov \& Yungelson 1993; Lipunov et al. 1995; Portegies-Zwart \& Spreeuw 1996; Fryer, Burrows, \& Benz 1998; Portegies-Zwart \& Yungelson 1998, Bethe \& Brown 1998; Belczyński \& Bulik 1998; Bloom, Sigurdsson, \& Pols 1999b), little attention has been directed towards either exploring the uncertainties in the models or in examining the other kinds of models.

In this paper, we shall study the rate of formation of all varieties of BHAD progenitors, including our best estimates of the (admittedly uncertain) physics. We will show that the discrepancy between rates calculated from population studies and extrapolations from the data of DNS systems are reconcilable if one includes both the uncertainties in the population synthesis calculations and the error in the measured rates. BHAD models other than DNS will also be considered within the same parameterized framework. Comparisons with observed compact systems can be used to constrain some of the parameters, e.g., the pulsar velocity distribution and the observed properties of X-ray binaries and DNS systems. However, even with these constraints, we shall find that most rates for GRB progenitor formation are uncertain by over two orders of magnitude.

From these studies, we shall also determine the velocity distributions of the GRB progenitor systems and their corresponding merger time scales. By evolving these systems in representative gravitational 
potentials for both high and low mass galaxies, we can determine the distances each GRB progenitor travels before producing a GRB. A fraction of the compact binary systems receive large enough systemic velocities to be ejected from their host galaxies, but many remain bound even in such low mass galaxies as the host of GRB970508 (Bloom et al. 1998).

In $\S 2$, we describe formation scenarios for each of the black hole accretion disk GRB progenitor systems. Section 3 describes our population synthesis code and the parameters of population synthesis, noting the effects of the uncertainty in each. Section 4 gives our predictions, including the effects of varying systemic velocities and merger time scales to obtain both the spatial and temporal distribution of GRBs from each progenitor. We conclude with a summary of the merits of the different models.

\section{Black Hole Accretion Disk progenitors}

Modeling the progenitors of BHAD derived GRBs involves all the uncertainties inherent in any study of compact object formation. Moreover, since the systems must be in short-period binaries to merge, the problem is compounded by uncertainties in the parameters of binaries (mass ratios, separations) and their evolution. Before delving farther into these uncertainties, it is first helpful to have in mind some general scenarios (Table 1, Fig. 1).

\subsection{Double Neutron Star Binaries}

When two neutron stars merge, they quickly form an object too large to be supported by nuclear and degeneracy pressure (for most choices of equation of state). A black hole forms on a dynamic time scale, but a significant amount of mass, typically $0.03-0.3 \mathrm{M}_{\odot}$, has too much angular momentum to enter the hole promptly. An accretion disk forms. What happens next depends upon the viscosity and entropy of this disk, the evolution of its magnetic field, and the interaction of the disk with the rotating black hole. All three may be quite complicated. If the viscosity is low and the temperature inadequate to drive rapid energy loss by neutrinos, the disk may persist for a long time, perhaps over $10 \mathrm{~s}$ in the models envisioned by Meszaros \& Rees (1997a). Any relativistic outflow that develops must then be a consequence of MHD processes in the disk or of the magnetic extraction of rotational energy from the hole. These same MHD processes can also function in a viscous disk (indeed some appreciable magnetic field must be present to provide the viscosity), but the time scale is much shorter and the temperature higher. Most models of DNS mergers that have been carried out on the computer are of this latter variety (Davies et al. 1994; Rasio \& Shapiro 1994; Ruffert, Janka, Schäfer 1995; Janka \& Ruffert 1996; Wilson, Mathews, \& Marronetti 1996; Mathews \& Wilson 1997; Ruffert et al. 1997, Ruffert \& Janka 1998; Rossweg at al. 1999). In the "hot model" The entire accretion process then lasts for 10-100 ms and most of the binding energy of the disk is radiated as neutrinos. Indeed the neutrino luminosity can be so great that a significant fraction, on the order of $1 \%$, is converted, via neutrino annihilation, to thermal energy along the rotational axis. This is enough to power energetic jets that produce a GRB either by internal or external shocks. The hot DNS model might therefore be more appropriate for the short, hard subset of GRBs (Fishman \& Meegan 1995) which are very difficult to make other ways (except in the closely allied BH/NS model). Observationally, these short hard bursts are not yet constrained, either in energy or host, by the observations of Beppo-Sax, which hitherto has employed a 5 s trigger time.

The progenitor systems here are double neutron stars in binaries so close that merger occurs in a 
Hubble time as a consequence of gravitational radiation. DNS systems have a long observable lifetime because the primary's neutron star accretes matter and becomes a recycled radio pulsar. Recycled pulsars are thought to be produced in binaries where accretion from a companion spins up the neutron star whose magnetic field has partially decayed (see reviews by Verbunt 1993; Phinney \& Kulkarni 1994). Due to the low magnetic field strengths, spin down of these pulsars occurs slowly, and consequently recycled radio pulsars have observable lifetimes $\gtrsim 10-100$ times longer than normal pulsars. It is not surprising then that essentially all pulsars observed in double neutron star systems are recycled pulsars.

The standard scenario for forming close orbit DNS systems (Scenario I, Fig. 2) begins with two massive stars. The more massive star (primary) evolves off the main sequence, overfills its Roche-lobe, and transfers mass to its companion (secondary). The primary then evolves to the end of its life, forming a neutron star in a supernova explosion. The binary system remains bound, unless large kicks neutron star during formation, and a binary consisting of a neutron star and massive companion is formed. This system passes through an X-ray binary phase which then evolves through a common envelope as the secondary star expands. During this common envelope phase in the standard model, the neutron star spirals into the massive secondary and the orbital energy released ejects the secondary's hydrogen mantle, forming a neutron star/helium star binary. Mass accretion onto the neutron star primary is alleged to add angular momentum to the neutron star, "recycling" it as a pulsar. After the explosion of the helium star as a supernova, a close DNS binary remains which, in time, merges via gravitational wave emission.

However, recent calculations of neutron star accretion (Chevalier 1993, 1996; Houck \& Chevalier 1992; Brown 1995; Fryer, Benz, \& Herant 1996) reveal that, during the common envelope phase, the neutron star accretes at the Bondi-Hoyle rate, not the Eddington rate. During its spiral in, the neutron star can accrete over a solar mass (Bethe \& Brown 1998) and collapse to form a black hole. Thus, the "standard" scenario for DNS systems may in fact form BH/NS binaries.

How, then, are close DNS systems formed? Brown (1995) suggested an alternate scenario (Scenario II, Fig. 3) in which the initial binary system is comprised of two massive stars of nearly equal mass. The secondary (whose mass is assumed to be within $5 \%$ of the primary) evolves off the main sequence before the explosion of the primary as a supernova. The two stars then enter a common envelope phase with two helium cores orbiting within one combined hydrogen envelope. After the hydrogen envelope is ejected, first one, then the other, helium stars explode. If the kicks imparted to the neutron stars during the supernova explosion are sufficiently low, a double neutron star system can form. To generate the observed systems, after the first supernova, the newly formed neutron star must accrete material, possibly from its companion's helium star wind, to become the observed "recycled" radio pulsar. If accretion is insufficient, a "silent" double neutron star binary is formed in which neither neutron star is recycled. Such systems, though only briefly detectable as radio pulsras, might still evolve to produce GRBs.

A third scenario (Scenario III, Fig. 4) avoids any common envelope phase, but requires that the neutron star formed in the explosion of the secondary receive a kick that places it into an orbit that will allow the two neutron stars to merge within a Hubble time. However, in general, to avoid a common envelope phase, the pre-supernova orbital separation must be extremely wide (greater than $1 \mathrm{AU}$ ). The range of kick magnitudes and directions which will produce such orbits is so small that, as we shall show in this paper, this formation scenario is probably not important.

\footnotetext{
${ }^{1}$ The pulsar velocity distribution requires that neutron stars are somehow accelerated to mean velocities of roughly $\sim 450 \mathrm{~km} \mathrm{~s}^{-1}$, most likely at or near the time of birth. We discuss this in more detail in $\$ 3.2$.
} 
From the number of known Galactic DNS binaries (PSR 1913+16, PSR 1534+12), a formation rate can be estimated. However, correcting for time evolution of pulsar luminosities, beaming fractions, and distance estimations proves tricky for a sample of only two merging double neutron star systems. From the number of observed DNS systems $\left(N_{\text {obs }}\right)$, and including an estimate of all the selection effects: beaming fraction, fraction of galaxy currently observed, etc., the formation rate is calculated as

$$
\text { Rate }_{\mathrm{DNS}}=f_{\mathrm{b}} \frac{N_{\mathrm{obs}}}{T_{\mathrm{PSR}}},
$$

where $T_{\mathrm{PSR}}$ is the observable lifetime of the pulsar and all other uncertainties have been represented by a single factor, $f_{\mathrm{b}}$, whose value may be uncertain by 1-2 orders of magnitude. With best guesses for $f_{\mathrm{b}}$, the current rate inferred for our Galaxy from the observations fall in the range: $\sim 10^{-6}-10^{-5} \mathrm{yr}^{-1}\left(\mathrm{Phinney}^{-6}\right.$ 1991; Narayan, Piran, \& Shemi 1991; Curran \& Lorimer 1995; van den Heuvel \& Lorimer 1996). Bailes (1996) estimated an upper limit for the formation rate of these systems using the fact that all of the observed pulsars in DNS systems are recycled and none of the companion neutron stars are observed as normal pulsars. Although normal pulsars have much shorter lifetimes, they dominate the observed pulsar population because they do not require binary accretion to "recycle" them. The formation rate of single pulsars is roughly $\sim 0.008 \mathrm{yr}^{-1}$ and, at the time of the calculation by Bailes (1996), roughly 650 normal radio pulsars were observed. Using eq. (11), we can then estimate the value of $f_{\mathrm{b}} / T_{\mathrm{PSR}}$. If we assume that the collapse and pulsar formation of the secondary is not too dissimilar from that of single stars (since the core of a massive star is not affected greatly by its binary companion, this assumption may not be too bad), then we can use the value of $f_{\mathrm{b}} / T_{\mathrm{PSR}}$ derived from the pulsar population to place an upper limit on the DNS formation rate. Since the number of normal secondary pulsars is $0(<1)$, the current DNS formation rate in our Galaxy is then (Bailes 1996)

$$
\text { Rate }_{\mathrm{DNS}}<\left(\frac{f_{\mathrm{b}}}{T_{\mathrm{PSR}}}\right)_{\mathrm{PSR}}=10^{-5} \mathrm{yr}^{-1} .
$$

Since this calculation, the number of observed pulsars, the number of DNS systems has increased, and the pulsar lifetime has changed, and this upper limit has risen to $10^{-4} \mathrm{yr}^{-1}$ (Arzoumanian, Cordes, \& Wasserman 1999).

\section{2. $\quad$ Black Hole + Neutron Star Binaries}

As BH/NS binaries merge, the neutron star is tidally disrupted. Some of the material accretes directly, producing little emission, but the remainder forms an accretion disk of a few tenths of solar mass which can power a GRB (Lee \& Kluzniak 1995; Eberl 1998; Eberl et al. 1999). Like the DNS mergers, if the disk is hot and viscous, the relativistic outflows created by $\mathrm{BH} / \mathrm{NS}$ mergers are probably of short duration, but somewhat more energetic than their DNS counterparts because of the larger mass reservoir.

Merging BH/NS binaries are formed by pathways that are similar to DNS binaries. In general, the collapse of a massive star can form black holes in two distinct ways (Woosley \& Weaver 1995; Fryer 1999). The star can explode, but with such a weak shock that much of the stellar mantle of heavy elements falls back onto the neutron star, causing it to further collapse into a black hole. Alternatively, especially for the more massive stars, the ram pressure of the infalling stellar material may be too large to allow the shock to propagate at all and the star may collapse directly into a black hole. The two limits for black hole

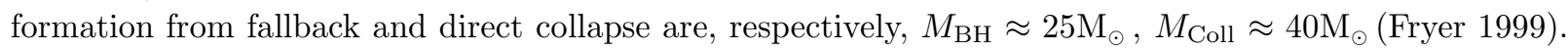
The "standard" formation scenario for BH/NS systems (Scenario IV, Fig. 5) begins with two massive stars, 
the primary having a mass greater than $M_{\mathrm{BH}}$. As with the standard DNS formation scenario, the primary evolves off the main sequence, overfills its Roche-lobe, and transfers mass to its companion. The primary continues to evolve to the end of its life, forming a black hole and, possibly, a supernova explosion. With the exception of extremely large kicks, the system remains bound, creating a binary consisting of a black

hole and a massive star. This system passes through an X-ray binary phase (e.g., Cyg X-1, LMC X-1) and then evolves through a common envelope as the secondary star expands. During this common envelope phase (in the standard model) the black hole spirals into the massive secondary and ejects the secondary's hydrogen envelope. After the secondary's supernova explosion, a BH/NS binary forms which then merges via gravitational wave emission.

$\mathrm{BH} / \mathrm{NS}$ binaries can also form from neutron star binaries in which the neutron star of the primary gains too much matter during common envelope phase and collapses into a black hole ( $\$ 2.1$, Scenario V, Fig. 6). This formation scenario produces binaries consisting of a low-mass black hole $\left(\sim 3 M_{\odot}\right)$ and a neutron star which could generate the most energetic BH/NS GRBs (Eberl 1998, Eberl et al. 1999).

Finally, just as with the DNS systems, a BH/NS binary can form under a third mechanism avoiding any common envelope evolution for appropriate kick magnitudes and directions (Scenario VI, Fig. 7). Like the DNS kick formation scenario, the kick scenario is probably not important for BH/NS formation.

Although BH/NS binaries have not yet been observed, their formation rate is probably comparable or even larger than that of DNS systems. As with the double neutron stars, we can determine an observational upper limit to the $\mathrm{BH} / \mathrm{NS}$ rate in the Galaxy (assuming the observational biases are similar to those for single pulsars) of $\sim 10^{-4} \mathrm{yr}^{-1}$.

\subsection{Black Hole - White Dwarf Binaries}

As WD/BH binaries merge, the white dwarf is tidally disrupted and most of its matter is converted into an accretion disk around the black hole. The accretion of this disk material onto the black hole can drive a gamma-ray burst (Fryer et al. 1999). Although the accretion disk is more massive than that formed in DNS mergers, its radius is much larger so the accretion time scale is longer and neutrino energy transport less efficient. However, the black hole is rapidly rotating and up to $10^{53}$ erg of either rotational energy or disk binding energy is potentially available on a viscous time scale for the disk. MHD powered jets might provide long-duration GRBs.

Not surprisingly, there is also more than one way to produce a binary containing a white dwarf and a black hole. Our first scenario begins with a binary consisting of a primary with mass greater than $M_{\mathrm{BH}}$ and a low-mass $\left(<10 \mathrm{M}_{\odot}\right)$ companion (Scenario VII, Fig. 8). After the primary collapses to form a black hole, one has a binary consisting of a black hole and a main sequence star. If the mass of the main sequence companion is less than $\sim 2-3 M_{\odot}$, magnetic braking will drive the binary together, forming a low-mass X-ray binary (LMXB). More massive companions will not become X-ray binaries until they evolve off the main sequence (e.g. LMC X-3, 2023+338 Nova Cyg, J0422+32 Nova Per). For masses of $5-10 \mathrm{M}_{\odot}$, a common envelope phase is likely to occur which decreases the orbital separation. These stars eventually evolve into high mass white dwarfs, a fraction of which will merge with the black hole primary in a Hubble time.

Alternatively, these systems may form from neutron star binaries with low-mass $\left(<10 M_{\odot}\right)$ companions (Scenario VIII, Fig. 9). If the neutron star enters a common envelope phase when the low-mass companion 
expands into a giant, it can accrete too much material and collapse to a black hole. BH/WD binaries may also form avoiding the first common envelope phase (where the low-mass companion spirals into the massive primary) entirely with a "well-placed" kick (Scenario IX, Fig. 10). Just as with Scenario VII, the system evolves briefly through an X-ray binary phase and possible common envelope phase which tightens the orbit.

Just as with $\mathrm{BH} / \mathrm{NS}$ binaries, there are no observed closely interacting $\mathrm{BH} / \mathrm{WD}$ binaries and our estimates must be very uncertain. The lack of a detectable system so far implies only

$$
\text { Rate }_{\mathrm{WD} / \mathrm{BH}} \lesssim \frac{V_{\text {Galaxy }}}{V_{\mathrm{WD} \mathrm{Obs}}} T_{\mathrm{WD}}^{-1} \approx \frac{(10 \mathrm{kpc})^{2}(1 \mathrm{kpc})}{(10 \mathrm{pc})^{3}} \times\left(10^{10} \mathrm{yr}\right)^{-1} \approx 0.01 \mathrm{yr}^{-1} .
$$

In this paper, we will show in fact that the predicted rate is $\sim 5$ orders of magnitude less than this upper limit.

\subsection{Collapsars}

The only BHAD GRB progenitor which does not necessarily require a binary system (though, as we shall see, being in a binary still helps) is the collapsar (Woosley 1993). Collapsars are formed when the collapse of the iron core of a rotating massive supernova progenitor proceeds directly to a black hole. As the stellar mantle falls into the newly formed black hole, angular momentum slows the collapse along the equator, ultimately forming an accretion disk which powers the GRB (Woosley 1993, MacFadyen \& Woosley 1999). Collapsars can produce long duration bursts ( $\gtrsim 10 \mathrm{~s}$ ) with a large range of burst energies by either neutrino or MHD processes. In order that the jet not dissipate its energy in the hydrogen envelope of a red supergiant star, it is necessary that the collapsar occur in a hydrogen stripped, Wolf-Rayet star.

The collapsar model requires that no supernova shock is launched, and hence collapsars form only from stars whose masses exceed $M_{\text {Coll }}$. One obvious consequence is that GRBs from this model would closely trace star formation. There will also be significant amounts of circum-burster material from the pre-SN stellar wind and the gas in the surrounding star forming medium. The afterglow (and even some bursts) would be produced by shock interactions with this wind, not with the ordinary interstellar medium. Scenario X (Fig. 11) is the simplest possibility for producing a collapsar. The iron core of a single very massive star collapses promptly to a black hole.

Current simulations of collapsars (MacFadyen \& Woosley 1999) suggest that a successful collapsar model requires specific angular momenta of the massive star cores to be in the range: $j \approx 3 \times 10^{16}-2 \times 10^{17}$

and that the massive star has lost most of its hydrogen envelope, but retains a sufficiently large helium core to collapse and power a GRB. If the angular momentum is below this range, an accretion disk does not form and the material falls more or less directly into the black hole. If the angular momentum is too large, the disk does not efficiently dissipate energy through neutrino emission and a weak outflow instead of an accretion disk results. The requisite high mass of the helium star may be more easily attained in a star of low metallicity (MacFadyen \& Woosley 1999).

The biggest uncertainty in estimating the rate of collapsar models, however, is the angular momentum. There are diverse views regarding how much angular momentum is retained in the stellar cores of massive stars when they die. Heger (1998) modeled the evolution of rotating massive stars and found that the cores of most stars naturally have angular momenta close to the range required by MacFadyen \& Woosley (1999). However, Spruit \& Phinney (1998) have argued that magnetic fields will couple the cores of stars to their 
envelopes, causing the star to rigidly rotate. As the star became a red supergiant (or even a blue one), the core rotation would essentially halt. No GRB could be produced if this occurred. However, the results of Spruit \& Phinney rest upon uncertain assumptions involving the evolution of magnetic field instabilities, especially those responsible for producing a radial component in the region where the core and envelope are interacting. Here we will assume that there is no such magnetic coupling and all massive stars are rotating within the range required by MacFadyen \& Woosley (1999). It will turn out however that, because of the stellar mass loss prescription we adopt, massive stars other than those interacting binaries, will produce a minority of collapsar progenitors. If the stellar envelope is removed very early by a companion star, perhaps the rotation of the core is not slowed as much. However, one may want to multiply our final numbers for collapsar rates by an uncertain factor less than one to account for inadequate angular momentum.

Binary companions can also remove hydrogen envelopes during a common envelope phase. After common envelope evolution, which uncovers the helium core, the primary star (if massive enough) collapses into a black hole. If the angular momentum of the primary is appropriate, a collapsar is formed (Scenario XI, Fig. 12). Note that this system may remain in a binary and later evolve into a $\mathrm{BH} / \mathrm{NS}$ or $\mathrm{WD} / \mathrm{BH}$ binary.

Even if both Scenarios X and XI fail (e.g. because of magnetic braking of the core's rotation; Spruit \& Phinney 1998), it is still possible to form a collapsar in a binary in which the two stars are of comparable mass, and evolve off the main sequence together. If the two helium cores merge during their common envelope evolution, they will form a massive helium core with a large amount of angular momentum even if their individual cores were rotating slowly (Scenario XII, Fig. 13). Scenario XII by itself then might be thought of as giving a lower bound to the collapsar rate.

\subsection{Helium Star Mergers}

The helium star merger model (Fryer \& Woosley 1998) is another outcome possible for the common envelope phase so central to making DNS and BH/NS binaries. If the inspiralling compact object does not have sufficient orbital energy to eject its companion's hydrogen envelope, it moves on into the helium core (e.g., in Scenarios I, IV, V; see Figs. 2,5,6). As the helium core and compact object merge, the Bondi-Hoyle accretion rate may become extremely high $\left(1 M_{\odot} \mathrm{s}^{-1}\right)$ and the compact object quickly accretes enough to become a black hole if it has not already. The angular momentum of the helium core/black hole binary is injected into the helium core, forming a massive disk $\left(\sim 4 M_{\odot}\right)$ around a spinning black hole (Scenario $\mathrm{XIV}$ ). This BHAD GRB model has not been simulated in detail, but it is likely to produce bursts of long duration similar to that of the collapsar model, though probably longer. Unlike the collapsar model, there is no concern about having enough angular momentum. Indeed the problem may be too much of it.

Alternatively, the merging process can be initiated by a kick that places the neutron star inside its companion, ultimately leading to the merger of the neutron star with its companion's helium core, again forming an accretion disk around a black hole. However, the odds of such a well-placed kick are again slim, and this scenario never occurred in our simulations and hence we do not list it separately. 


\section{The Population Synthesis Calculation}

For our numerical calculations, we used a modified version of the Monte Carlo population synthesis code of Fryer, Burrows, \& Benz (1998). This code has previously been tested against, and, within the errors, agrees with the low-mass X-ray binary formation calculations of Kalogera \& Webbink (1998) and the high-mass X-ray binary formation simulations of Dalton \& Sarazin (1995). Calculations of compact object formation begin with a set of initial binary conditions, each of which brings some element of uncertainty to the result: the initial mass function, the fraction of binary stars, the binary mass ratio distribution, the distribution of orbital separations, and initial eccentricities. Monte-Carlo calculations then evolve the binary stars and algorithms for both the single-star evolution (e.g., He core mass, radii during giant phase, winds) and the binary-star evolution (e.g., mass transfer, common envelope evolution) must also be assumed. Finally, one must make some approximations to the supernova explosion mechanism and its results - especially neutron star (and black hole) kicks. We allow all these parameters to range over a reasonable range of uncertainties, applying constraints where we can, to derive a range in resultant GRB rate. The effects of the free parameters are demonstrated in Tables 2-7. An estimated range of rates, including all the uncertainties, is given Table 8 .

With such a large number of free parameters, we cannot survey the entire parameter space, so we instead chose a "standard" model from which to examine the effects of each individually. Our standard model uses the following assumptions (to be described in greater detail in subsequent subsections): $\alpha_{\mathrm{IMF}}=2.7$, circular initial orbits, a logarithmic distribution of initial separations $[P(A) \propto 1 / A]$, a flat probability distribution for the mass ratio, a binary fraction of $40 \%$, a supernova rate of $0.02 \mathrm{yr}^{-1}$, isotropic supernova kicks given to both neutron stars and black holes, a lower mass limit for black hole formation of $25 M_{\odot}$, stellar radii for mass-transfer and common-envelope evolution set to $1 / 4$ that given by Kalogera \& Webbink (1998), helium star masses as given by Kalogera \& Webbink (1998) but with wind mass-loss rates set to $10 \%$ of those used by Woosley, Langer, \& Weaver (1995), mass transfer parameters $\left(\alpha_{\mathrm{MT}}, \beta_{\mathrm{MT}}\right)$ of 1.,0.8 respectively, and common envelope efficiency, $\left(\alpha_{\mathrm{CE}}\right)$, equal to 0.5. For other parameters not discussed in this section, we used the standard values given in Fryer, Burrows, \& Benz (1998). Unless otherwise stated, each Monte-Carlo simulation models 20 million massive binaries and the plots and tables include only those binaries which will merge within a Hubble time. With 20 million binaries, a supernova rate of $0.02 \mathrm{yr}^{-1}$ and a binary fraction of $40 \%$, a binary merger rate of $1 \mathrm{Myr}^{-1}$ corresponds to 5000 systems and statistical errors of roughly $2 \%$.

How calculations normalized to the supernova rate in our Galaxy are translated into universal rates based upon a history of star formation is discussed in $\S 4.2$.

\subsection{Initial Conditions}

The initial conditions in our population synthesis calculations include distributions of the zero-age main-sequence masses, of orbital separations, and of orbital eccentricities for the binaries. The primary mass is determined using an initial mass function (IMF):

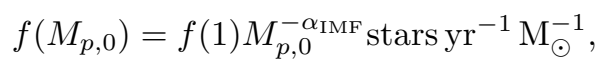

where $M_{p, 0}$ is the initial mass of the primary, and $\alpha_{\mathrm{IMF}}$ is typically set to 2.7 (Scalo 1986). Rather than fix $f(1)$, we fix the supernova rate $(\S 3.2)$. Since we are only interested in compact binaries, we require that the primaries have a mass of at least $10 M_{\odot}$. We allow massive stars up to $150 M_{\odot}$ to be formed, but our choice 
of the initial mass function severely restricts the role these massive stars play in our population synthesis. Repeating the calculation with $\alpha_{\mathrm{IMF}}=2.35$ yields similar (within a factor of 3 ) merger rates (Table 2).

Secondary masses are much more difficult to determine. The standard technique (see Hogeveen for a review 1990) prescribes a mass ratio $\left(q=\frac{M_{s}}{M_{p}}\right)$ distribution $P(q)$ by

$$
P(q) \propto q^{-\alpha_{\mathrm{MR}}} .
$$

Because it is extremely difficult to detect low-mass companions of massive stars, the value of $\alpha_{\mathrm{MR}}$ ranges from -1 or 0 (Garmany, Conti, \& Massey 1980) to 2.7 for $q>0.35$ and 0 . for $q<0.35$ (Hogeveen 1990). The discrepancy in these mass ratios arises not from different data, but a different interpretation of the data. Of the 67 massive stars measured by Garmany, Conti, \& Massey (1980), 30\% contained close binaries with mass ratios exceeding 0.4 , and, according to their analysis, $40 \%$ of massive stars were in binaries. Hogeveen (1990), on the other hand, argues that Garmany, Conti, \& Massey (1980) underestimated the number of binaries with low-mass companions and instead estimates a much larger fraction of binary massive stars $(\sim 70 \%)$ peaked toward extreme mass ratios $\left(\alpha_{\mathrm{MR}}=2.7\right)$. For the merger rates in Table 2, we always assume a binary fraction of $40 \%$. However it is likely that the binary fraction, and hence the merger rates, for the $\alpha_{\mathrm{MR}}=2.7$ calculations are a factor of 2 higher. Even with this increase of 2 of the binary fraction, these extreme mass ratios predict over an order of magnitude fewer DNS binaries (which, dominated by Scenario II, require nearly equal mass binaries) and nearly a factor of 3 more WD/BH binaries (Table 2). In this case, $\mathrm{WD} / \mathrm{BH}$ mergers are more common than DNS mergers!

For initial orbital separations, we use the distribution introduced by Kraicheva et al. (1979):

$$
P\left(A_{0}\right) \propto 1 / A_{0}
$$

with constraints on the innermost initial separation (equal to 3 times the sum of the stellar radii) and on the outermost initial separation (equal to $10^{4} R_{\odot}$ ). Increasing this inner separation by a factor of 2 , or increasing the outer separation by 2 orders of magnitude, raises (or lowers for the outer limit) the merger rates by less than 20-30\%. Likewise, assuming the initial eccentricity is evenly distributed between 0 and 0.9 (versus assuming initially circular orbits) also has less than a $40 \%$ effect (Table 2).

With the exception of the mass ratio distribution, uncertainties in the initial conditions have little effect on the merger rates of neutron star binaries. For our standard set of parameters, we use, as do most of the previous calculations of DNS binaries, a flat mass ratio distribution $\left(\alpha_{\mathrm{MR}}=0\right)$ with a binary mass fraction of $40 \%$. However, the standard formation scenario for low-mass X-ray binaries requires that the mass ratio distribution is peaked toward extreme mass ratios (Kalogera \& Webbink 1998). Unless low-mass X-ray binaries are formed via some alternative mechanism, $\alpha_{\mathrm{MR}}$ must be higher $(\sim 2.7)$, and we are overestimating the DNS merger rate by over an order of magnitude and underestimating the WD/BH merger rate by a factor of 3. For the rest of our standard set of parameters, we use the Scalo (1986) initial mass function, initially circular orbits, and the distribution of separations and inner and outer limits discussed in the previous paragraph.

\subsection{Uncertainties in the Supernova Model}

To form the binaries containing compact objects that might eventually produce BHAD GRBs, we must not only follow the evolution of binary stars, but also take into account the many uncertainties associated with the supernova process. One key quantity, to which all our formation scenarios are proportional, is the 
supernova rate and its history in the universe. We assume a current Galactic supernova rate of $0.02 \mathrm{yr}^{-1}$, consistent with the results of Cappellaro et al. (1997), but even that is uncertain by at least a factor of 2 . As one moves to the distant past, the rate is presumably larger for our Galaxy, but then galaxies too were evolving in size and luminosity. We discuss in $\S 4$ the scaling of our supernova rate to distant redshifts.

Another source of great uncertainty in estimating the formation rate of DNS and BH/NS systems is an inadequate understanding of the kicks imparted to neutron stars (or black holes) at birth. Growing evidence suggests that neutron stars receive a momentum boost, or "kick", $\left(v_{\text {kick }}^{\text {mean }} \sim 300-500 \mathrm{~km} \mathrm{~s}^{-1}\right)$ during their formation, probably due to some asymmetry in the explosion itself. Evidence includes the pulsar velocity distribution (Dewey \& Cordes 1987; Lyne \& Lorimer 1994; Fryer, Burrows, Benz 1998; Cordes \& Chernoff 1998), associations of neutron stars with supernova remnants (Caraveo 1993; Frail, Goss \& Whiteoak 1994 although see Gaensler \& Johnston 1995), observations of bow shocks produced by neutron stars as they plow through the interstellar medium (Cordes, Romani, \& Lundgren 1993), and the formation scenarios for DNS systems and many other close binary systems(DNS systems: Flannery \& van den Heuvel 1975; Burrows \& Woosley 1986; Yamaoka, Shigeyama, \& Nomoto 1993; Fryer \& Kalogera 1997; spin/orbit angles in binaries: Kaspi et al. 1996; Wasserman,Cordes, \& Chernoff 1996; eccentric binaries: van den Heuvel \& Rappaport 1986). Although small kicks $\left(\sim 200 \mathrm{~km} \mathrm{~s}^{-1}\right)$ are required to match the observed DNS systems, larger kicks tend to disrupt the binaries. Recent analyses of the pulsar velocity distribution suggests a bimodal kick distribution with roughly half of the neutron stars receiving kicks smaller than $200 \mathrm{~km} \mathrm{~s}^{-1}$ and the other half receiving kicks greater than $500-600 \mathrm{~km} \mathrm{~s}^{-1}$ (Fryer, Burrows, \& Benz 1998). The binary systems whose neutron stars receive very large kicks become unbound. The remaining systems may form DNS and BH/NS binaries. The choice of kick magnitude has a strong effect on the formation rate of these two compact binary progenitors (Fig. 14).

During the supernova explosion, the binary only remains bound only if $M_{\text {ejecta }}<0.5 M_{\text {sys }} r / a_{0}$ where $M_{\text {sys }}, r$, and $a_{0}$ are the total mass, orbital separation, and semi-major axis of the binary (Hills 1983). With the circular orbits important for close-binary formation (common envelope evolution circularizes the orbits), this equation becomes $M_{\text {ejecta }}<0.5 M_{\text {sys }}$. When a kick is imparted onto the neutron star during the supernova explosion, the criterion for bound systems is:

$$
M_{\text {ejecta }}<0.5 M_{\text {sys }}\left[1-2\left(v_{\text {kick }} / v_{\text {orb }}\right) \cos \theta \cos \phi-\left(v_{\text {kick }} / v_{\text {orb }}\right)^{2}\right],
$$

where $v_{\text {kick }}$ and $v_{\text {orb }}$ are the magnitudes of the kick and orbital velocities respectively. $\theta$ and $\phi$ are the polar angle directions of the kick velocity with respect the the orbital velocity. Although for some systems, a kick might actually prevent the disruption of the binary (this is important for low-mass X-ray binary formation: Kalogera \& Webbink 1998; Kalogera 1998, Fryer, Burrows, \& Benz 1998), in general, the kick tends to unbind binaries. For an isotropic kick distribution, the probability that a system remains bound after the supernova explosion is given by (Brandt \& Podsiadlowski 1995):

$$
P\left(v_{\text {kick }}, v_{\text {orb }}, M_{\text {ejecta }}, M_{\text {sys }}\right)=0.5+0.5 \frac{v_{\text {orb }}}{v_{\text {kick }}}\left[\frac{M_{\text {sys }}-M_{\text {ejecta }}}{M_{\text {sys }}}-0.5\left(\frac{v_{\text {kick }}}{v_{\text {orb }}}\right)^{2}-0.5\right] \text {. }
$$

We performed a series of Monte-Carlo simulations assuming delta function kicks (Fig. 14). Note that the merger rates of DNS binaries initially increase as the mean kick velocities increase from $0 \mathrm{~km} \mathrm{~s}^{-1}$. The actual binary formation rates are much higher for lower kicks (Fig. 15), but higher kicks are required to form the close binaries which will merge within a Hubble time. The presupernova orbital separation must be sufficiently wide to avoid a common envelope phase which would destroy the binary and a kick is required to tighten the orbits and make close merging binaries. In DNS binary formation, the primary's 
neutron star must avoid common envelope evolution with its secondary's helium star (Fryer \& Kalogera 1997) or it will accrete too much material and become a BH/NS GRB. Similarly, low-mass stars must have sufficiently wide orbital separations so that they do not merge with their massive companions before the massive star collapses to form a black hole. Clearly, the radius of the helium star is important for these calculations. We will return to this effect in $\$ 3.3$.

The actual kick distribution is probably not a delta function, but spans wide range of velocities. In Table 3, we give the merger rates for both a Maxwellian velocity distribution (with a mean of $450 \mathrm{~km} \mathrm{~s}^{-1}$ ) and a double peaked distribution similar to that described by Fryer, Burrows, \& Benz (1998) with two peaks near $100 \mathrm{~km} \mathrm{~s}^{-1}$ and $600 \mathrm{~km} \mathrm{~s}^{-1}$ with $\sigma=50,150 \mathrm{~km} \mathrm{~s}^{-1}$ respectively ("FBB kick distribution"). Note that although the FBB and Maxwellian kick distributions have roughly the same average magnitude (and both roughly explain the pulsar velocity distribution), the FBB kick distribution produces many more binary systems. Because the merger rates depend so sensitively upon the kick distribution, we will employ several kick velocities in our studies.

At the present time, no known mechanism provides the large kicks implied by the pulsar observations. It is therefore doubly difficult to predict the distribution for black holes since the velocities of single black holes have not been measured. Kick mechanisms driven by asymmetries in the supernova explosion seem likely to impart smaller kick velocities to black holes, if only because they are more massive. If anisotropic neutrino emission is involved, for example, the black hole may also form before much neutrino momentum has been emitted and therefore experience less recoil. As one might expect, setting the black hole kick magnitudes to $10 \%$ that of neutron stars implies much larger black hole binary merger rates (Table 3 ).

Another uncertainty in supernova explosions is the limiting main sequence mass star that will form a black hole, either by fallback $\left(M_{\mathrm{BH}}\right)$ or prompt collapse $\left(M_{\text {Coll }}\right)$. Current supernova models (Fryer 1999) suggest that $M_{\mathrm{BH}}$ is not much above $25 \mathrm{M}_{\odot}$ which agrees roughly with observations (Maeder 1992; Kobulnicky \& Skillman 1997; Portegies Zwart, Verbunt, \& Ergma 1992; Ergma \& van den Heuvel 1998). In Fig. 16 (and Table 3), we show results from simulations with $M_{\mathrm{BH}}$ ranging from $25 M_{\odot}$ to $85 M_{\odot}$ and $M_{\mathrm{Coll}}=M_{\mathrm{BH}}+15 \mathrm{M}_{\odot}$ using the FBB kick distribution. A higher mass limit leads to the formation of fewer black holes, and hence, fewer BH/WD binaries and collapsars. The merger rate of BH/NS binaries does not depend sensitively on the black hole mass limit. This is because the dominant formation scenario (Sc. V) requires the primary to collapse into a neutron star which then accretes matter in a common envelope phase and collapses to a black hole (see \$3.4). If hyper-critical accretion does not occur, formation scenario $\mathrm{V}$ does not work and, as shown in Fig. 16, the formation rate of BH/NS binaries depends more sensitively upon the critical black hole mass limit. For our standard set of simulations, we set $M_{\mathrm{BH}}=25 \mathrm{M}_{\odot}$ and $M_{\mathrm{Coll}}=40 \mathrm{M}_{\odot}$.

In our simulations, we also assume that massive stars collapse to form black holes with masses set to $1 / 3$ the primary star's mass at the time of collapse. For stars with masses between $M_{\mathrm{BH}}$ and $M_{\mathrm{Coll}}$, a supernova ejects some of the mass. Even beyond $M_{\text {Coll }}$, not all of the star will collapse to form a black hole. As the gamma-ray burst forms, it also may drive off part of the helium core. The resultant mass distribution of black holes in binaries with massive companions is double peaked (Fig. 17). The large peak at $2.4 \mathrm{M}_{\odot}(>50 \%$ of the black holes $)$ is caused by those black holes formed by hyper-critical accretion onto neutron stars in common envelope evolution. These low-mass black holes will produce the most energetic BH/NS merger GRBs. Their actual masses depend sensitively upon common envelope evolution, but will probably not exceed $4 \mathrm{M}_{\odot}$. The second, spread-out distribution, is even more difficult to quantify, as it depends not only upon binary evolution, but also the rates of stellar winds and the mass of the ejecta in supernova explosions. In fact, assuming that the mass of the black hole is only $1 / 3$ that of the primary star's mass is probably a lower limit to the black hole mass (Fryer 1999). Setting the black hole mass equal 
to the collapsing star's mass slightly increases the rates of black hole binaries for low kicks. However, for high kicks, the high momentum imparted to these more massive systems tends to unbind the system.

\subsection{Stellar Evolution}

Uncertainties in stellar models are often neglected but, as we shall show in this section, they can have a large effect on the rates for the BHAD models. The most important uncertainty in the stellar models is the stellar radius, especially as the star moves off the main sequence. The radius of a star is important because it determines if, and when, a binary system passes through a common envelope or mass transfer phase. Accurate stellar radii are difficult to determine observationally and current theoretical models concentrate on the cores of massive stars. Many of the theoretically predicted radii depend upon artificial outer boundary conditions. Indeed, especially for massive giant stars and for Wolf Rayet stars, the radius is not a very precisely defined quantity. Observations tell us only about the radius of the photosphere. This size can be much larger than the radius which is important for population synthesis calculations, that is, the radius where the stellar density is sufficient to drive common envelope evolution (see \$3.4).

For both giant and helium stars, our standard model assumes that the common envelope radius is $25 \%$ the radius given in the stellar model fits by Kalogera \& Webbink (1998). If we raise this to the values given by Kalogera \& Webbink (1998), both the rates of DNS and BH/NS binaries decrease over an order of magnitude in some cases (Table 4). Increasing stellar radii causes more binaries to go into common envelope phases, but, more importantly, increasing helium-star radii cause more binaries to merge (producing fewer compact binaries and more helium merger GRBs).

In addition, many of the formation scenarios (e.g. I, II, IV, V) produce compact stars (neutron star, black hole) around helium-star companions. As the helium-star radius increases, the orbital separations of compact binaries become wider and consequently fewer merging compact binaries are produced. Thus, for a given kick distribution, increasing the helium-star radius causes both the number of binaries and the fraction of those binaries which will merge in a Hubble time to decrease (see Fryer \& Kalogera 1997).

Winds affect both helium star masses and radii. For instance, if we use a mass loss rate like that of Woosley, Langer, \& Weaver $(1993,1995)$, helium masses are relatively small (e.g., a massive star forming a $20 M_{\odot}$ helium core will end its life with a $3.5 M_{\odot}$ helium core), and their radii are greater. For larger helium star radii, fewer close binaries form, and the merger rates of both DNS systems and BH/NS systems decrease (Table 4). In addition, high winds raise the critical mass for black hole formation (less massive stars simply do not have massive enough cores at time of collapse to prevent a supernova explosion) which reduces the rates of all black hole systems (the collapsar rate decreases by an order of magnitude). Note that this mass loss is an extreme case and some helium stars must not have such drastic winds if the formation scenarios for black hole X-ray binaries such as V404 Cyg, Cyg X-1, and XN Mus 92, are correct (White \& Paradijs 1996). In our simulations, we parameterize the mass loss rate as a fraction of the rate used by Woosley, Langer, \& Weaver $(1993,1995): \alpha_{\text {Wind }}=($ Mass Loss) $/$ (Mass LosswlW $)$. Current mass loss estimates suggest that the WLW models overestimate the maximum mass-loss by a factor of two to three. The effect of mass-loss is minimal unless $\alpha_{\text {Wind }}$ exceeds 0.6 (Fig. 18). If the mass loss never exceeds this value, only the collapsar model is affected at all by mass loss. As the mass loss rises from $\alpha_{\text {Wind }}=0$. to $\alpha_{\text {Wind }}=0.6$, the collapsar rate is cut by a factor of $2-3$.

We have simplified the uncertainties by assuming the error in the red supergiant wind is the same as that of Wolf-Rayet winds. We decrease the mass loss in the red supergiant wind by the same factor that 
we decrease the mass loss for helium stars by lumping the mass loss uncertainty into one parameter. With this assumption, we are probably underestimating the number of collapsars formed via winds (Sc. X), perhaps grossly so. Using just one parameter, those stars with winds strong enough to uncover the helium core also lose too much of their helium core to collapse directly into a black hole. Observations suggest that single stars above $\left(\sim 40 \mathrm{M}_{\odot}\right)$ must lose their hydrogen envelope (Chiosi \& Maeder 1986), which would require a high value for the wind parameter: $\alpha_{\text {Wind }}>0.8$. However, to match the black hole binaries, the wind parameter for helium stars must be low: $\alpha_{\text {Wind }}<0.5$ (Kalogera 1999). Assuming that black hole binaries are formed in systems which do not go into common envelope evolution until after central helium exhaustion, Wellstein \& Langer (1999) are able to explain the black hole binaries but maintain a high mass

loss rate. If their models are correct, very few massive stars, single or binary, would have enough mass prior to collapse to form black holes directly, and the formation rate of collapsars in the present-day universe would drop dramatically. However, since mass loss is metal dependent, the collapsar rate might be larger at higher redshifts than it is today (MacFadyen \& Woosley 1999).

\subsection{Binary Evolution}

For binaries to merge and form GRBs, the progenitor systems must have short periods. consequently, most will undergo some sort of active binary evolution. We have assumed that if the mass of the expanding star $\left(M_{\exp }\right)$ is less than $\sim 2.5$ times the mass of its companion $\left(M_{\text {comp }}\right)$, the companion is unable to incorporate more than a small fraction of the overflowing mass (Webbink 1979). A fraction $\left(\beta_{\mathrm{MT}}\right)$ of the expanding star's envelope is accreted by the companion, and the remainder is lost from the system, carrying away a specific angular momentum $\left(\alpha_{\mathrm{MT}}\right)$. The orbital separation of the system $(A)$ in terms of its initial separation $\left(A_{0}\right)$ after mass transfer is given by (Podsiadlowski, Joss, \& Hsu 1992):

$$
\frac{A}{A^{0}}=\frac{M_{\text {exp }}+M_{\text {comp }}}{M_{\exp }^{0}+M_{\text {comp }}^{0}}\left(\frac{M_{\text {exp }}}{M_{\text {exp }}^{0}}\right)^{C_{1}}\left(\frac{M_{\text {comp }}}{M_{\text {comp }}^{0}}\right)^{C_{2}}
$$

where the values of the constants are:

$$
\begin{gathered}
C_{1} \equiv 2 \alpha_{\mathrm{MT}}\left(1-\beta_{\mathrm{MT}}\right)-2 \\
C_{2} \equiv \frac{-2 \alpha_{\mathrm{MT}}}{\beta_{\mathrm{MT}}}\left(1-\beta_{\mathrm{MT}}\right)-2
\end{gathered}
$$

and

$$
M_{\mathrm{comp}}=\beta_{\mathrm{MT}}\left(M_{\exp }^{0}-M_{\mathrm{exp}}\right)+M_{\mathrm{comp}}^{0},
$$

and where the superscript 0 denotes pre-mass transfer phase values. Although the values of $\alpha_{\mathrm{MT}}$ and $\beta_{\mathrm{MT}}$ are relatively uncertain, varying $\alpha_{\mathrm{MT}}$ from $0.5-2.0$ does not change the GRB rates by more than $30 \%$ (Table 5). However, changing the amount of material lost by the system during mass transfer has a similar effect to that of changing the mass loss by winds. Increasing $\beta_{\mathrm{MT}}$ from 0.5 to 1.0 decreases the $\mathrm{BH} / \mathrm{NS}$ merger, collapsar, and helium merger rates by up to an order of magnitude (Table 5).

If $M_{\exp } \gtrsim 2.5 M_{\text {comp }}$ (if the companion is a compact object, this factor of 2.5 reduces to 1.0 ), the companion can not adjust to the accreting mass and instead enters into a common envelope phase. Once a common envelope is created, the orbital separation decreases rapidly, probably in $1-1000 \mathrm{yr}$ (see Sandquist et al. 1998 and references therein). This inspiral continues until the binary is able to shed the expanding 
star's hydrogen envelope leaving a bare helium core. One can estimate the final orbital separation $\left(A_{f}\right)$ in terms of the initial separation $\left(A_{i}\right)$ of the binary after common envelope evolution (Webbink 1984):

$$
\frac{A_{f}}{A_{i}}=\frac{\alpha_{\mathrm{CE}} r_{L} q}{2}\left(\frac{M_{H e}}{M_{s}-M_{H e}+\frac{1}{2} \alpha_{\mathrm{CE}} r_{L} M_{N S}}\right)
$$

where $M_{s}, M_{H e}$, and $M_{N S}$ are, respectively, the masses of the secondary, the secondary's helium core, and of the neutron star and $r_{L}=R_{L} / A_{i}$ is the dimensionless Roche lobe radius of the secondary. $\alpha_{\mathrm{CE}}$ is a parameter designed to represent how efficiently the orbital energy ejects the hydrogen envelope and includes assumptions about the binding energy of the expanding star and the amount of orbital energy injected, per gram, into the hydrogen envelope. The value of $\alpha_{\mathrm{CE}}$ is not well constrained, nor is it constant. It depends both upon the mass and evolutionary stage of the expanding star when a common envelope phase occurs. Varying the common envelope efficiency over a range (0.25-1.0) varies the GRB rates by less than a factor of 3 (Table 6), and one might naively assume that common envelope evolution does not affect the GRB rates.

However, common envelope evolution may be drastically different from that given by the simple $\alpha_{\mathrm{CE}}$ prescription. Nearly all past population synthesis calculations ignore the possible merger of an inspiralling compact object with the core of its companion in a common envelope phase. In most cases, previous population synthesis codes simply did not check to see when the merging compact object fell within the helium core radius. However, such an assumption may not be entirely incorrect. Terman, Taam, \& Hernquist (1995) found that, if the star is sufficiently evolved to produce a steep density gradient between the helium core and the hydrogen envelope, the inspiral of the compact object might halt just above the helium core, preventing the merger of the helium core and compact object. Although their findings were greatly influenced by inadequate resolution $(\sim 3000$ particles $)$ and the subsequent effects of the numerical artificial viscosity inherent in their smooth particle hydrodynamical technique (Rasio \& Livio 1996), nature may still produce similar results. At this time, common envelope evolution is not well enough understood to derive from it any reliable constraints. If we ignore the non-zero helium radius, the rate of DNS and $\mathrm{BH} / \mathrm{NS}$ mergers increases nearly up to $\sim 10^{-4} \mathrm{yr}^{-1}$ (Table 7 ). However, since this particular orbital evolution is currently only justified by an incorrect hydrodynamical simulation, this high merger rate should be considered with suspicion.

Another critical aspect of common envelope evolution is the amount of accretion experienced by a compact object spiraling into the hydrogen envelope of a companion star. In past calculations, it has been assumed that in this state a neutron star will accrete at the Eddington rate. However, studies of the conditions arising near the base of a neutron star suggest that neutron stars in common envelopes will actually accrete at the Bondi-Hoyle rate since the accreting material loses its energy via neutrino emission (Chevalier 1993, 1996; Houck \& Chevalier 1992; Brown 1995; Fryer, Benz, \& Herant 1996). Assuming all neutron stars inspiral to the surface of the expanding star's helium core, Bethe \& Brown (1998) estimated that all neutron stars in common envelope would accrete roughly $1 M_{\odot}$ of material and collapse to form black holes. This collapse can only be avoided only if: angular momentum in the accretion hydrogen is sufficient to lower the accretion rate (Chevalier 1996), neutrino driven explosions eject the accreting hydrogen allowing only small spurts of accretion (Fryer, Benz \& Herant 1996), or the star simply doesn't inspiral to the surface of the helium core where the densities of the hydrogen envelope lead to high temperatures at the surface of the neutron star.

Although angular momentum can halt accretion along the axis, it is unlikely to prevent the rapid accretion onto the neutron star simply because accretion is unimpeded along the poles. Especially at the high densities near the helium core surface (where Bethe \& Brown 1998 predict most of the accretion to occur), it is unlikely that angular momentum will limit the accretion rate sufficiently to prevent neutrino 
cooling (Fryer \& Kalogera 1998). Similarly, although neutrino driven explosions may prevent accretion in the outer layers of a hydrogen giant, near the helium core surface, this explosion mechanism is ineffective (Fryer, Benz, Herant 1996). However, if the neutron star does not inspiral all the way down to the helium core surface, it may not accrete enough to become a black hole and will remain a neutron star. Note that by ignoring hypercritical accretion and assuming that compact objects will not spiral into the helium core produces a DNS merger rate comparable to the rates of past work, most of which made these two, physically unlikely assumptions(Table 7). In addition, these binaries have much shorter orbits on average, and hence shorter merger times. In our standard model, we assumed that all the common envelope neutron stars form black holes and, hence, scenario I (see $\$ 2.1$ ) is excluded from these simulations.

\subsection{Summary and Constraints}

Table 8 summarizes the range of results from all the population synthesis calculations. The basic uncertainties in the rates can be grouped into three categories: kicks, progenitor evolution, and orbital separations. Except for the collapsar model, all the BHAD GRB models depend sensitively (up to three orders of magnitude in the rate) upon the magnitude and distribution of the kick velocities. Uncertainty here dominates the errors in these BHAD GRB rates. On the observational front, additional data on pulsar velocities would be useful. From the theorist we still seek an understanding of why the kicks are so large.

Uncertainties in the progenitor evolution include the mass ratio distribution in binaries, which determines the mass of the secondary and the compact object into which the secondary will evolve, and the mass limits for black hole and collapsar formation. The mass ratio distribution is difficult to determine from first principles, but it better observations could certainly constrain this uncertainty. The mass limit for collapsars on the other hand is something theorists can work on. Together, these uncertainties affect all the GRB rates, and can vary the WD/BH merger and collapsar rates by $\sim 2$ orders of magnitude.

Finally, we have lumped all the uncertainties of binary evolution into the seemingly innocuous category: orbital separation distribution. This category is dominated by uncertainties in the stellar radii and the common envelope evolution. Most of the high DNS rates listed in Lipunov, Postnov, \& Prokhorov (1987) are derived by assuming that neutron stars do not accrete hypercritically and do not merge with their companion's helium core (most previous work used population synthesis codes which do not even determine whether the neutron star spirals into its companion's helium core during a common envelope phase). If we take these assumptions as incorrect and instead rely upon the $\alpha_{\mathrm{CE}}$ prescription for common envelope evolution, the uncertainties in this category result in roughly an order of magnitude decrease in the DNS GRB rates.

Comparison of population synthesis models with observations of compact binary systems can place some constraints upon the allowed parameter space. For instance, in Fig. 19 we plot the simulated distribution of eccentricities and separations of double neutron star systems superimposed on the 4 observed galactic double neutron star systems. Although with so few systems and no knowledge of the observational biases, we do not dare do any statistics on this sample, we can probably rule out very large stellar radii and low kick velocities $\left(v_{\text {kick }}=50\right)$ because such a set of parameters would have a very small probability of producing the observed systems (see Fryer \& Kalogera 1998 for more details).

\footnotetext{
${ }^{2}$ van Kerkwijk \& Kulkarni 1999 have discovered an observational candidate of PSR 2303+46 $\left(A=30 \mathrm{R}_{\odot}, e=0.66\right)$, which would mean that this system consists of a neutron star and a white dwarf and is not a DNS system at all.
} 
The remaining three simulations all reproduce the observed systems, but predict a range of merger rates: $10^{-7} \mathrm{yr}^{-1}<$ MergerRate $_{\mathrm{DNS}}<5 \times 10^{-5} \mathrm{yr}^{-1}$. Similarly, the existence of short-period black hole binaries requires that not all helium stars have the strong winds proposed by Woosley, Langer \& Weaver (1995).

Additional constraints exist and may well provide more stringent limits on the binary formation rate. Until these categories improve and better limits are constructed, we must be satisfied with the rates in Table 8. However, the rates in table 8 provide useful constraints nonetheless. To get the extremely high merger rates of DNS and $\mathrm{BH} / \mathrm{NS}$ binaries, one must assume that compact objects do not merge with their companion's helium core during a common envelope phase. Otherwise, the rate of mergers is at least an order of magnitude lower which, although this decrease may prove troublesome for LIGO and VIRGO, it is certainly sufficient to explain GRBs (see $\S 4$ ). Collapsars and He-mergers also have rates large enough to explain the GRB statistics. It is unlikely that BH/WD mergers explain a sizable fraction of GRBs, but they may be required to explain some peculiar GRBs.

\section{Implications For Observations}

Table 9 summarizes the event rate, distance traveled before producing a burst, and mean redshift for the models we have studied. In addition we give estimates of the burst energy and duration (from other works, e.g., Popham et al. 1999; Meszaros \& Rees 1997a). There is effectively no delay for collapsars and He-mergers which produce bursts occurring, respectively, within $\sim 10 \mathrm{Myr}$ and $\sim 50 \mathrm{Myr}$ of their formation. However most DNS and BH/NS systems experience a considerable delay between their formation and merger and, during that tie, may travel a considerable distance. How far depends upon the mass of the host galaxy. Galaxies similar to the Milky Way have escape velocities of order 400-700 km/s, depending on the location inside the galaxy. The angular separation between GRBs and the centers of their host galaxies is thus an important diagnostic (e.g., Portegies-Zwart \& Yungelson 1998; Bloom, Sigurdsson, \& Pols 1999b; Bulik, Belczyński, \& Zbijewski 1999). There is of course the possibility of a strong selection effect. It might be that GRBs which occur far from their host galaxy are less likely to produce observable afterglows because of the low density of the ISM in galactic halos (Meszaros \& Rees 1997b). This would then mean that we have to multiply the event rates by a corresponding factor much less than one.

In this section, we restrict our analysis to 5 simulations that we feel represent the range of most-likely population synthesis parameters. These include the standard set of parameters, a simulation with winds set to $50 \%$ that of Woosley, Langer \& Weaver (1995), and a simulation with the stellar radii raised to those of Kalogera \& Webbink (1998) all using the FBB kick distribution. Two simulations were run using a Maxwellian kick distribution, one with the standard set of parameters, and one with the stellar radii equal to Kalogera \& Webbink (1998). However the latter of these produced so few binaries that we do not include it in the figures (although the results are in the tables).

\subsection{The angular separation between OTs and host candidates}

There now exists a small, but growing sample of optical GRB afterglows (OTs) associated with host galaxies. In almost every case the angular offset of the OT from the photometric center of the host is of order arcseconds. A linear distance of $10 \mathrm{kpc}$ corresponds to an angular separation of $0.7 \mathrm{~h}_{100} \mathrm{~F}(\mathrm{z}) \operatorname{arcsec}$, where $\mathrm{F} \sim 3$ for redshifts in the range 1-3 (e.g., Peebles 1993) and $\mathrm{h}_{100}$ is the Hubble constant in units of $100 \mathrm{~km} \mathrm{~s}^{-1} \mathrm{Mpc}^{-1}$. Host redshifts are known for GRB970508 $(\mathrm{z}=0.838$; Metzger et al. 1997; Bloom et al. 
1998), GRB971214 ( $\mathrm{z}=3.42$; Kulkarni et al. 1998), GRB980703 ( $\mathrm{z}=0.966$; Djorgovski et al. 1998), and GRB980613 $(\mathrm{z}=1.096$; Djorgovski et al. 1999). These observations clearly argue against any model that invokes massive black holes located at the centers of active or normal galaxies. They also have important implications for models discussed in this paper.

We have modeled the spatial distribution of DNS and BH/NS GRBs by calculating orbits in three representative galactic mass models of Miyamoto-Nagai type (see Hartmann, Epstein, \& Woosley 1990 for details of the dynamic model): a massive galaxy resembling the Milky Way (with $\mathrm{V}_{\text {rot }}\left(\mathrm{R}_{\odot}\right)=220 \mathrm{~km} / \mathrm{s}$ ), a system with a mass four times smaller, and a system with 1/100th the mass of the Milky Way (essentially a negligible mass). The average initial positions of the GRB progenitors in each class were taken to be an axisymmetric ring with galactocentric radius $\mathrm{R}=5 \mathrm{kpc}$ with a Gaussian spread of $1 \mathrm{kpc}$ around that radius. The orbits were following the formation of the primary's compact object. For the DNS and BH/NS binaries, the binaries, this process required two steps, the evolution before and after the collapse of the secondary. Systemic velocities were added as isotropic vectors, while the initial velocity was assumed to be that for circular motion with $\mathrm{V}_{\text {rot }}$. The orbits were followed for a period given from the Monte Carlo population synthesis model. Several thousand orbits were used for each BHAD model. At the moment of final merger the distance to the galactic center was recorded. Fig. 20 shows the resulting distance distribution for 4 different sets of population synthesis parameters. Integrating these curves, it is clear that the models predict a substantial fraction of DNS and BH/NS GRBs at large distances from their hosts (see Fig. 21, Table 10).

In Fig. 21, we plot the distribution of DNS and BH/NS binaries separately, but clearly the two GRB progenitors are very similar reflecting their similar formation history. In addition, because many of the binaries are composed of low-mass black holes (Fig. 17), the velocity boost received to the system as the secondary forms a neutron star is very similar to that received in the case of DNS systems. Our results agree well with those of Bulik, Belczyński, \& Zbijewski (1999) which have a wider spread than that of Bloom et al. (1999b). The population synthesis studies of Bloom et al. (1999b) probably did not include a non-zero helium star radius, allowing them to form DNS binaries with shorter periods, and hence shorter merger times (see $\S 3.4)$.

Fig. 21 also shows the distribution of separations compared to the the observations of OTs corresponding to long hard bursts. It appears that DNS and BH/NS mergers are ruled out as the likely explanation for most of these events. The observations instead support the idea that long duration bursts are either collapsars, helium-mergers, or WD/BH mergers, all of which tend to occur well within the galaxy (see Fig. 21). Indeed, collapsars should all occur at their star formation site. The spread in Fig. 21 reflects only the assumption regarding galactocentric radius for star formation (and is thus also the birth line for the progenitors of DNS and $\mathrm{BH} / \mathrm{NS}$ systems). Ideally this radius should be adjusted to the size of the observed GRB host galaxy, so the agreement with the "promptly dying" models is really better than the figure indicates.

\subsection{GRBs and the Star Formation History of the Universe}

Since only very massive stars are believed to produce black holes, all of our models are in one way or

another dependent upon the star formation history of the universe. Helium-mergers and collapsars trace it most directly since the delay time between star birth and the GRB is, by cosmic standards, instantaneous. The relatively infrequent $\mathrm{WD} / \mathrm{BH}$ mergers may be delayed by a longer time $(\sim 100 \mathrm{Myr})$ but still should 
trace star formation directly. DNS and BH/NS systems, on the other hand have a delay and though they reflect the star formation rate, they do so with a spread of delays, depending especially on the gravitational radiation time scale.

Fig. 22 shows the distribution of DNS+BH/NS merger times for our four different sets of population synthesis parameters. Note that all four have some short-period mergers, but the typical case gives a GRB that lags behind the star formation rate. Fig. 23 shows the GRB redshift history for an Einstein-deSitter universe using two different star formation histories under the functional form given by Watanabe et al. (1998): flattened - $A=3 ., z_{p}=1.5, B=0$. and peaked - $A=1 ., z_{p}=1 ., B=0.5$. The flattened and peaked formation histories represent extremes in the star formation. Recent studies of the cosmic star formation rate indicates that the SFR(z) density function does not turn over past $\mathrm{z} \sim 1.5$ (e.g., Pascarelle, Lanzetta, \& Fernandez-Soto 1998), but instead remains roughly constant to redshifts of $\sim 5$. Beyond a redshift of 5, very little is known about the star formation history. At some redshift, the star formation rate must drop. Differences in the population synthesis models cause considerable scatter in the median redshift of GRBs (Table 11). For a flattened star formation rate, the median redshift varies from 0.85-1.2. Note that the peaked star formation rate leads to much lower median burst redshifts (0.54-0.81). Collapsars and helium-mergers will produce bursts which trace the star formation more directly and hence their median redshifts are higher: 1.8, 0.96 respectively for the flattened, peaked star formation histories (Table 10). The median redshift of collapsar GRBs is likely to be even higher, as its rate increases with decreasing mass loss. The low metallicity stars born at high redshift are likely to have weaker winds and, hence, produce more collapsars.

The current redshift estimates of $\mathrm{z}=1-3$ from the observations (e.g., Hogg and Fruchter 1999) lie within our two star formation rates for all of the GRB models. Unfortunately, the uncertainty in the star formation rate beyond a redshift of 1.5 makes it impossible to rule out any of the models. However, even with the uncertainties in the dominant BHAD model and the population synthesis models, the two star formation rates can be easily distinguished. If an increased sample of GRB redshifts pushes toward a median GRB redshift of 2, then no BHAD GRB model can explain the burst distribution with the peaked star formation history. In this way, GRBs can be used to place constraints on the star formation history.

Another constraint on the star formation history is the fact that no lensed GRBs have been observed (Holz, Miller, \& Quashnock 1999). Using their analysis of constant redshift GRB distributions, we can constrain the GRB histories calculated here. For a flat SFR history combined with our standard model and the FBB kick distribution, only $30 \%$ of the DNS+BH/NS GRBs have redshifts greater than 4, and less than $10 \%$ have redshift greater than 8 . The flat SFR history again must be restricted if the he-mergers and collapsars are the dominant long-duration bursts. The peaked SFR history predicts few lensed GRBs, and hence a lensed event would argue against this star formation history.

Table 11 gives the total number of bursts per day for the three star formation rates. To then determine how many bursts we will observe requires a convolution with the detection efficiencies and the beaming factor, which lower this rate by a factor of 30-1000. Within the uncertainties in the conversion from actual bursts to observed bursts, the star formation history, and the rates, all of the BHAD GRB progenitors can explain the observed bursts. 


\section{BHAD GRB Models}

The merger rates, durations, energies, median redshifts and locations of the BHAD GRB models are summarized in Table 9. Our estimated rates, median redshifts and locations, coupled with the previously calculated energies and durations (Popham, Woosley, \& Fryer 1998; Ruffert \& Janka (1998); Janka, Ruffert, \& Eberl 1998) compare well with the current observational limits. From the data in Table 9, we can construct a general picture of GRB progenitors in which short-duration bursts are dominated by DNS and $\mathrm{BH} / \mathrm{NS}$ mergers, and long-duration bursts are dominated by collapsars and helium mergers. If this picture is correct, we can make several observational predictions which will be tested in the next 10 years. We predict that all long-duration bursts should reside in their host galaxy, whereas some short-duration bursts should occur beyond their host galaxy. In addition, the median redshift of short-duration bursts should be less than that of their long-duration counterparts.

\subsection{Short-Duration Bursts}

DNS and BH/NS binary mergers are capable of producing short duration bursts. Their total rate very likely lies in the range of $0.1-30 \mathrm{Myr}^{-1}$ in the Milky way, which corresponds to an overall rate in the universe of $1-1500$ day $^{-1}$. To determine an observed rate, one must then include the beaming factor and detector efficiencies. Note that this rate is much less than has been predicted in previous calculations (Lipunov, Postnov, \& Prokhorov 1987). Although this lower rate does not prevent DNS and BH/NS binary mergers from playing a major role in explaining GRBs, it has discouraging implications for the predicted rate of gravity wave events detectable with LIGO and VIRGO (for a review, see Finn 1999).

DNS and BH/NS binaries are the only BHAD GRBs which can occur outside of their host galaxy and the only models capable of giving bursts much shorter than one second. Roughly $3-8 \%$ of these bursts occur more than a few arc minutes from a host galaxy of Milky Way mass. This fraction can increase beyond $20 \%$ for lower massed host galaxies. In addition, the median redshift of DNS and BH/NS merger GRBs is less, in some cases significantly less, than that of the median star formation redshift. The current bursts whose locations have been "pinpointed" by BeppoSAX are all long-duration bursts and hence, no constraints can be placed on the locations and redshift of short-duration bursts. However, since none of the observed long-duration bursts have been found outside of their host galaxy, the evidence is beginning to suggest that DNS and BH/NS binaries are limited to short-duration bursts. But much more data is required to draw any firm conclusions from the observations.

DNS and BH/NS binaries also experience a significant delay (up to a Hubble time) between their formation and their merger to form a GRB. This delay causes their median redshift to be lower by $20-50 \%$ than that of stars, and hence, the long-duration gamma-ray bursts.

The major uncertainty in these calculations comes from uncertainties in common envelope evolution: accretion during common envelope evolution, the amount of inspiral that occurs and even stellar radii, which determine if a binary goes into a common envelope. Whereas hypercritical accretion requires a new mechanism for DNS formation (Brown 1995), it creates a new formation mechanism for BH/NS systems (Bethe \& Brown 1998). The inspiral in common envelope helps to produce short-period binaries, but if the inspiraling compact object merges with its companion, it forms a helium-merger GRB, not a DNS or $\mathrm{BH} / \mathrm{NS}$ binary. 


\section{2. $\quad$ Long-Duration Bursts}

Collapsars, helium-mergers, and WD/BH binaries all definitely make long-duration bursts. Because we do not know how many stars will have the necessary angular momentum and mass to produce collapsars, the collapsar rate is particularly uncertain. A relatively conservative estimate comes from employing only Scenario XII (Fig. 13) - the late time merger of two helium stars. Using standard parameters (§3) this mechanism alone gives a daily rate of $400 \mathrm{day}^{-1}$ (Table 3). A very liberal estimate, valid if magnetic fields

do not slow the rotation of the helium core, additionally includes Scenario XI and a comparable number of single star collapsars (Scenario X with low helium core mass loss rates and high red giant mass loss rates). This gives a value about two orders of magnitude larger.

Likewise, since the merger of black holes with helium stars has not been studied in detail, its rate is uncertain (10-4000 day $\left.{ }^{-1}\right)$. The WD/BH binary rate is significantly smaller $\left(0.1-50 \mathrm{day}^{-1}\right)$.

Collapsars and helium-mergers occur in their host galaxy and trace the star formation rate. Hence, any long duration burst occurring outside of its host galaxy would present a problem for the simple picture assuming DNS and BH/NS mergers produce only short-duration bursts. Collapsars and He-mergers also occur nearly simultaneously with the star formation rate and will have a higher median redshift than short-duration bursts (Table 9). These bursts may also provide ideal diagnostics of the high redshift star formation history. A much larger sample of well localized GRBs is essential to test these concepts, and to lend support or to disprove the BHAD scenario for cosmological GRBs.

Just like DNS and BH/NS systems, calculations of helium mergers and WD/BH binaries depend most sensitively upon the common envelope evolution. The uncerainties in collapsars are due primarily to the mass loss and spin rate of their progenitors. The mass loss depends upon stellar winds and the spin rate depends upon the coupling of the core to the stellar envelope.

This research has been supported by NASA (NAG5-2843, MIT SC A292701, and NAG5-8128), the NSF (AST-97-31569), and the US DOE ASCI Program (W-7405-ENG-48). It is a pleasure to thank Alex Heger for his invaluable advice. We are grateful for conversations with Alex Heger, Andrew MacFadyen, Norbert Langer, Bob Popham, and Thomas Janka that helped to elucidate some of the uncertain aspects of gamma-ray bursts, massive stellar evolution and hyperaccreting black holes.

\section{REFERENCES}

Arzoumanian, Z., Cordes, J.M., \& Wasserman, I., 1999, submitted to ApJ, astro-ph/9811323

Bailes, M. 1996, Pulsars: Problems and Progress, ASP Conf. Series, 105, 3, ed. Johnston, Walker, \& Bailes

Belczyński, K., \& Bulik, T., 1998, submitted to A\&A, astro-ph/9901193

Bethe, H., \& Brown, G.E., 1998, ApJ, 506, 780

Blandford, R.D., \& Znajek, R.L., 1977, MNRAS, 179, 433

Bloom, J.S., Djorgovski, S.C., Kulkarni, S.R., \& Frail, D.A., 1998, 507, L25

Bloom, J.S., et al. 1999a, ApJ Letters, submitted

Bloom, J.S., Sigurdsson, S., \& Pols, O. 1999b, MNRAS, in press 
Brandt, N., \& Podsiadlowski, P., 1995, MNRAS, 274, 461

Brown, G.E. 1995, ApJ, 440, 270

Bulik, T., Belczyński, K., \& Zbijewski, W. 1999, submitted to MNRAS, astro-ph/9903407

Burrows, A. \& Woosley, S.E., 1986, ApJ, 308, 680

Cappellaro, E., Turatto, M., Tsvetkov, D. Yu., Bartunov, O. S., Pollas, C., Evans, R., Hamuy, M., 1997, A\&A, 322, 431

Caraveo, P., 1993, ApJ, 415, L111

Chevalier, R.A., 1993, ApJ, 411, L33

Chevalier, R.A., 1996, ApJ, 459, 322

Clark, J.P.A., van den Heuvel, E.P.J., \& Sutantyo, W., 1979, A\&A, 72, 120

Cordes, J.M., Romani, R.W., Lundgren, S.C., 1993, Nature, 362, 133

Cordes, J.M., \& Chernoff, D.F., astro-ph/9707308

Curran, S. J., Lorimer, D. R. 1995, MNRAS, 276, 347

Dalton, W.W., \& Sarazin, C.L. 1995, ApJ, 448, 369

Davies, M.B., Benz, W., Piran, T., Thielemann, F.K., 1994, ApJ, 431, 742

Dewey, R.J. \& Cordes, J., 1987, ApJ, 321, 780

Djorgovski, S. G., Kulkarni, S. R., Bloom, J. S., Goodrich, R., Frail, D. A., Piro, L., \& Palazi, E. 1998, ApJ 508, L17

Djorgovski, S., Kulkarni, S. R., Bloom, J. S., Frail, D. A., Chaffee, F., \& Goodrich, R. 1999, GCN Circ. No 189

Eberl, T., Diploma Thesis, MPA-garching

Eberl, T., Janka, H.-T., Ruffert, M., \& Fryer, C.L., in preparation

Ergma, E., \& van den Heuvel, E.P.J., 1998, A\&A, 331, L29

Finn, L. S. 1999, Lectures given at XXVI SLAC Summer Institute on Particle Physics "Gravity: From the Hubble Length to the Planck Length", August 3-14, 1998 gr-qc/9903107

Fishman, G. J., \& Meegan, C. A. 1995, ARA\&A, 33, 415

Flannery, B.P., \& van den Heuvel, E.P.J., 1975, A\&A, 39, 61

Frail, D.A., Goss, W.M., \& Whiteoak, J.B.Z., 1994, ApJ, 342, 260

Frail, D.A., Kulkarni, S.R., Nicastro, S.R., Feroci, M., \& Taylor, G.B 1997, Nature, 389, 261

Fryer, C.L., Benz, W., \& Herant, M., 1996, ApJ, 460, 801

Fryer, C.L., \& Kalogera, V., 1998, ApJ, 489, 244 
Fryer, C.L., Burrows, A., \& Benz, W., 1998, ApJ, 496, 333

Fryer, C.L., \& Woosley, S.E., 1998, ApJ, 502, L9

Fryer, C. L. 1999, accepted by ApJ

Fryer, C. L., Woosley, S. E., Herant, M., \& Davies, M. B., 1999, accepted by ApJ

Gaensler, B.M, \& Johnston, S., 1995, MNRAS, 273, L73

Garmany, C.D., Conti, P.S., Massey, P., 1980, ApJ, 242, 1063

Hartmann, D. H., Epstein, R. I., \& Woosley, S. E. 1990, ApJ 348, 625

Hartmann, D. H., \& Woosley, S. E. 1995, Advances in Space Research, vol. 15, no. 5, 143

Heger, A. 1998, PhD Thesis, MPA-Garching

Hills D., Bender, P.L., \& Webbink, R.F., 1991, ApJ, 369, 271

Hills, J.G. 1983, ApJ, 267, 322

Hogeveen, S.J., 1990, Ap\&SS, 173,315

Hogg, D. W., \& Fruchter, A. S. 1999, ApJ, in press

Holz, D.E., Miller, M.C., \& Quashnock, J.M., 1999, submitted to ApJ

Houck, J.C., \& Chevalier, R.A., 1991, ApJ, 376, 234

Janka, H.-T., \& Ruffert, M., 1996, A\&A, 306, L33

Janka, H.-T., \& Ruffert, M., \& Eberl, 1998, astro-ph/9810057

Kalogera, V., \& Webbink, R.F., 1998, ApJ, 493, 351

Kalogera, V. 1998, ApJ, 493, 368

Kalogera, V., 1999, accepeted by ApJ

Kaspi, V. M., Manchester, R. N., Johnston, S., Lyne, A. G., D’Amico, N. 1996, AJ, 111, 2028

Katz, J.I., 1997, ApJ, 490, 633

Kobulnicky, H.A., and Skillman, E.D., 1997, ApJ, 489, 636

Kraicheva, Z. T., Popova, E. I., Tutukov, A. V., \& Yungelson, L. R. 1979, Soviet Astron., 56, 520

Kulkarni, S. R., et al. 1998, Nature 393, 35

Lee, W. H., \& Kluzniak, W. 1995, AcA 45, L705

Lipunov, V.M., Postnov, K.A., \& Prokhorov, M.E., 1987, A\&A, 176, L1

Lipunov, V.M., Nazin, S.N., Panchenko, I.E., Postnov, K.A., \& Prokhorov, M.E., 1995, A\&A, 298, 677

Livio, M., Ogilvie, G. I., Pringle, J. E. 1999, ApJ, 512, L100 
Lyne, A.G., Lorimer, D.R., 1994, Nature, 369, 127L

Maeder, A., 1992, A\&A, 264, 105

MacDonald, D.A., Thorne, K.S., Price, R.H., \& Zhang, X.-H., 1986, in "Black Holes, the Membrane Paradigm", Eds. Thorne, K.S., Price, R.H., \& MacDonald, D.A., Yale Univ. Press

MacFadyen, A., \& Woosley, S.E., 1999, accepted by ApJ

Mathews, G.J., Wilson, J.R., 1997, ApJ, 482, 929

Meszaros, P., \& Rees, M. J. 1997, ApJ 476, 232

Meszaros, P., \& Rees, M. J. 1997, ApJ 482, L29

Metzger, M.R., Djorgovski, S.G, Kulkarni, S.R., Steidel, C.C., Adelberger, K.L., Frail, D.A., Costa, E., \& Frontera, F., 1997, Nature, 387, 389

Narayan R., Piran T., Shemi A., 1991, ApJ, 379, L17

Narayan R., Mahadevan, R., \& Quataert, E. 1998, to appear in "The Theory of Black Hole Accretion Discs", eds. M. A. Abramowicz, G. Bjornsson, and J. E. Pringle

Paczynski, B., 1991, AcA, 41, 257

Paczysnki, B. 1998, ApJ 494, L45

Pascarelle, S. M., Lanzetta, K. M., \& Fernandez-Soto, A. 1998, astro-ph/9810060

Peebles, P. J. E., 1993, Principles of Physical Cosmology, Princeton Univ. Press (Princeton)

Phinney E.S., 1991, ApJ, 380, L17

Phinney E.S., \& Kulkarni, S.R., 1994, ARA\&A, 32, 591

Podsiadlowski, P., Joss, P.C., Hsu, J.J.L., 1992, ApJ, 391, 246

Popham, R., Woosley, S. E., \& Fryer, C. L., 1999, accepted by ApJ, astro-ph/9807028

Portegies-Zwart, S.F., \& Spreeuw, H.N., 1996, A\&A, 312, 670

Portegies-Zwart, S.F., Verbunt, F., \& Ergma, E., 1997, A\&A, 321, 207

Portegies-Zwart, S.F., \& Yungelson, L.R., 1998, accepted in A\&A

Rasio, F. A., \& Livio, M. 1996, ApJ, 471, 366

Rasio, F. A., \& Shapiro, S. L., 1994, ApJ, 432, 242

Ruffert, M., Janka, H.-T., Schäfer, G., 1997, A\&A, 311, 532

Ruffert, M., Janka, H.-T., Takahashi, K., Schaefer, G., 1997, A\&A, 319, 122

Ruffert, M., \& Janka, H.-T., 1998, submitted to A\&A

Sandquist, E.L., Taam, R.E., Chen, X., Bodenheimer, P., \& Burkert, A., 1998, accepted by ApJ 
Scalo, J. M. 1986, Fund. of Cosmic Phys., 11, 1

Spruit, H. C., \& Phinney, E. S. 1998, Nature, 393, 193

Terman, J.L., Taam, R.E., \& Hernquist, L., 1995, ApJ, 445, 367

Tutukov, A.V., \& Yungelson, L.R., 1993, MNRAS, 260, 675

van den Heuvel, E.P.J., \& Rappaport, S., 1986, Physics of Be stars, Proc. of the 92nd IAU Coll., 291, Cambridge University Press

van Kerkwijk, M. H., Kulkarni, S. R. 1999, accepted by ApJ

Verbunt, F., 1993, ARA\&A, 31, 93

Wasserman, I., Cordes, J., \& Chernoff, D., 1997, in preparation

Watanabe, K., Hartmann, D.H., Leising, M.D., \& The, L.-S. 1999, in press

Webbink, R.F., 1979, ApJ, 227, 178

Webbink, R.F., 1984, ApJ, 277, 355

Wellstein, S. \& Langer, N. 1999, submitted to A\&A

White, N.E., \& Paradijs, J.V., 1996, ApJ, 473, L25

Wilson, J.R., Mathews, G.J., \& Marronetti, P., 1996, Phys. Rev. D, 54, 1317

Woosley, S.E., 1993, ApJ, 405, 273

Woosley, S.E., Langer, N., \& Weaver, T.A., 1993, ApJ, 411, 823

Woosley, S.E., Langer, N., \& Weaver, T.A., 1995, ApJ, 448, 315

Woosley, S.E., \& Weaver, T.A., 1995, ApJS, 101, 181

Yamaoka, H., Shigeyama, T., Nomoto, K., 1993, ApJ, 267, 433 
Table 1. GRB Formation Scenarios

\begin{tabular}{|c|c|c|c|c|}
\hline Scenario & Common Envelope & Primary Mass ${ }^{\mathrm{b}}$ & Secondary Mass & Comments $^{\mathrm{c}}$ \\
\hline DNS I & NS in Sec. & $M_{\mathrm{SN}}<M_{\mathrm{p}}^{0}<M_{\mathrm{BH}}$ & $M_{\mathrm{SN}}<M_{\mathrm{s}}^{0}<M_{\mathrm{BH}}$ & $\begin{array}{l}\text { Hyp. Acc. } \rightarrow \text { V } \\
\text { Low } \alpha_{\mathrm{CE}} \rightarrow \text { XIII }\end{array}$ \\
\hline II & He Cores & $M_{\mathrm{SN}}<M_{\mathrm{p}}^{0}<M_{\mathrm{BH}}$ & $M_{\mathrm{s}}^{0}=M_{\mathrm{p}}^{0} \pm 5 \%$ & Low $\alpha_{\mathrm{CE}} \rightarrow$ Single \\
\hline III & none & $M_{\mathrm{SN}}<M_{\mathrm{p}}^{0}<M_{\mathrm{BH}}$ & $M_{\mathrm{SN}}<M_{\mathrm{s}}^{0}<M_{\mathrm{BH}}$ & Kick \\
\hline \multirow{2}{*}{$\begin{array}{r}\mathrm{BH} / \mathrm{NS} \mathrm{IV} \\
\mathrm{V}\end{array}$} & $\mathrm{BH}$ in Sec. & $M_{\mathrm{p}}^{0}>M_{\mathrm{BH}}$ & $M_{\mathrm{SN}}<M_{\mathrm{s}}^{0}<M_{\mathrm{BH}}$ & \multirow{3}{*}{$\begin{array}{l}\text { Low } \alpha_{\mathrm{CE}} \rightarrow \text { XIII } \\
\text { no Hyp. Acc. } \rightarrow \text { I } \\
\text { Low } \alpha_{\mathrm{CE}} \rightarrow \text { XIII } \\
\text { Kick }\end{array}$} \\
\hline & NS in Sec. & $M_{\mathrm{SN}}<M_{\mathrm{p}}^{0}<M_{\mathrm{BH}}$ & $M_{\mathrm{SN}}<M_{\mathrm{s}}^{0}<M_{\mathrm{BH}}$ & \\
\hline VI & none & $M_{\mathrm{p}}^{0}>M_{\mathrm{BH}}$ & $M_{\mathrm{SN}}<M_{\mathrm{s}}^{0}<M_{\mathrm{BH}}$ & \\
\hline WD/BH VII & Sec. in Pri. & $M_{\mathrm{p}}^{0}>M_{\mathrm{BH}}$ & $M_{\mathrm{s}}^{0}<M_{\mathrm{SN}}$ & Low $\alpha_{\mathrm{CE}} \rightarrow$ Single \\
\hline VIII & NS in Sec. & $M_{\mathrm{SN}}<M_{\mathrm{p}}^{0}<M_{\mathrm{BH}}$ & $M_{\mathrm{s}}^{0}<M_{\mathrm{SN}}$ & Low $\alpha_{\mathrm{CE}} \rightarrow \mathrm{XIII}$ \\
\hline IX & none & $M_{\mathrm{p}}^{0}>M_{\mathrm{BH}}$ & $M_{\mathrm{s}}^{0}<M_{\mathrm{SN}}$ & Kick \\
\hline Collapsar X & Single Star & $M^{0}>M_{\text {Coll }}$ & $\mathrm{N} / \mathrm{A}$ & Winds \\
\hline XI & Sec. in Pri. & $M^{0}>M_{\text {Coll }}$ & none & Hyd. ejected \\
\hline XII & Double He Cores & $M^{0} \gtrsim M_{\text {Coll }}$ & none & Merger \\
\hline He-Merger XIII & NS,BH in Sec. & $M_{\mathrm{p}}^{0}>M_{\mathrm{SN}}$ & none & $\begin{array}{l}\text { High } \alpha_{\mathrm{CE}} \rightarrow \\
\text { I,IV,V,VII }\end{array}$ \\
\hline
\end{tabular}

${ }^{a}$ Many scenarios require a common envelope phase, one of the most poorly understood aspects of the population synthesis of binary systems. If a common envelope phase exists, hypercritical accretion, the common envelope

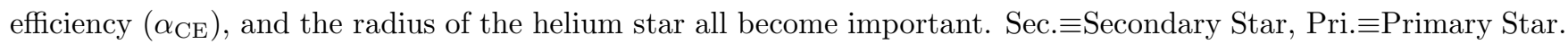

${ }^{\mathrm{b}} M_{\mathrm{SN}} \equiv$ critical mass above which the core of the massive star will collapse to form a neutron star or black hole $\left(M_{\mathrm{SN}} \approx 8-12 M_{\odot}\right) . M_{\mathrm{BH}} \equiv$ critical mass above which the collapse forms a black hole $\left(M_{\mathrm{BH}} \approx 25-35 M_{\odot}\right)$.

${ }^{\mathrm{c}}$ Sensitivity of the scenario on the population synthesis parameters and the inter-relation of the scenarios. Hyp. Acc. $\equiv$ Hypercritical Accretion, $\alpha_{\mathrm{CE}} \equiv$ common envelope efficiency, Single $\equiv$ Single Star, Kick $\equiv$ result extremely sensitive to neutron star kick. A high helium radius has the same effect as a low common envelope efficiency. 
Table 2. Population Synthesis: Initial Conditions

\begin{tabular}{|c|c|c|c|c|c|}
\hline \multirow[b]{2}{*}{ Scenario } & \multicolumn{5}{|c|}{ Formation Rate $\left(\mathrm{Myr}^{-1}\right.$ per galaxy) } \\
\hline & Stand. & $\alpha_{\mathrm{IMF}}=2.35$ & $\alpha_{\mathrm{MR}}=-1.0$ & $\alpha_{\mathrm{MR}}=2.7$ & $P\left(e_{0}\right) \propto 1$ \\
\hline \multicolumn{6}{|c|}{ 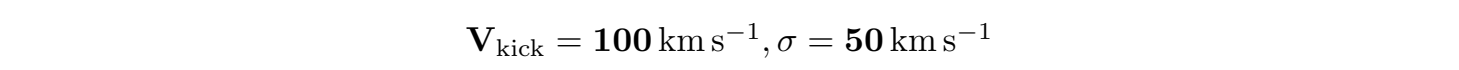 } \\
\hline DNS II ${ }^{\mathrm{a}}$ & 3.2 & 4.2 & 8.9 & 0.14 & 3.4 \\
\hline III & 0.0012 & 0.00069 & 0.0019 & 0.00026 & 0.0 \\
\hline Total & 3.2 & 4.2 & 8.9 & 0.14 & 3.4 \\
\hline BH/NS IV & 1.7 & 2.6 & 1.6 & 0.59 & 0.93 \\
\hline $\mathrm{V}$ & 10. & 14. & 13. & 3.2 & 7.1 \\
\hline VI & 0.00029 & 0.00034 & 0. & 0.00026 & 0. \\
\hline Total & 12. & 16. & 14. & 3.8 & 8.1 \\
\hline WD/BH VII & 0.18 & 0.22 & 0.10 & 0.24 & 0.22 \\
\hline VIII & 0.0032 & 0.0021 & 0.00063 & 0.0065 & 0.0023 \\
\hline IX & 0.18 & 0.27 & 0.085 & 0.41 & 0.44 \\
\hline Total & 0.36 & 0.49 & 0.19 & 0.66 & 0.44 \\
\hline Collapsar X & 5.0 & 7.5 & 17 & 0.18 & 5.6 \\
\hline XI & 620. & 1100. & 880. & 130. & 590. \\
\hline XII & 0. & 0. & 5.1 & 0. & 0. \\
\hline Total & 630. & 1100. & 900. & 130. & 600. \\
\hline He-Merg. XIII & 39. & 44. & 34. & 29. & 34. \\
\hline \multicolumn{6}{|c|}{ 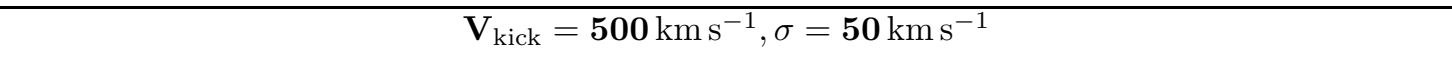 } \\
\hline DNS II ${ }^{\mathrm{a}}$ & 0.31 & 0.30 & 0.86 & 0.010 & 0.32 \\
\hline III & 0. & 0. & 0. & 0. & 0. \\
\hline Total & 0.31 & 0.30 & 0.86 & 0.010 & 0.32 \\
\hline BH/NS IV & 0. & 0. & 0. & 0. & 0. \\
\hline $\mathrm{V}$ & 0.11 & 0.18 & 0.12 & 0.11 & 0.096 \\
\hline VI & 0.0020 & 0.0016 & 0.0032 & 0. & 0.0010 \\
\hline Total & 0.11 & 0.18 & 0.12 & 0.11 & 0.097 \\
\hline WD/BH VII & 0.0014 & 0.0020 & 0.00040 & 0.0068 & 0.00040 \\
\hline VIII & 0.00040 & 0.00040 & 0.00040 & 0.0024 & 0.00060 \\
\hline IX & 0.0058 & 0.0070 & 0.00020 & 0.019 & 0.0050 \\
\hline Total & 0.0076 & 0.0094 & 0.0010 & 0.028 & 0.0060 \\
\hline Collapsar X & 4.7 & 7.3 & 16 & 0.18 & 5.3 \\
\hline XI & 600. & 1100. & 840. & 130. & 570. \\
\hline XII & 0. & 0. & 5.4 & 0.12 & 0. \\
\hline Total & 600. & 1100. & 860. & 130. & 580. \\
\hline He-Merg. XIII & 2.4 & 2.8 & 3.3 & 1.1 & 2.8 \\
\hline
\end{tabular}

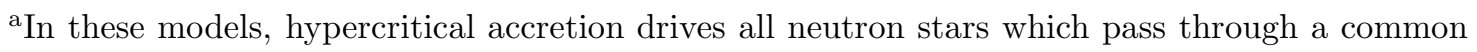
envelope phase into black holes. Thus, no DNS binaries are formed via Scenario I. 
Table 3. Population Synthesis: Supernovae

\begin{tabular}{|c|c|c|c|c|c|c|c|c|}
\hline \multirow{3}{*}{$\begin{array}{c}\text { Scenario } \\
V_{\text {kick }}{ }^{c}\end{array}$} & & & \multicolumn{4}{|c|}{ Formation Rate $\left(\mathrm{Myr}^{-1}\right.$ per galaxy) } & \multirow{2}{*}{\multicolumn{2}{|c|}{$M_{\mathrm{BH}}=M_{\text {primary }}{ }^{\mathrm{b}}$}} \\
\hline & \multicolumn{2}{|c|}{ Standard } & \multicolumn{2}{|c|}{$V_{\mathrm{Kick}}^{\mathrm{BH}}=0.1 V_{\mathrm{Kick}}^{\mathrm{NS}}$} & \multicolumn{2}{|c|}{$M_{\mathrm{BH}, \mathrm{Coll}}^{\mathrm{crit}}=75,90 M_{\odot}^{\mathrm{a}}$} & & \\
\hline & FBB & Maxw. & 100 & 500 & 100 & 500 & 100 & 500 \\
\hline DNS II & 1.2 & 0.97 & 3.6 & 0.59 & 2.8 & 0.14 & 3.1 & 0.61 \\
\hline III & 0. & 0. & 0.0015 & 0 . & 0.00083 & 0. & 0.0015 & 0. \\
\hline Total & 1.2 & 0.7 & 3.6 & 0.59 & 2.8 & 0.14 & 3.2 & 0.61 \\
\hline $\mathrm{BH} / \mathrm{NS}$ IV & 0.55 & 0.057 & 1.0 & 3.6 & 0.068 & 0. & 4.9 & 0.0075 \\
\hline $\mathrm{V}$ & 2.6 & 0.20 & 6.6 & 1.9 & 8.0 & 0.001 & 14. & 0.091 \\
\hline VI & 0.00029 & 0. & 0. & 0. & 0. & 0. & 0.0058 & 0. \\
\hline Total & 3.1 & 0.26 & 7.6 & 5.5 & 8.1 & 0.001 & 19. & 0.98 \\
\hline WD/BH VII & 0.071 & 0.017 & 0.18 & 0.25 & 0. & 0. & 0.18 & 0. \\
\hline VIII & 0.00087 & 0.0012 & 0.0035 & 0. & 0. & 0. & 0. & 0. \\
\hline IX & 0.081 & 0.016 & 0.18 & 0.25 & 0. & 0. & 0.0099 & 0.0017 \\
\hline Total & 0.15 & 0.034 & 0.36 & 0.5 & 0. & 0. & 0.19 & 0.0017 \\
\hline Collapsar X & 4.5 & 4.6 & 4.5 & 4.8 & 0.44 & 0.012 & 4.6 & 5.0 \\
\hline XI & 620. & 610. & 600 & 590. & 140. & 30. & 600. & 620. \\
\hline XII & 5.8 & 0.9 & 1.5 & 0. & 0.46 & 0.06 & 0. & 0. \\
\hline $\begin{array}{r}\text { Total } \\
\text { He-Merg. XIII }\end{array}$ & $\begin{array}{l}630 . \\
14 .\end{array}$ & $\begin{array}{l}620 . \\
3.0\end{array}$ & $\begin{array}{l}610 . \\
35 .\end{array}$ & $\begin{array}{l}590 . \\
15 .\end{array}$ & $\begin{array}{l}140 . \\
23 .\end{array}$ & $\begin{array}{l}30 . \\
0 .\end{array}$ & $\begin{array}{l}600 . \\
46 .\end{array}$ & $\begin{array}{l}630 . \\
0.35\end{array}$ \\
\hline
\end{tabular}

${ }^{a}$ This is the critical initial stellar mass above which massive stars form black holes. The higher number corresponds to the stellar mass required to collapse to a black hole without the launch of a supernova shock. This higher number is required to form collapsars. In our standard models, we assume that stars above $25 M_{\odot}$ collapse to form black holes and stars with mass above $40 M_{\odot}$ do not launch supernova shocks.

${ }^{\mathrm{b}} \mathrm{A}$ star may explode as a supernova but have sufficient fallback to drive its later collapse into a black hole. Thus, the resultant mass may not equal the star's mass just prior to its collapse. Our standard model assumes that the star is $1 / 3$ the collapsing star's mass.

${ }^{\mathrm{c}}$ We give several kick distributions. FBB stands for the Fryer, Burrows, Benz (1998) kick distribution: 40\% with $V_{\text {mean }}=100 \mathrm{~km} \mathrm{~s}^{-1}$ and $60 \%$ with $V_{\text {mean }}=600 \mathrm{~km} \mathrm{~s}^{-1}$. Maxw. stands 1for a Maxwellian distribution with a mean of $450 \mathrm{~km} \mathrm{~s}^{-1}$ The other velocities refer to the mean velocity using our smoothed $\left(\sigma=50 \mathrm{~km} \mathrm{~s}^{-1}\right)$ delta-function kick distributions. 
Table 4. Population Synthesis: Stellar Evolution

\begin{tabular}{|c|c|c|c|c|c|c|c|}
\hline \multirow{3}{*}{$\begin{array}{c}\text { Scenario } \\
V_{\text {kick }}\end{array}$} & \multicolumn{7}{|c|}{ Formation Rate $\left(\mathrm{Myr}^{-1}\right.$ per galaxy) } \\
\hline & \multicolumn{2}{|c|}{ Standard } & \multicolumn{2}{|c|}{$R_{\text {star }}=4 \times R_{\text {star }}^{\text {standard }}$} & \multirow{2}{*}{$\begin{array}{c}\alpha_{\text {wind }}=0 \\
\text { FBB }\end{array}$} & \multirow{2}{*}{$\begin{array}{c}\alpha_{\text {wind }}=0.5 \\
\text { FBB }\end{array}$} & \multirow{2}{*}{$\begin{array}{c}\alpha_{\text {wind }}=1 \\
\text { FBB }\end{array}$} \\
\hline & 100 & 500 & 100 & 500 & & & \\
\hline DNS II & 3.2 & 0.61 & 0.60 & 0.013 & 1.2 & 0.91 & 2.2 \\
\hline III & 0.0012 & 0. & 0.00029 & 0. & 0 . & 0. & 0 . \\
\hline Total & 3.2 & 0.61 & 0.60 & 0.013 & 1.2 & 0.91 & 0.22 \\
\hline BH/NS IV & 1.7 & 0.00029 & 0.90 & 0. & 0.59 & 0.072 & 0.019 \\
\hline $\mathrm{V}$ & 10. & 0.009 & 3.4 & 0.0014 & 2.6 & 1.1 & 0.090 \\
\hline VI & 0.00029 & 0. & 0. & 0. & 0. & 0. & 0. \\
\hline Total & 12. & 0.0093 & 4.3 & 0.0014 & 3.2 & 1.2 & 0.11 \\
\hline WD/BH VII & 0.18 & 0. & 0.85 & 0.00029 & 0.026 & 0.28 & 0.00020 \\
\hline VIII & 0.0032 & 0. & 0.0069 & 0 & 0.0035 & 0.00028 & 0. \\
\hline IX & 0.18 & 0.00058 & 0.36 & 0. & 0.15 & 0.066 & 0. \\
\hline Total & 0.36 & 0.00058 & 1.2 & 0.00029 & 0.18 & 0.35 & 0.00020 \\
\hline Collapsar X & 4.7 & 5.0 & 0.91 & 0.93 & 6.8 & 0.19 & 0.38 \\
\hline XI & 600 & 620. & 600. & 610. & 670. & 450. & 0. \\
\hline XII & 0. & 0. & 4.0 & 3.4 & 5.8 & 6.0 & 5.0 \\
\hline Total & 600. & 630. & 600 & 610. & 680 & 460. & 5.4 \\
\hline He-Merg. XIII & 39. & 0.12 & 140. & 0.046 & 14. & 16. & 6.2 \\
\hline
\end{tabular}


Table 5. Population Synthesis: Mass Transfer

\begin{tabular}{rlllll}
\hline \hline \multicolumn{7}{c}{ Formation Rate $\left(\mathbf{M y r}^{-1}\right.$} & per galaxy), $V_{\text {kick }}=$ FBB & \\
Scenario & $\beta_{\mathrm{MT}}=1.0$ & $\begin{array}{l}\alpha_{\mathrm{MT}}=0.5 \\
\beta_{\mathrm{MT}}=0.8\end{array}$ & $\begin{array}{l}\alpha_{\mathrm{MT}}=2.0 \\
\beta_{\mathrm{MT}}=0.8\end{array}$ & $\begin{array}{l}\alpha_{\mathrm{MT}}=0.5 \\
\beta_{\mathrm{MT}}=0.5\end{array}$ & $\begin{array}{l}\alpha_{\mathrm{MT}}=2.0 \\
\beta_{\mathrm{MT}}=0.5\end{array}$ \\
\hline DNS II & 1.2 & 1.2 & 1.2 & 1.2 & 1.2 \\
III & 0. & 0.00029 & 0.00058 & 0.00029 & 0. \\
Total & $\mathbf{1 . 2}$ & $\mathbf{1 . 2}$ & $\mathbf{1 . 2}$ & $\mathbf{1 . 2}$ & $\mathbf{1 . 2}$ \\
BH/NS IV & 1.8 & 0.95 & 0.16 & 0.094 & 0.054 \\
V & 8.1 & 3.9 & 1.2 & 0.76 & 0.62 \\
VI & 0. & 0. & 0. & 0. & 0. \\
Total & $\mathbf{9 . 9}$ & $\mathbf{4 . 9}$ & $\mathbf{1 . 4}$ & $\mathbf{0 . 8 5}$ & $\mathbf{0 . 6 7}$ \\
WIII & 0.069 & 0.071 & 0.073 & 0.073 & 0.074 \\
IX & 0.0012 & 0.00087 & 0.0012 & 0.00088 & 0.0012 \\
Total & $\mathbf{0 . 1 6}$ & 0.079 & 0.079 & 0.081 & 0.083 \\
Collapsar X & 5.1 & $\mathbf{0 . 1 5}$ & $\mathbf{0 . 1 5}$ & $\mathbf{0 . 1 6}$ & $\mathbf{0 . 1 6}$ \\
XI & 710. & 4.5 & 4.4 & 3.5 & 3.0 \\
XII & 6.0 & 650. & 540. & 500. & 170. \\
Total & $\mathbf{7 2 0 .}$ & 5.9 & 5.5 & 5.5 & 5.2 \\
He-Merg. XIII & $\mathbf{3 6 .}$ & $\mathbf{6 6 0 .}$ & $\mathbf{5 5 0 .}$ & $\mathbf{5 1 0 .}$ & $\mathbf{1 8 0 .}$ \\
\hline
\end{tabular}


Table 6. Population Synthesis: Common Envelope Efficiency

\begin{tabular}{|c|c|c|c|c|c|c|}
\hline \multirow{3}{*}{$\begin{array}{c}\text { Scenario } \\
V_{\text {kick }}\end{array}$} & \multicolumn{6}{|c|}{ Formation Rate $\left(\mathrm{Myr}^{-1}\right.$ per galaxy) } \\
\hline & \multicolumn{2}{|c|}{ Standard: $\alpha_{\mathrm{CE}}=0.5$} & \multicolumn{2}{|c|}{$\alpha_{\mathrm{CE}}=0.25$} & \multicolumn{2}{|c|}{$\alpha_{\mathrm{CE}}=1.0$} \\
\hline & 100 & 500 & 100 & 500 & 100 & 500 \\
\hline DNS II & 3.2 & 0.61 & 3.3 & 0.78 & 3.8 & 0.51 \\
\hline III & 0.0012 & 0 . & 0.0012 & 0 . & 0.00058 & 0 . \\
\hline Total & 3.2 & 0.61 & 3.3 & 0.78 & 3.8 & 0.51 \\
\hline BH/NS IV & 1.7 & 0.00029 & 2.0 & 0.00058 & 1.4 & 0.00058 \\
\hline $\mathrm{V}$ & 10. & 0.0090 & 4.9 & 0.0058 & 10.0 & 0.007 \\
\hline VI & 0.00029 & 0 . & 0 . & 0. & 0.00058 & 0 . \\
\hline Total & 12. & 0.0093 & 6.9 & 0.0064 & 11. & 0.0076 \\
\hline WD/BH VII & 0.18 & 0. & 0.022 & 0.00029 & 0.54 & 0.00029 \\
\hline VIII & 0.0032 & 0. & 0.0029 & 0. & 0.018 & 0. \\
\hline IX & 0.18 & 0.00058 & 0.078 & 0.0017 & 0.29 & 0 . \\
\hline Total & 0.36 & 0.00058 & 0.10 & 0.0020 & 0.86 & 0.00029 \\
\hline Collapsar X & 4.7 & 5.0 & 4.5 & 4.6 & 4.5 & 4.7 \\
\hline XI & 600. & 620. & 580. & 600. & 630. & 650. \\
\hline XII & 0. & 0. & 1.3 & 1.2 & 1.6 & 1.4 \\
\hline $\begin{array}{r}\text { Total } \\
\text { He-Merg. XIII }\end{array}$ & $\begin{array}{l}600 . \\
39 .\end{array}$ & $\begin{array}{l}630 . \\
0.12\end{array}$ & $\begin{array}{l}590 . \\
33 .\end{array}$ & $\begin{array}{l}610 . \\
0.091\end{array}$ & $\begin{array}{l}640 . \\
49 .\end{array}$ & $\begin{array}{l}660 . \\
0.038\end{array}$ \\
\hline
\end{tabular}


Table 7. Population Synthesis: Hypercritical Accretion

\begin{tabular}{|c|c|c|c|c|c|c|c|c|}
\hline \multirow{3}{*}{$\begin{array}{c}\text { Scenario } \\
V_{\text {kick }}\end{array}$} & \multicolumn{8}{|c|}{ Formation Rate $\left(\mathrm{Myr}^{-1}\right.$ per galaxy) } \\
\hline & \multicolumn{2}{|c|}{ Standard } & \multicolumn{2}{|c|}{ No HA } & \multicolumn{2}{|c|}{ No He-Mrg. } & \multicolumn{2}{|c|}{ No He-Mrg./HA } \\
\hline & 100 & 500 & 100 & 500 & 100 & 500 & 100 & 500 \\
\hline DNS I & 0 . & 0 . & 6.6 & 0.0081 & 0 . & 0. & 20. & 0.018 \\
\hline II & 3.2 & 0.61 & 3.2 & 0.61 & 63. & 15 . & 63. & 15. \\
\hline III & 0.0012 & 0 . & 0.0015 & 0. & 0.00058 & 0. & 0.0015 & 0 . \\
\hline Total & 3.2 & 0.61 & 9.8 & 0.62 & 63. & 15. & 83. & 15. \\
\hline BH/NS IV & 1.7 & 0.00029 & 1.0 & 0.00029 & 11. & 0.22 & 9.9 & 0.021 \\
\hline $\mathrm{V}$ & 10. & 0.0090 & 0. & 0. & 26. & 0.019 & 0. & 0. \\
\hline VI & 0.00029 & 0. & 0 . & 0. & 0.00029 & 0. & 0.00029 & 0 . \\
\hline Total & 12. & 0.0093 & 1.0 & 0.00029 & 37. & 0.040 & 10. & 0.021 \\
\hline WD/BH VII & 0.18 & 0 . & 0.18 & 0. & 0.19 & 0.0012 & 0.19 & 0.0012 \\
\hline VIII & 0.0032 & 0. & 0.0032 & 0. & 0.0035 & 0. & 0.0035 & 0. \\
\hline IX & 0.18 & 0.00058 & 0.18 & 0.00058 & 0.18 & 0.00087 & 0.18 & 0.00087 \\
\hline Total & 0.36 & 0.00058 & 0.36 & 0.00058 & 0.37 & 0.0020 & 0.37 & 0.0020 \\
\hline Collapsar X & 4.7 & 5.0 & 4.7 & 5.0 & 4.4 & 4.6 & 4.4 & 4.6 \\
\hline XI & 600. & 620. & 600. & 620. & 610. & 630. & 610. & 630. \\
\hline XII & 0. & 0. & 0. & 0. & 0. & 0. & 0. & 0. \\
\hline $\begin{array}{r}\text { Total } \\
\text { He-Merg. XIII }\end{array}$ & $\begin{array}{l}600 . \\
39 .\end{array}$ & $\begin{array}{l}630 . \\
0.12\end{array}$ & $\begin{array}{l}600 . \\
39 .\end{array}$ & $\begin{array}{l}630 . \\
0.12\end{array}$ & $\begin{array}{l}610 . \\
0 .\end{array}$ & $\begin{array}{l}630 . \\
0 .\end{array}$ & $\begin{array}{l}610 . \\
0 .\end{array}$ & $\begin{array}{l}630 . \\
0 .\end{array}$ \\
\hline
\end{tabular}

Table 8. Formation Rate Summary

\begin{tabular}{rcc}
\hline \hline model & $\begin{array}{c}\text { Range } \\
\left(\mathrm{Myr}^{-1} \text { per gal. }\right)^{\mathrm{a}}\end{array}$ & $\begin{array}{c}\text { Standard Parameters } \\
\left(\mathrm{Myr}^{-1} \text { per gal. }\right)^{\mathrm{a}}\end{array}$ \\
\hline DNS & $0.01-80$ & 1.2 \\
BH/NS & $0.001-50$ & 3.1 \\
WD/BH & $0.0001-1$ & 0.15 \\
Collapsar & $\sim 10-1000$ & 680 \\
He-Merg. & $\sim 0.1-50$ & 14 \\
& & \\
\hline
\end{tabular}

${ }^{a}$ The Formation Rate is for a galactic supernova rate of $0.02 \mathrm{yr}^{-1}$. 
Table 9. BHAD GRB Models

\begin{tabular}{rllllll}
\hline \hline model & $\begin{array}{c}\text { Form. Rate } \\
(\mathrm{GRB} / \mathrm{day})^{\mathrm{b}}\end{array}$ & $\begin{array}{c}\text { Log } \mathrm{E}_{\nu \bar{\nu}}^{\mathrm{a}} \\
(\mathrm{Erg})\end{array}$ & $\begin{array}{c}\text { Log } \mathrm{E}_{\text {Mag. }} \\
(\mathrm{Erg})\end{array}$ & $\begin{array}{c}\text { Duration a } \\
(\mathrm{s})\end{array}$ & $\begin{array}{c}\text { Fraction } \\
>1 \mathrm{Mpc}^{\mathrm{c}}\end{array}$ & $\begin{array}{l}\mathrm{z}_{G R B}^{\text {median }} \\
\left(\mathrm{z}_{S N}^{\text {median }}\right)\end{array}$ \\
\hline DNS & $1-300$ & $49.7-50.5$ & $\lesssim 53$ & $\sim 0.1 \mathrm{~s}$ & $3-8,17-39 \%$ & $0.5-0.8$ \\
BH/NS & $1-1200$ & $50-51$ & $\lesssim 53$ & $\sim 0.1 \mathrm{~s}$ & $3-8,17-39 \%$ & $0.5-0.8$ \\
WD/BH & $0.1-50$ & $\lesssim 50$ & $\lesssim 53$ & $15-150 \mathrm{~s}$ & $0,0 \%$ & $\lesssim 1$ \\
Collapsar & $100-5 . \times 10^{4}$ & $\lesssim 52$ & $\lesssim 53$ & $10 \mathrm{~s}$ & $0,0 \%$ & $\gtrsim 1$ \\
He-Merg. & $10-4 \times 10^{3}$ & $\lesssim 50$ & $\lesssim 53$ & $15-500 \mathrm{~s}$ & $0,0 \%$ & 1 \\
& & & & & & \\
\hline
\end{tabular}

${ }^{a}$ The energies and durations are taken from Popham, Woosley, \& Fryer(1998), Ruffert \& Janka (1998), or Janka, Ruffert, \& Eberl (1998). These models assumed high viscous forces. The durations of the DNS and BH/NS GRBs could be much higher if the viscosity is lower (Meszaros \& Rees 1997a).

${ }^{b}$ The formation rates are based on a flat star formation history with a $\mathrm{z}=5$ cut-off and a limited set of parameter values to produce the "most-likely" range.

${ }^{\mathrm{c}}$ We list values for galaxies with respective masses equal to and 1/4th that of the Milky Way.

Table 10. DNS+BH/NS Relation to Host Galaxy ${ }^{\mathrm{a}}$

\begin{tabular}{|c|c|c|c|c|c|c|}
\hline \multirow[t]{2}{*}{ Model } & \multicolumn{2}{|c|}{$M_{\text {host }}=M_{\mathrm{MW}}$} & \multicolumn{2}{|c|}{$M_{\text {host }}=0.25 M_{\mathrm{MW}}$} & \multicolumn{2}{|c|}{$M_{\text {host }}=0.01 M_{\mathrm{MW}}$} \\
\hline & $>100 \mathrm{kpc}^{\mathrm{b}}$ & $>1 \mathrm{Mpc}$ & $>100 \mathrm{kpc}$ & $>1 \mathrm{Mpc}$ & $>100 \mathrm{kpc}$ & $>1 \mathrm{Mpc}$ \\
\hline Stan., FBB Kick & $13 \%$ & $3.6 \%$ & $30 \%$ & $8.8 \%$ & $56 \%$ & $17 \%$ \\
\hline High Wind, FBB Kick & $9.7 \%$ & $3.4 \%$ & $24 \%$ & $8.6 \%$ & $71 \%$ & $21 \%$ \\
\hline Large R, FBB Kick & $11 \%$ & $4.1 \%$ & $37 \%$ & $13 \%$ & $77 \%$ & $39 \%$ \\
\hline Stan., Maxw. Kick & $25 \%$ & $7.2 \%$ & $39 \%$ & $14 \%$ & $44 \%$ & $17 \%$ \\
\hline Large R, Maxw. Kick & $21 \%$ & $8.2 \%$ & $52 \%$ & $23 \%$ & $62 \%$ & $30 \%$ \\
\hline
\end{tabular}

${ }^{a} \mathrm{WD} / \mathrm{BH}$, helium-merger, and collapsar GRBs all occur within their host galaxy.

${ }^{\mathrm{b}}$ The columns list the percentage of DNS and BH/NS binaries which merge beyond 100,1000 kpc respectively. 
Table 11. DNS+BH/NS Redshift Distribution and Rates ${ }^{\text {a }}$

\begin{tabular}{rcccc}
\hline \hline \multicolumn{1}{c}{ Model } & \multicolumn{2}{c}{ Flat $^{\mathrm{b}}$} & \multicolumn{2}{c}{ Peaked $^{\mathrm{c}}$} \\
& median z & $\begin{array}{c}\text { Rate } \\
\text { day }\end{array}$ & $\begin{array}{c}\text { Rate } \\
\text { day }^{-1}\end{array}$ \\
\hline $\begin{array}{r}\text { Stan., FBB Kick } \\
\text { High Wind, FBB Kick }\end{array}$ & 1.2 & 540 & 0.71 & 53 \\
Large R, FBB Kick & 0.97 & 380 & 0.57 & 36 \\
Stan., Maxw. Kick & 1.5 & 128 & 0.54 & 12 \\
Large R, Maxw. Kick & 1.2 & 156 & 0.81 & 16 \\
& & 24 & 0.71 & 2.3 \\
\hline
\end{tabular}

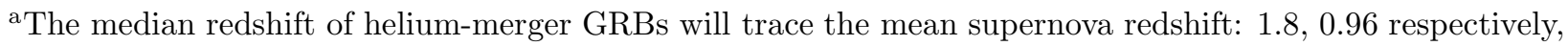
for the flattened and peaked supernova histories. The median redshift of collapsar GRBs will be even larger due to the weaker winds at high redshifts (low metallicities). For the flat star formation history, the supernova, he-merger, collapsar rates are, respectively, $1.3 \times 10^{6}, 900, \sim 4 \times 10^{4}$ per day. The corresponding rates for a peaked star formation history are $1.3 \times 10^{5}, \sim 90, \sim 4000$ per day.

${ }^{\mathrm{b}}$ Flat: $\mathrm{A}=3.0, \mathrm{~B}=0.0, \mathrm{z}_{\mathrm{p}}=1.5$

${ }^{c}$ Peaked: $\mathrm{A}=1.0, \mathrm{~B}=0.5, \mathrm{z}_{\mathrm{p}}=1.0$ 


\section{BHAD GRB Progenitors: Scenarios}

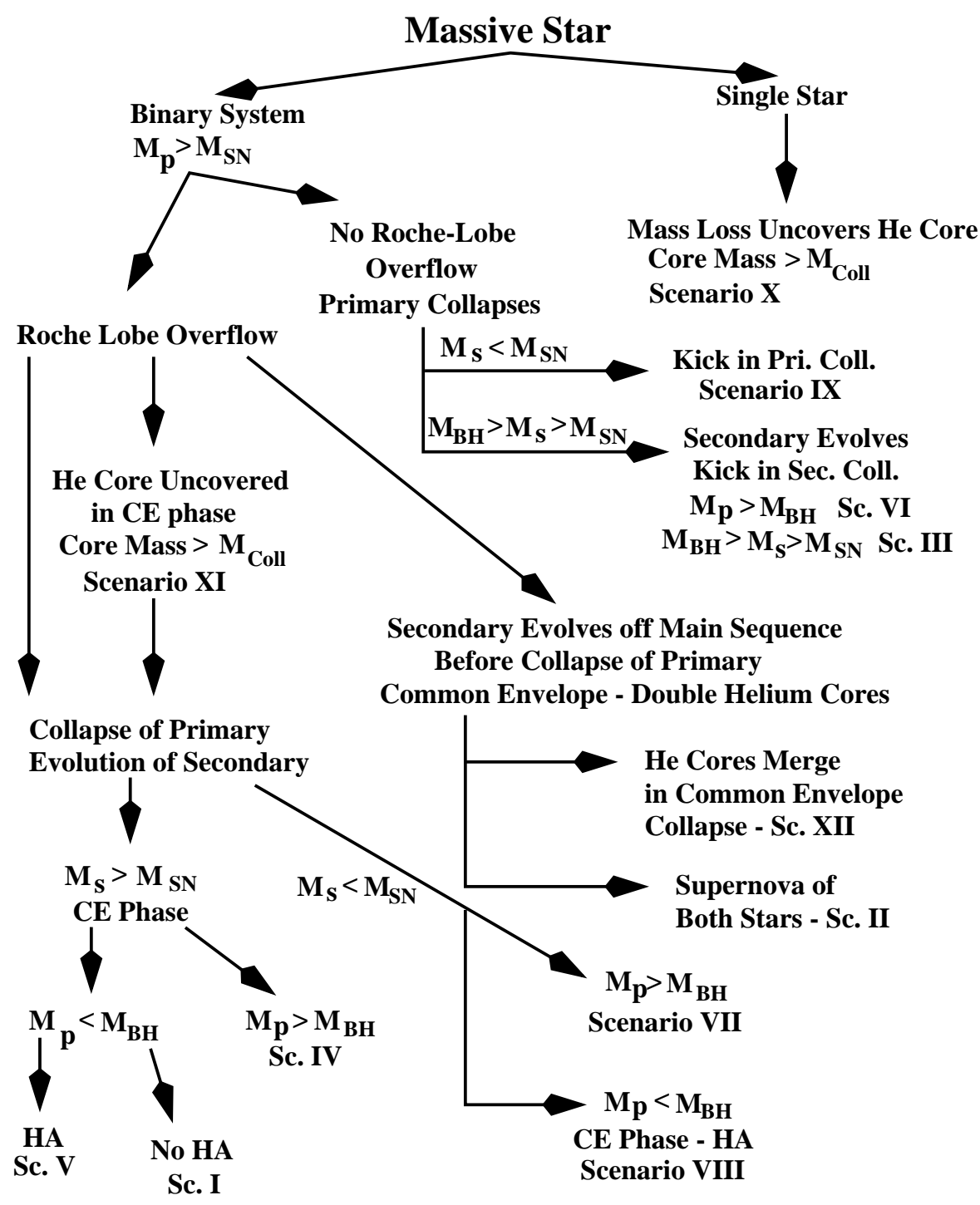

Fig. 1.- Summary of the BHAD GRB formation scenarios studied in this paper. Many of the scenarios are quite similar. For instance, scenarios III, VI, and IX are differentiated simply by the initial mass of the companion stars. Scenarios I and IV differ only by the mass of their primary star. Scenarios I and V are identical except that, for the case of scenario I, we assume that photons limit the accretion onto the neutron star and, for scenario $\mathrm{V}$, hypercritical accretion occurs. The helium merger models are not listed here but occur when a compact object (either black hole or neutron star) merges with its helium companion during a common envelope phase. We use the following abbreviations: $M_{\mathrm{p}}, M_{\mathrm{s}}$ are the primary and secondary masses respectively, $M_{\mathrm{SN}} \approx 10 M_{\odot}$ are the critical masses above which a massive stars collapse to form supernovae, $M_{\mathrm{BH}} \approx 25 M_{\odot}$ is the critical mass where massive stars form black holes, $M_{\mathrm{Coll}} \approx 40 M_{\odot}$ is the mass of helium cores above which no explosion occurs and the entire star collapses to form a black hole, CE indicates a common envelope phase, and HA stands for hypercritical accretion. 

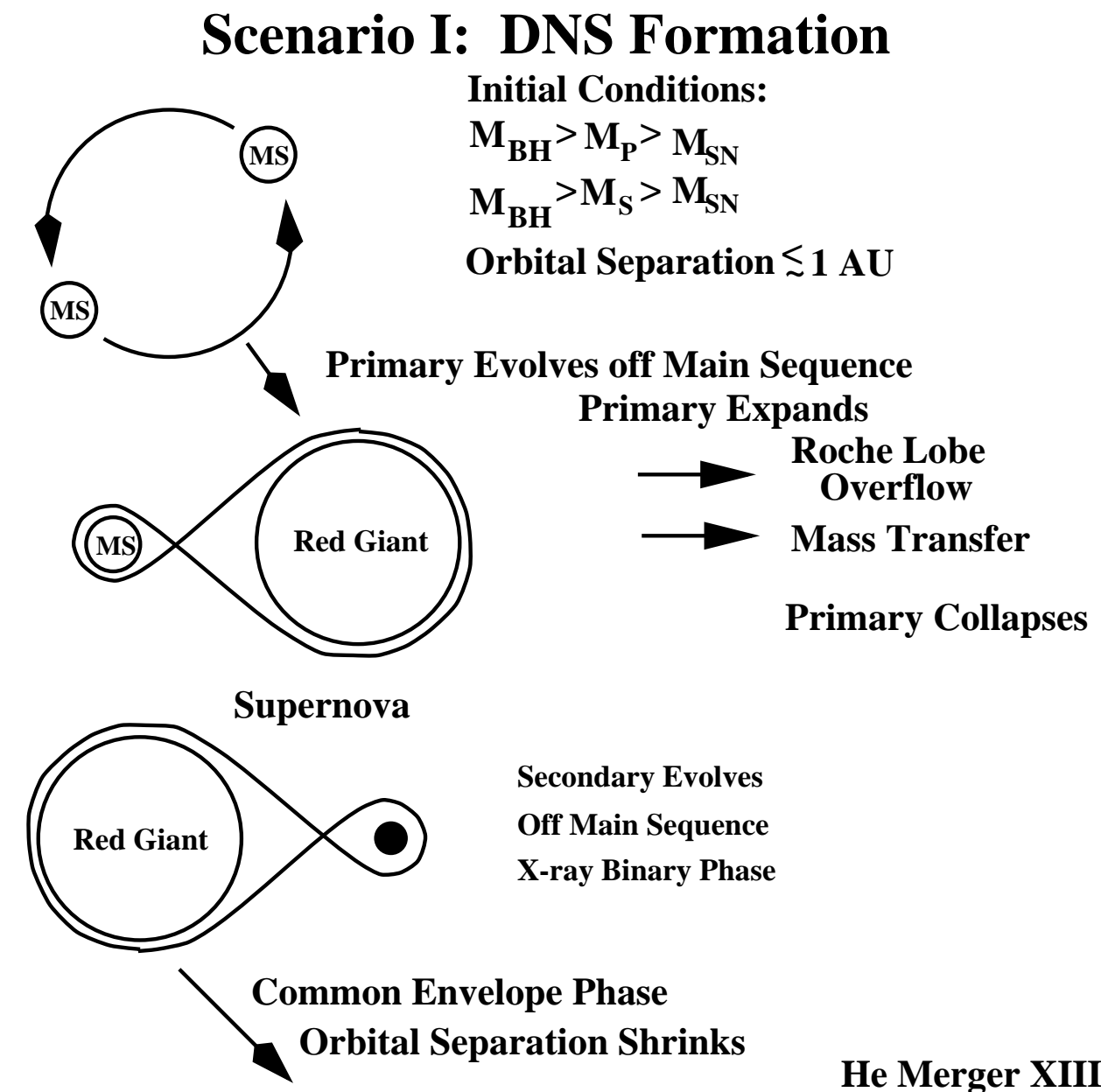

He Merger XIII

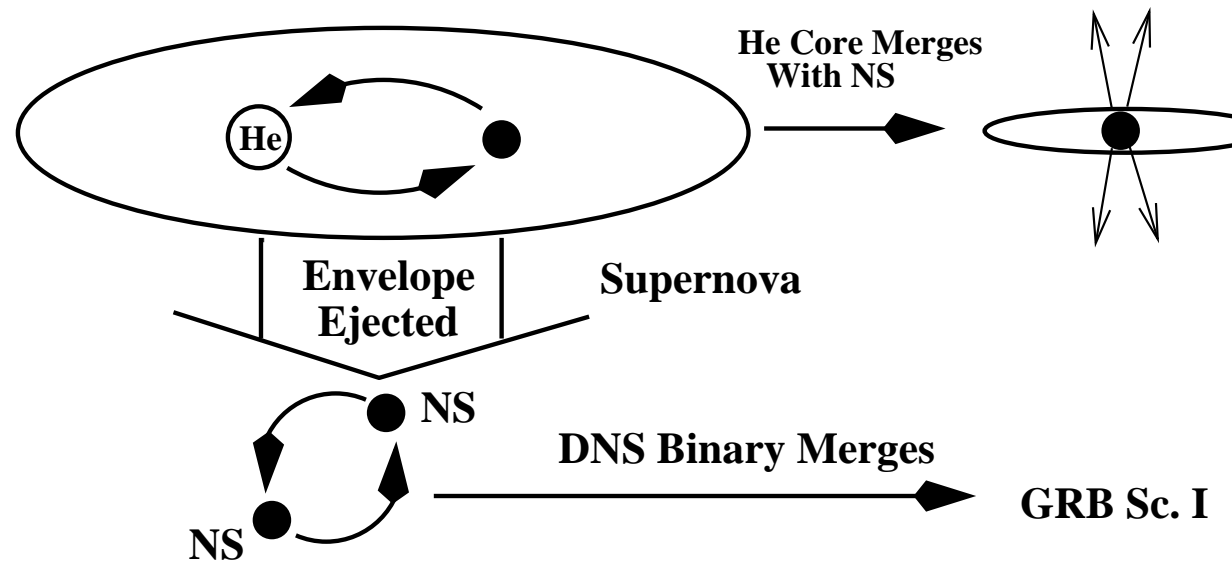

Fig. 2.- Scenario I: the "standard" double neutron star formation scenario. All symbols are as described in Fig. 1, MS denotes a main-sequence star, NS and BH are neutron stars and black holes respectively. Note that if the neutron star merges with its helium companion in the common envelope phase, a He-merger GRB is produced. This scenario assumes the accretion onto the neutron star during this phase is limited to the photon Eddington rate. 

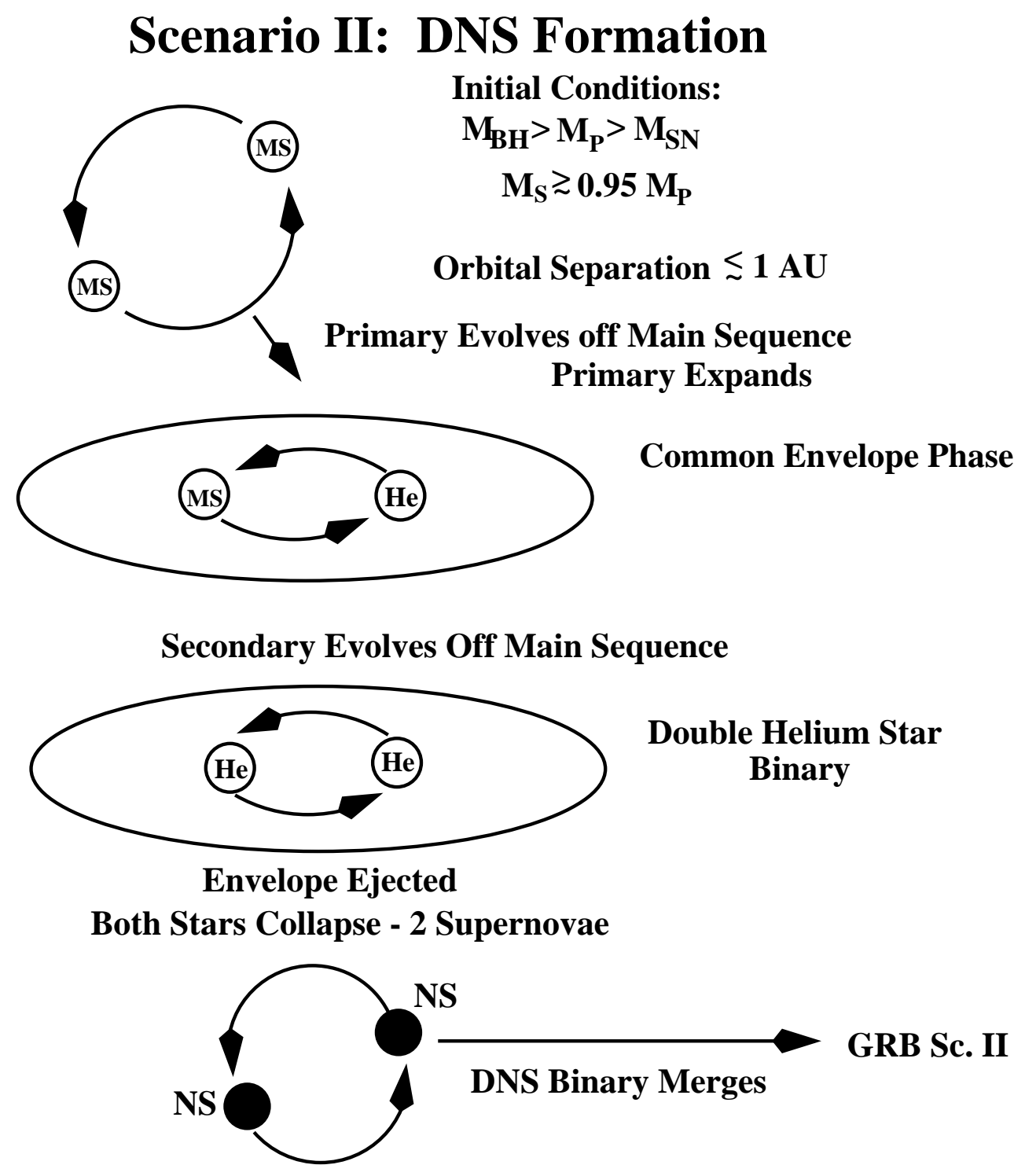

Fig. 3.- Scenario II: The "Brown" mechanism for forming DNS binaries (Brown 1995). All symbols are as described in Figs. 1 and 2. In this phase, the two stars have nearly the same mass and the secondary evolves off the main sequence before the primary collapses, forming a double helium star binary. The collapse of the helium cores form a DNS binary. 

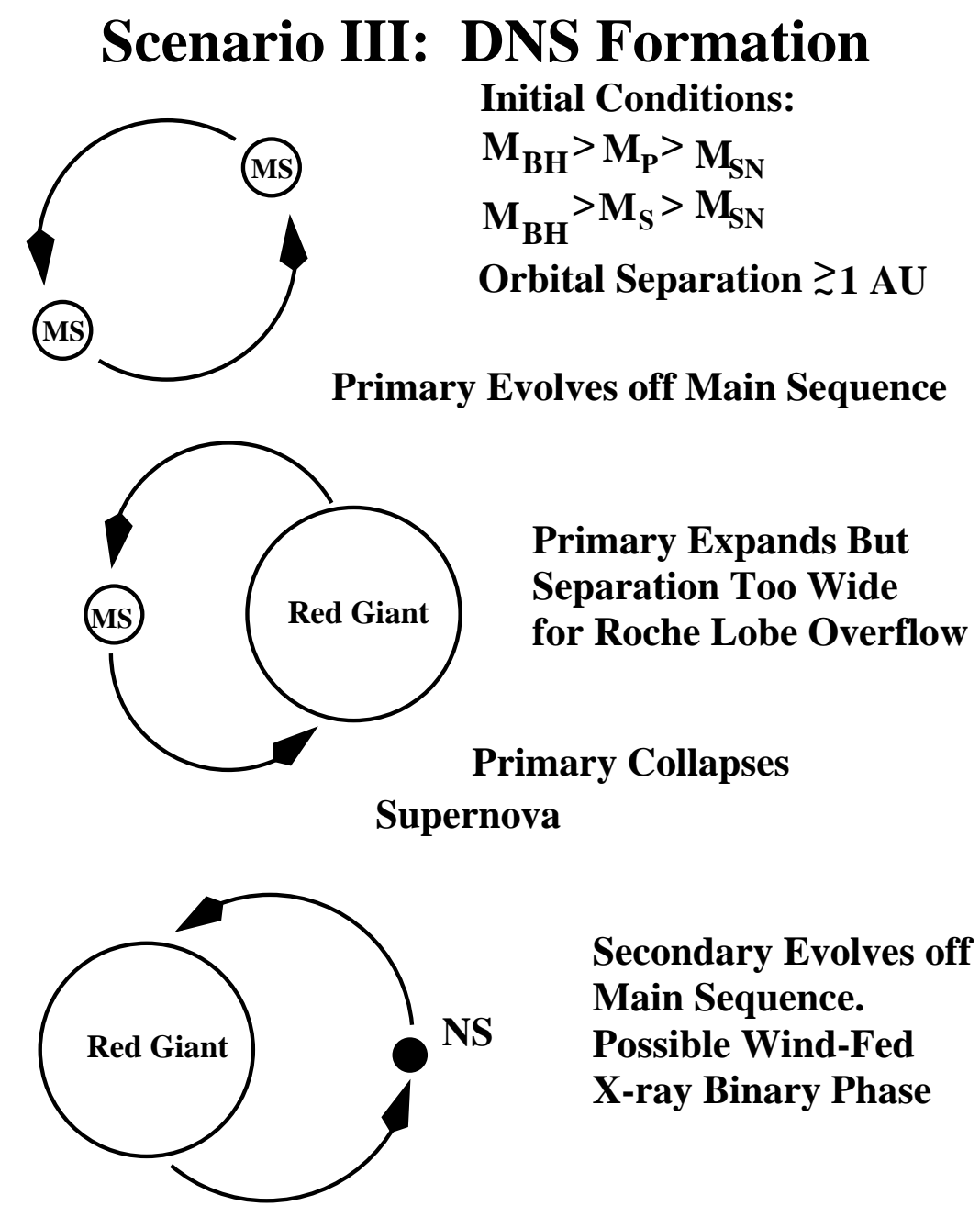

Secondary Evolves off

Main Sequence.

Possible Wind-Fed

X-ray Binary Phase

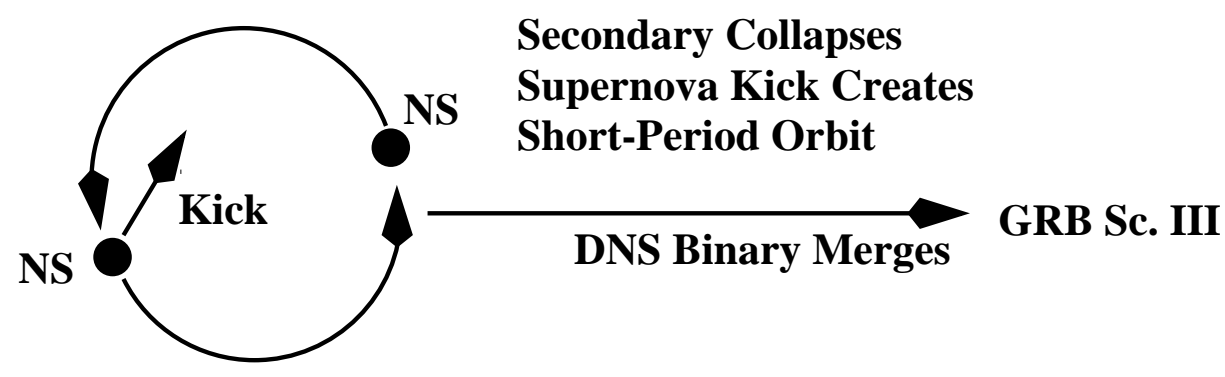

Fig. 4. - Scenario III: The kick scenario for DNS binaries. In this scenario, the binary stars avoid any common envelope phase. All symbols are as described in Figs. 1 and 2. Only a small range of kicks can tighten the orbit and allow the DNS binary to merge within a Hubble time. 


\section{Scenario IV: BH/NS Formation}
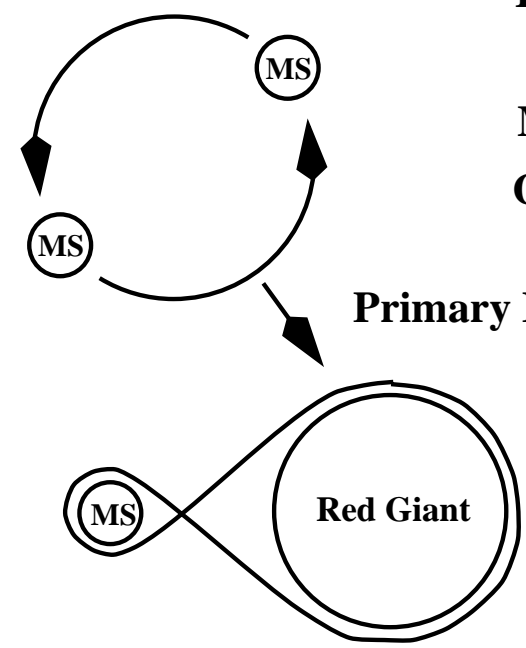

Primary Evolves off Main Sequence

Primary Expands

$\mathbf{M}_{\mathbf{B H}}>\mathbf{M}_{\mathrm{S}}>\mathbf{M}_{\mathrm{SN}}$

Orbital Separation $\lesssim 1 \mathrm{AU}$

Collapse to BH (Possible SN)

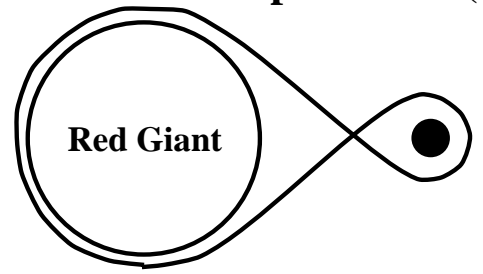

Secondary Evolves

Off Main Sequence

X-ray Binary Phase
Common Envelope Phase Orbital Separation Shrinks

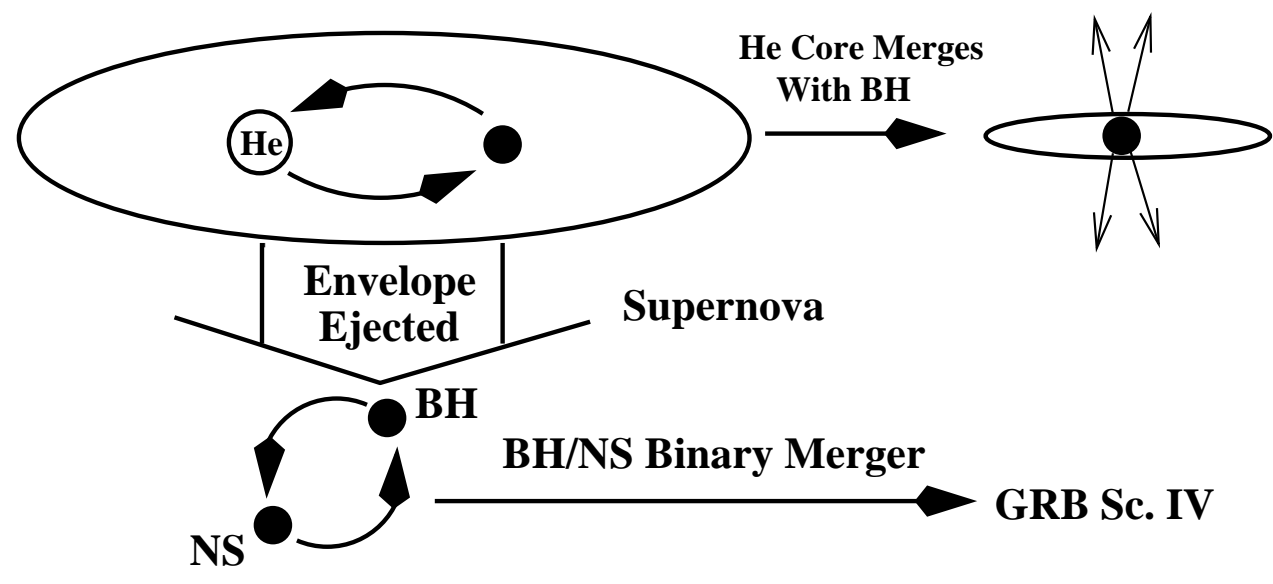

Fig. 5.- Scenario IV: The "standard" BH/NS binary formation phase. This scenario is identical to Scenario I (Fig. 2) except that the primary mass $\left(M_{\mathrm{p}}\right)$ is greater than the critical mass for black hole formation. 


\section{Scenario V: BH/NS Formation}
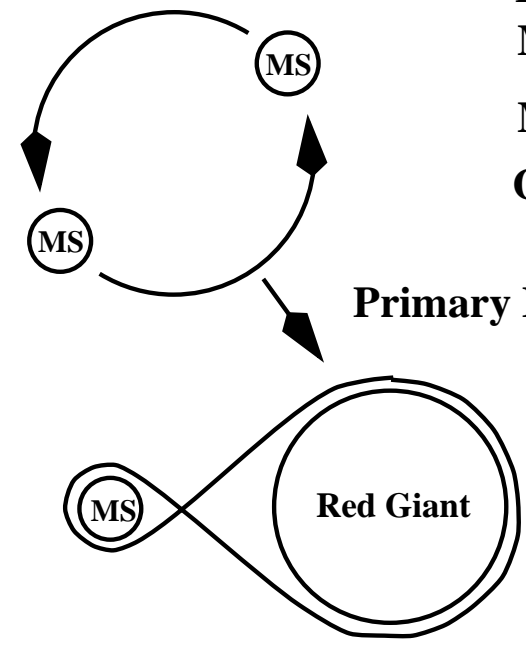

Supernova

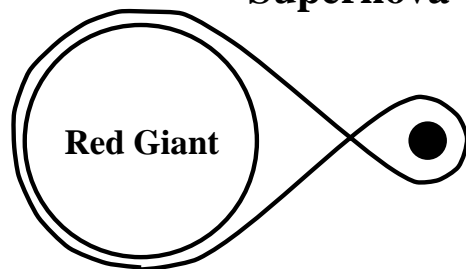

Common Envelope Phase

Orbital Separation Shrinks

Hypercritical Accretion
Initial Conditions:

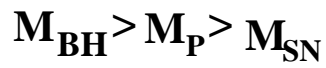

$\mathbf{M}_{\mathrm{BH}}>\mathbf{M}_{\mathrm{S}}>\mathbf{M}_{\mathrm{SN}}$

Orbital Separation $\lesssim 1 \mathrm{AU}$

Secondary Evolves

Off Main Sequence

X-ray Binary Phase
Roche Lobe

Overflow

Mass Transfer

Primary Collapses
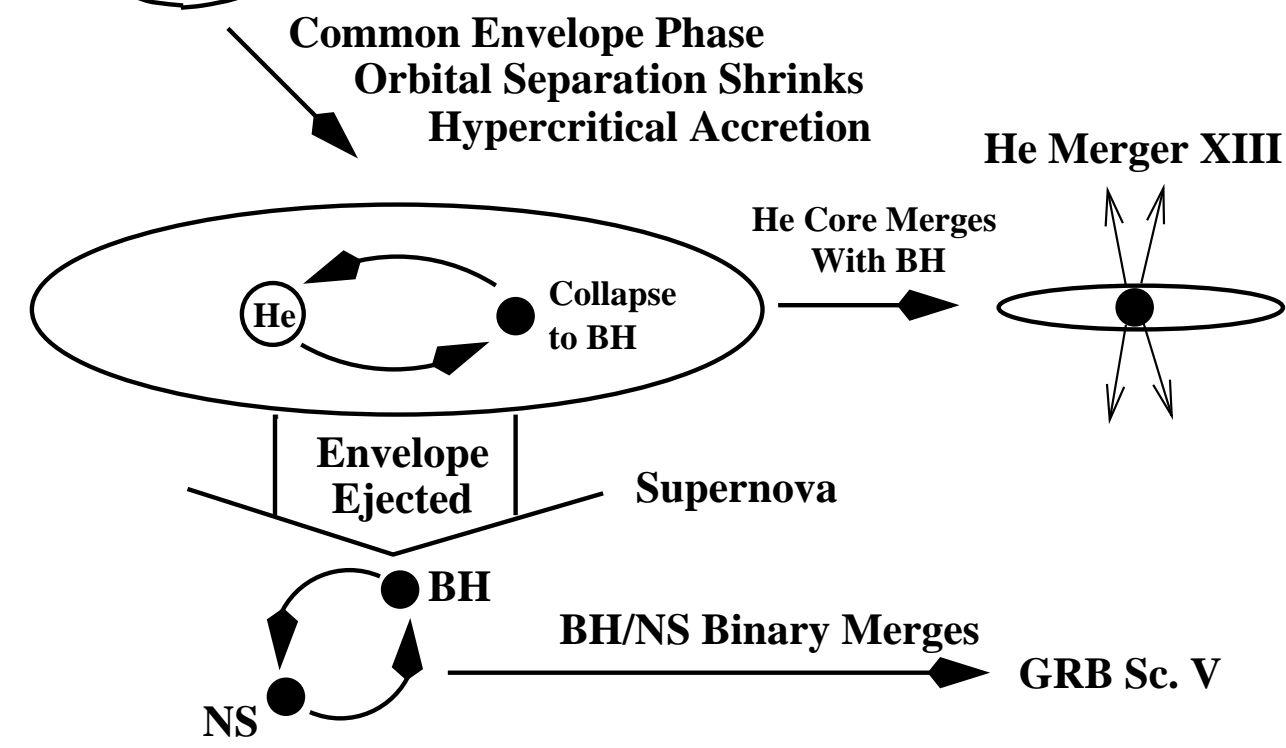

Fig. 6.- Scenario V: low-mass black hole + neutron star binary formation scenario. This scenario is identical to Scenario I (Fig. 2) except that hypercritical occurs during the common envelope phase, causing the neutron star to collapse into a black hole. 

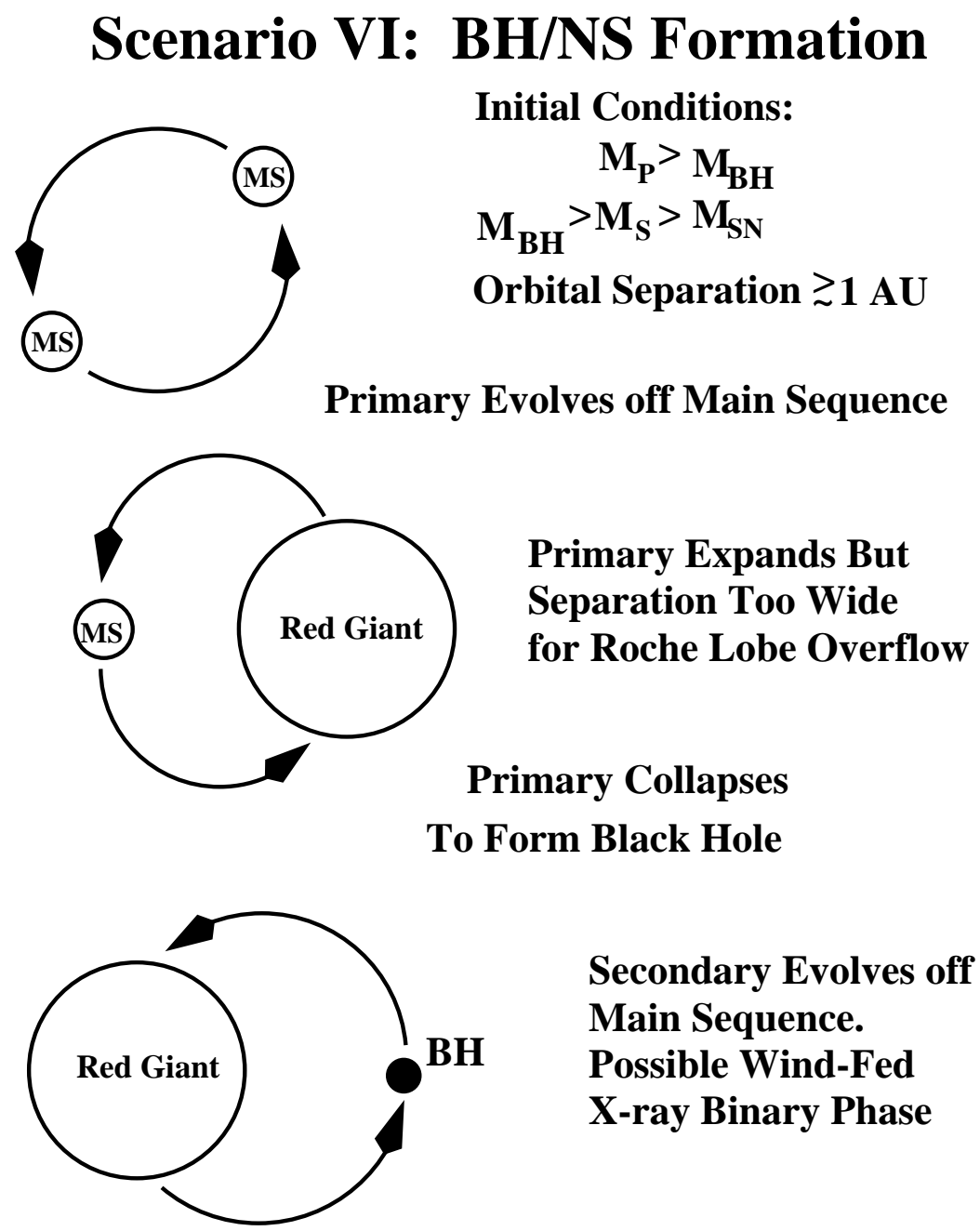

Secondary Evolves off Main Sequence. Possible Wind-Fed X-ray Binary Phase

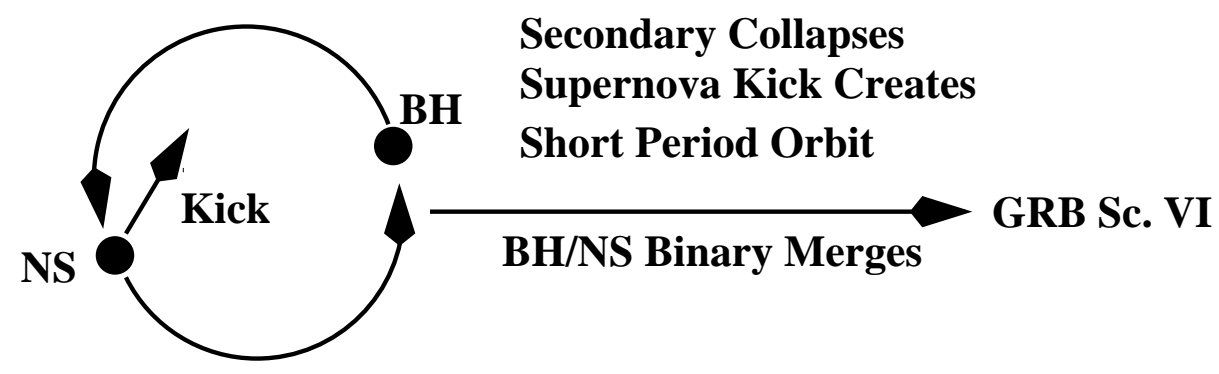

Fig. 7.- Scenario VI: The kick scenario for BH/NS binaries. Identical to scenario III (Fig. 4) except the primary mass is less than the critical black hole formation mass. 


\section{Scenario VII: WD/BH Formation}

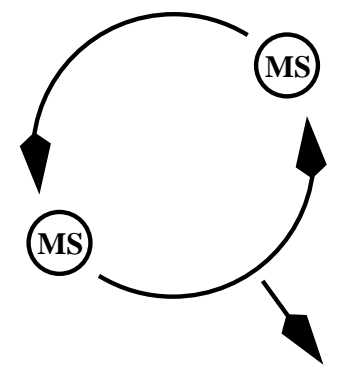

\section{Initial Conditions:}

$$
\begin{aligned}
& M_{\mathbf{P}}>M_{B H} \\
& M_{S}<M_{S N}
\end{aligned}
$$

Orbital Separation $\lesssim 1 \mathrm{AU}$

Primary Evolves off Main Sequence

Primary Expands

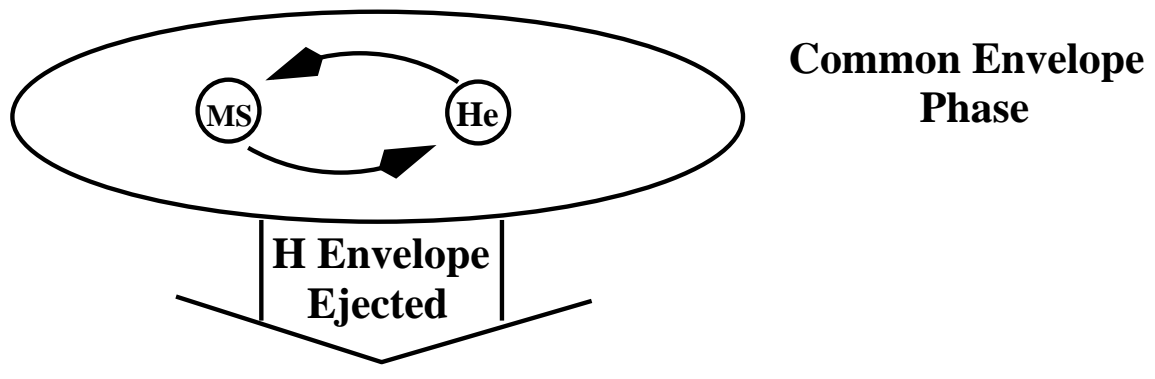

Collapse of Primary (Possible SN)

Secondary Evolves off Main Sequence

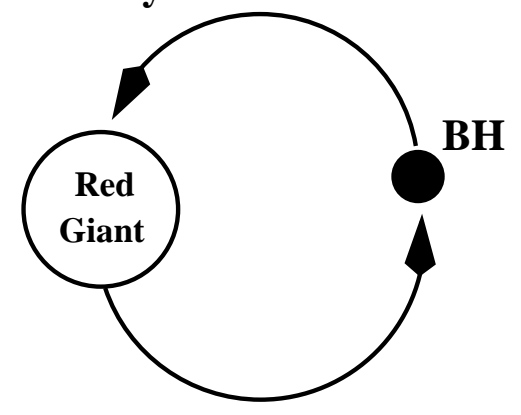

Possible Roche-Lobe Overflow

Low-Mass X-ray Binary

Seconary Sheds Mass and

Becomes White Dwarf

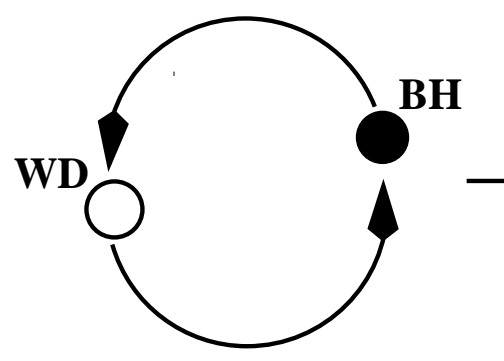

GRB Sc. VII

\section{WD/BH Binary Merges}

Fig. 8.- Scenario VII: "standard" WD/BH formation scenario. This scenario probably produces the intermediate mass X-ray binaries such as LMC X-3. All symbols are described in Figs. 1 and 2. 


\section{Scenario VIII: WD/BH Formation}
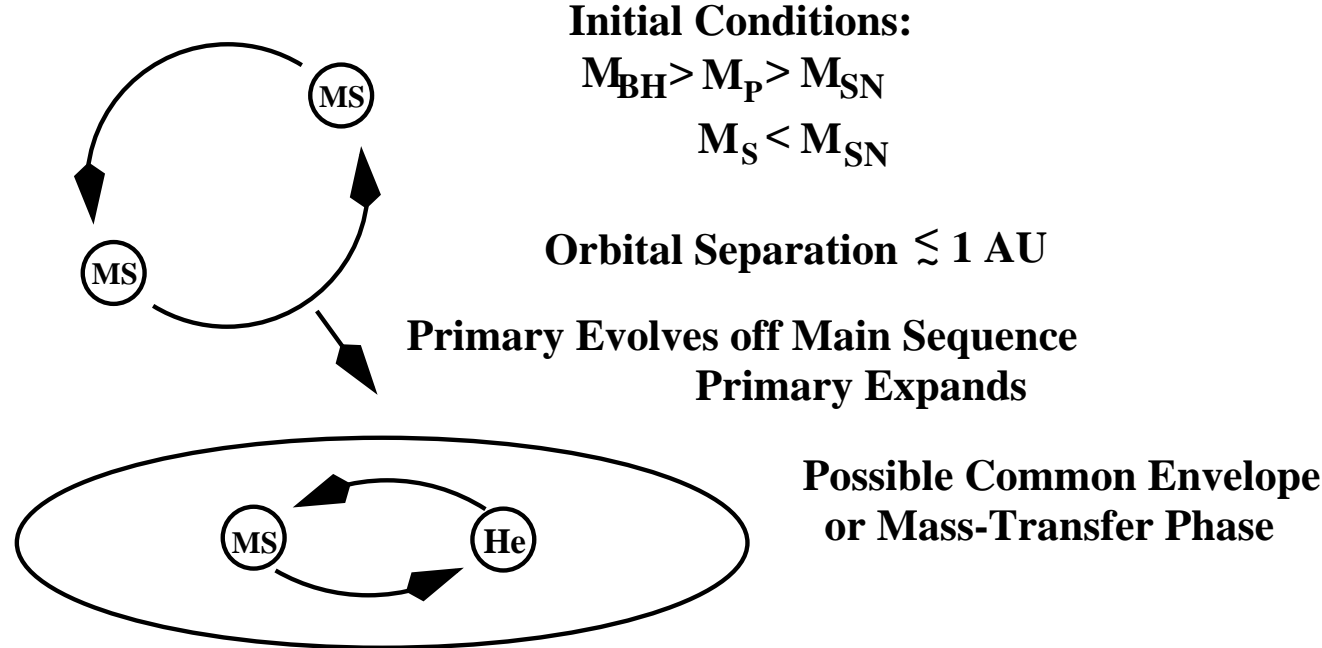

Supernova

Secondary Evolves off Main Sequence

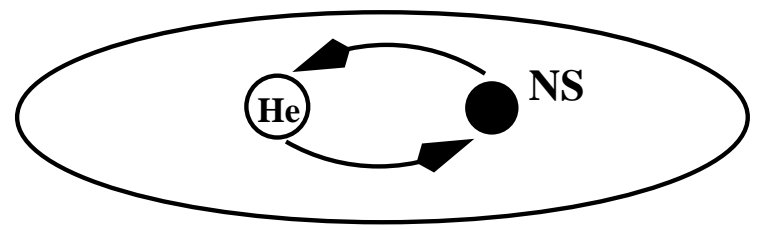

Common Envelope

Phase

Neutron Star Accretes and Collapses to Black Hole

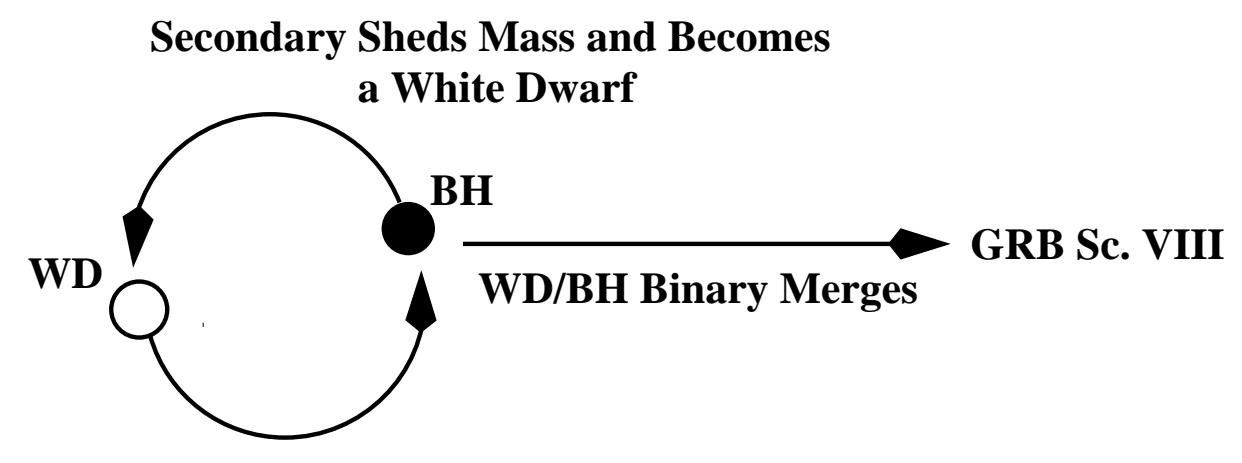

Fig. 9.- Scenario VIII: The hypercritical accretion scenario for WD/BH binaries. This scenario is identical to scenario V (Fig. 6) except that the secondary forms a white dwarf instead of a neutron star. Not that in the common envelope phase, the neutron star can merge and form a helium merger. 


\section{Scenario IX: WD/BH Formation}

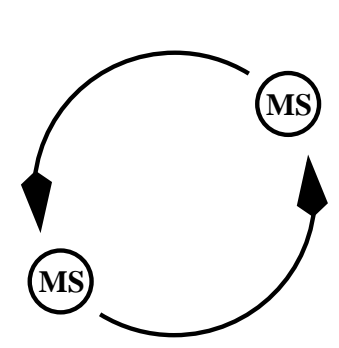

Initial Conditions:

$$
\begin{aligned}
& M_{\mathbf{P}}>M_{B H} \\
& M_{S}>M_{S N}
\end{aligned}
$$

Orbital Separation $\gtrsim 1 \mathrm{AU}$

Primary Evolves off Main Sequence

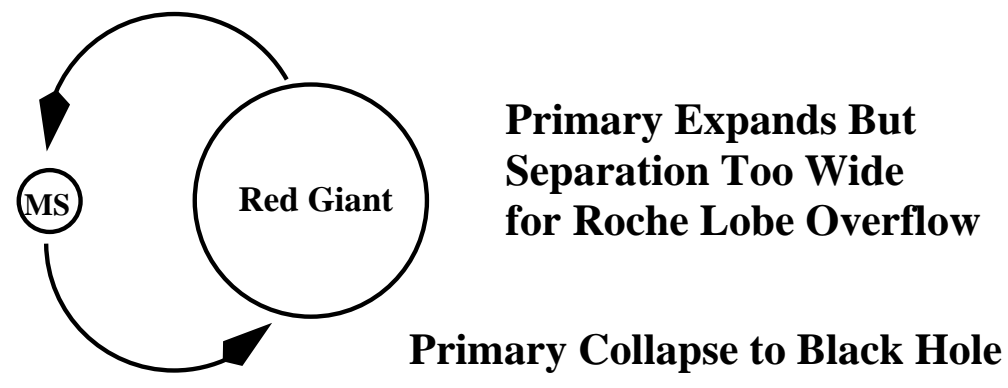

Kick Creates Short-Period Orbit

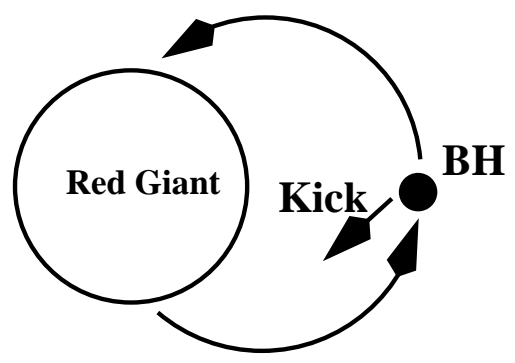

Secondary Evolves off

Main Sequence.

Possible Wind-Fed

X-ray Binary Phase

Secondary Sheds Mass and Becomes a White Dwarf

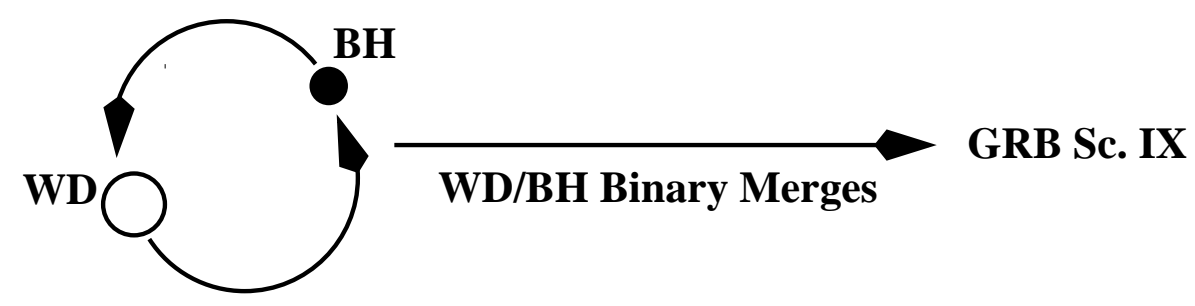

Fig. 10.- Scenario IX: The kick scenario for WD/BH binaries. Identical to scenario III (Fig. 4) except that the primary forms a black hole and the secondary forms a white dwarf. 


\section{Scenario X: Collapsar}

Initial Conditions:

(MS)

Single Star

$\mathbf{M}_{\text {star }}>M_{\text {BH }}$

Star Evolves of Main Sequence

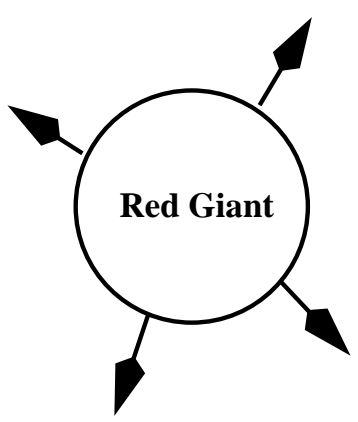

Wind Ejects Hydrogen

Envelope

He) $\stackrel{\text { He Core Collapses }}{\longrightarrow}$ GRB Sc. $X$

Fig. 11. - Scenario X: The single star Collapsar formation scenario. Wolf-Rayet winds blow off the hydrogen mantle of a rotating massive star leaving behind a massive helium core which then collapses to a black hole. The helium core must have a mass $\gtrsim 10 M_{\odot}$ to insure that the core immediately forms a black hole without any supernova explosion. 


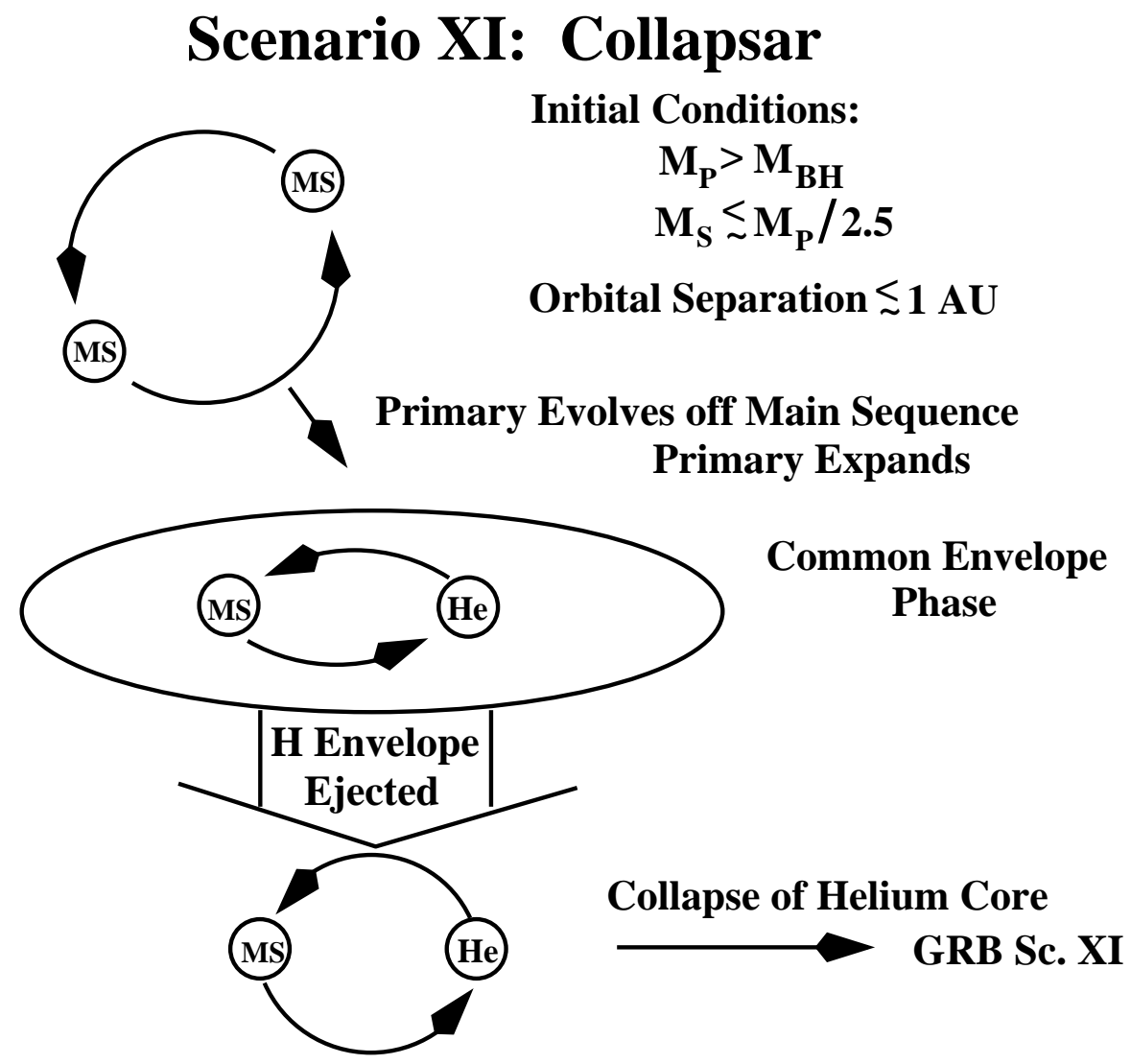

System May Evolve to BH/NS or WD/BH Binary

Fig. 12. - Scenario XI: The dominant collapsar formation scenario. Common envelope evolution drives off the hydrogen mantle of a rotating massive star. The helium core collapses to form a GRB. The system may then go on to form a WD/BH or $\mathrm{BH} / \mathrm{NS}$ binary. 


\section{Scenario XII: Collapsar}

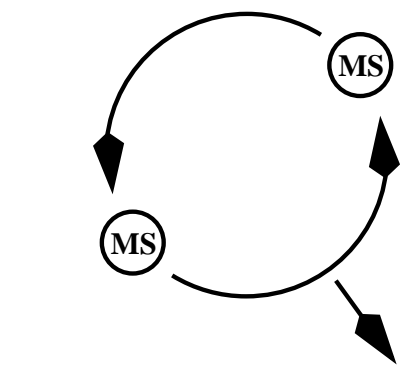

\section{Initial Conditions:}

$$
\begin{aligned}
& M_{P}>M_{B H} \\
& M_{S} \approx 0.95 M_{P}
\end{aligned}
$$

Orbital Separation $\lesssim 1 \mathrm{AU}$

Primary Evolves off Main Sequence

Primary Expands

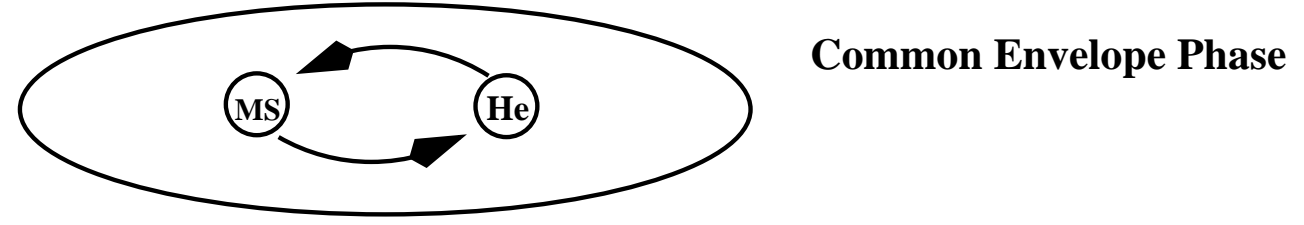

Secondary Evolves Off Main Sequence
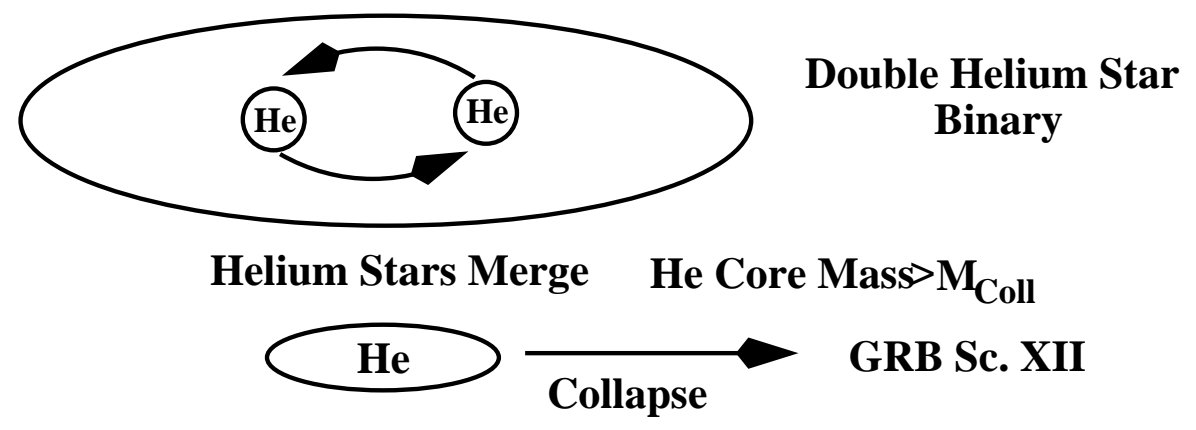

Fig. 13. - Scenario XII: collapsar scenario from the merger of a double helium binary. This model follows the same path as scenario II (Fig. 3) except that the helium stars merge, producing a flattened helium core which then powers the collapsar. 

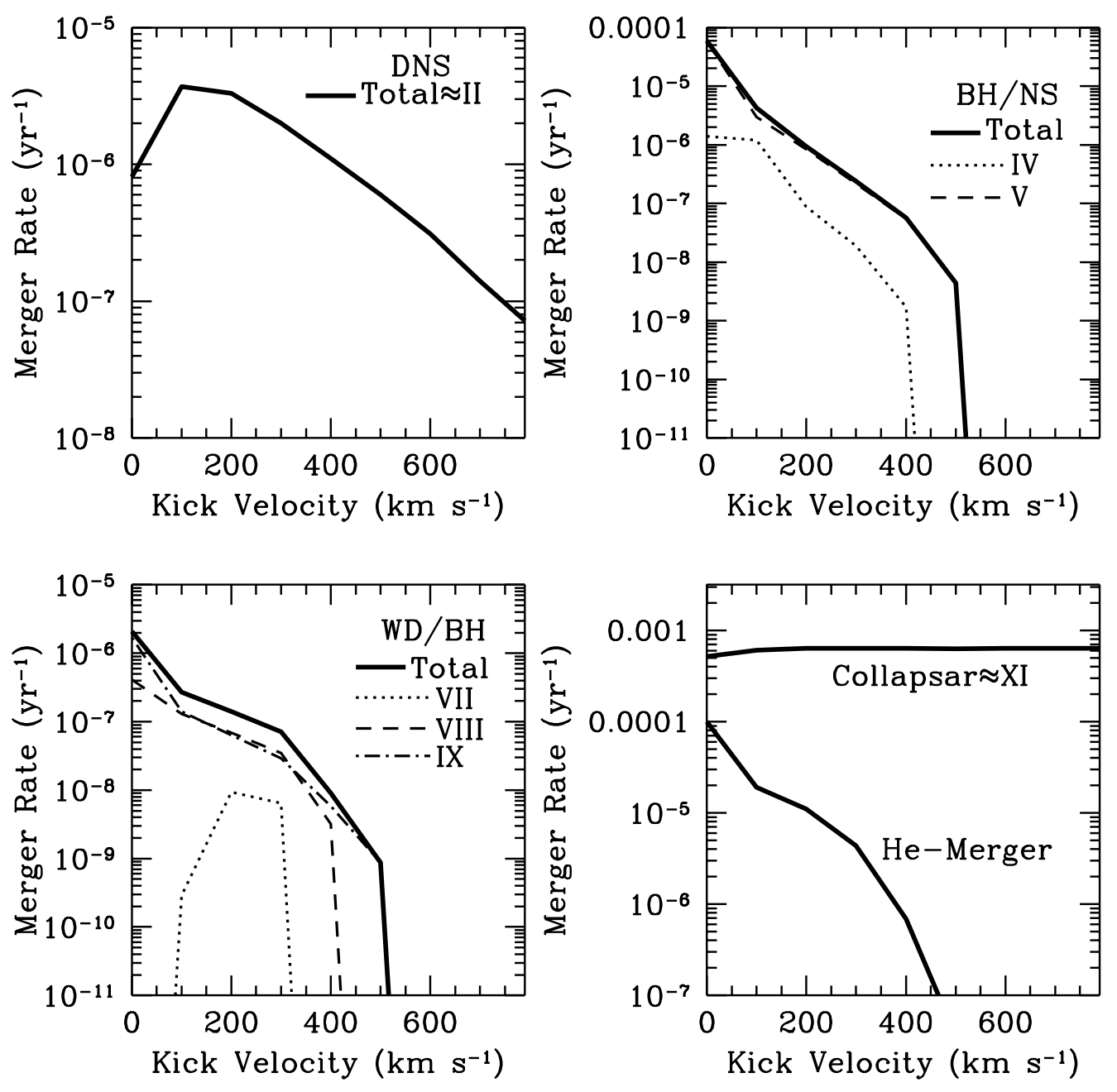

Fig. 14.- Merger rates of the various GRB systems vs. kick magnitude for isotropic kicks with delta function velocities. In the standard set of parameters, Scenario I is not allowed because hypercritical accretion converts such systems into BH/NS binaries via scenario V. Kicks strongly effect the black hole binaries, but primaries which collapse to form black holes may receive lower kicks. The drop at low velocities of the DNS mergers is caused because kicks are actually required to produce short-period (merging) binaries. Collapsars are not affected by the kick as most collapsars are produced in the collapse of the primary or in single systems. 

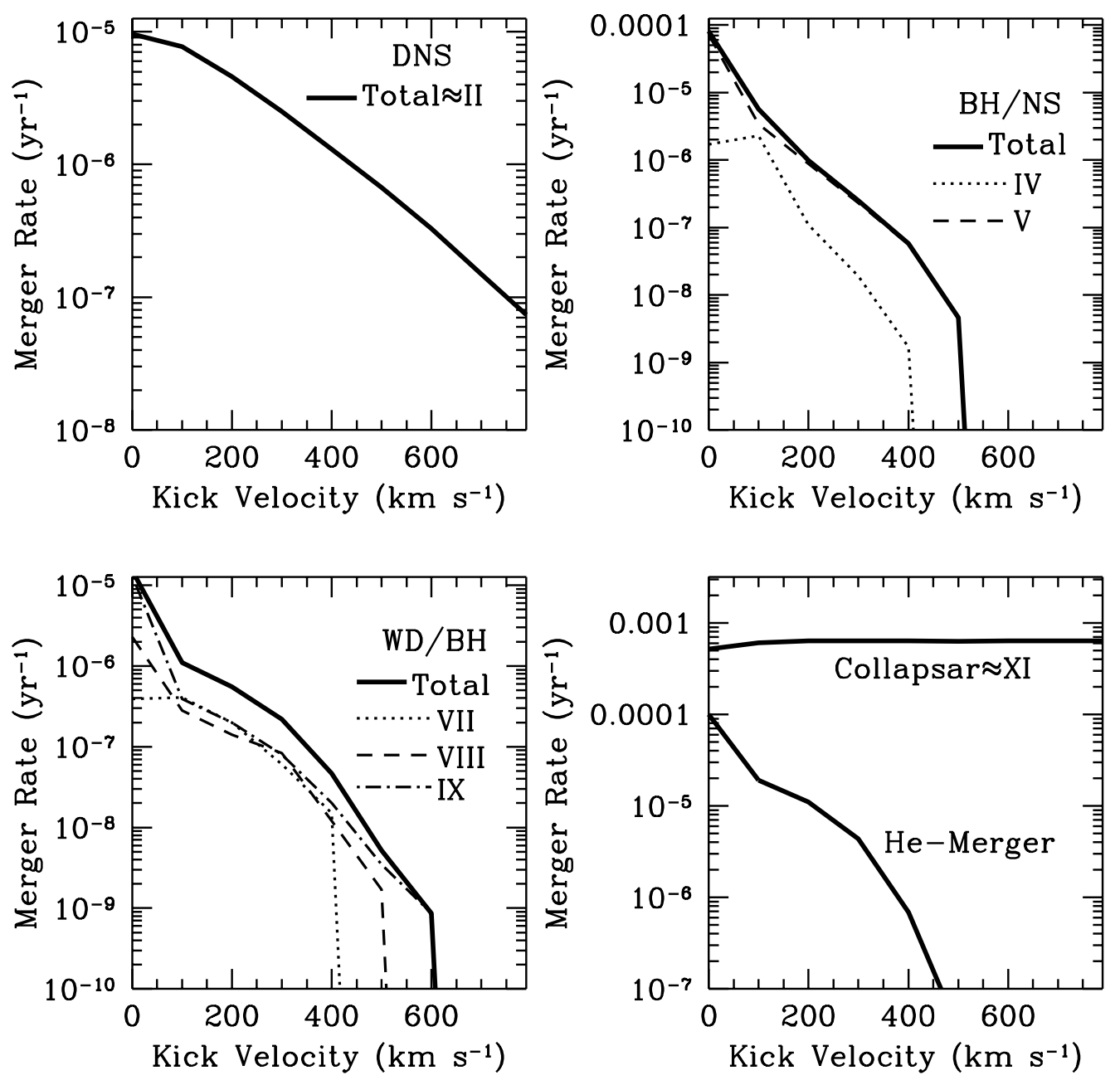

Fig. 15.- Formation rates (rather than merger rates) vs. kick velocity from Fig. 14. Note that the merger rate can be nearly an order of magnitude less than the formation rate for the binary mergers. 

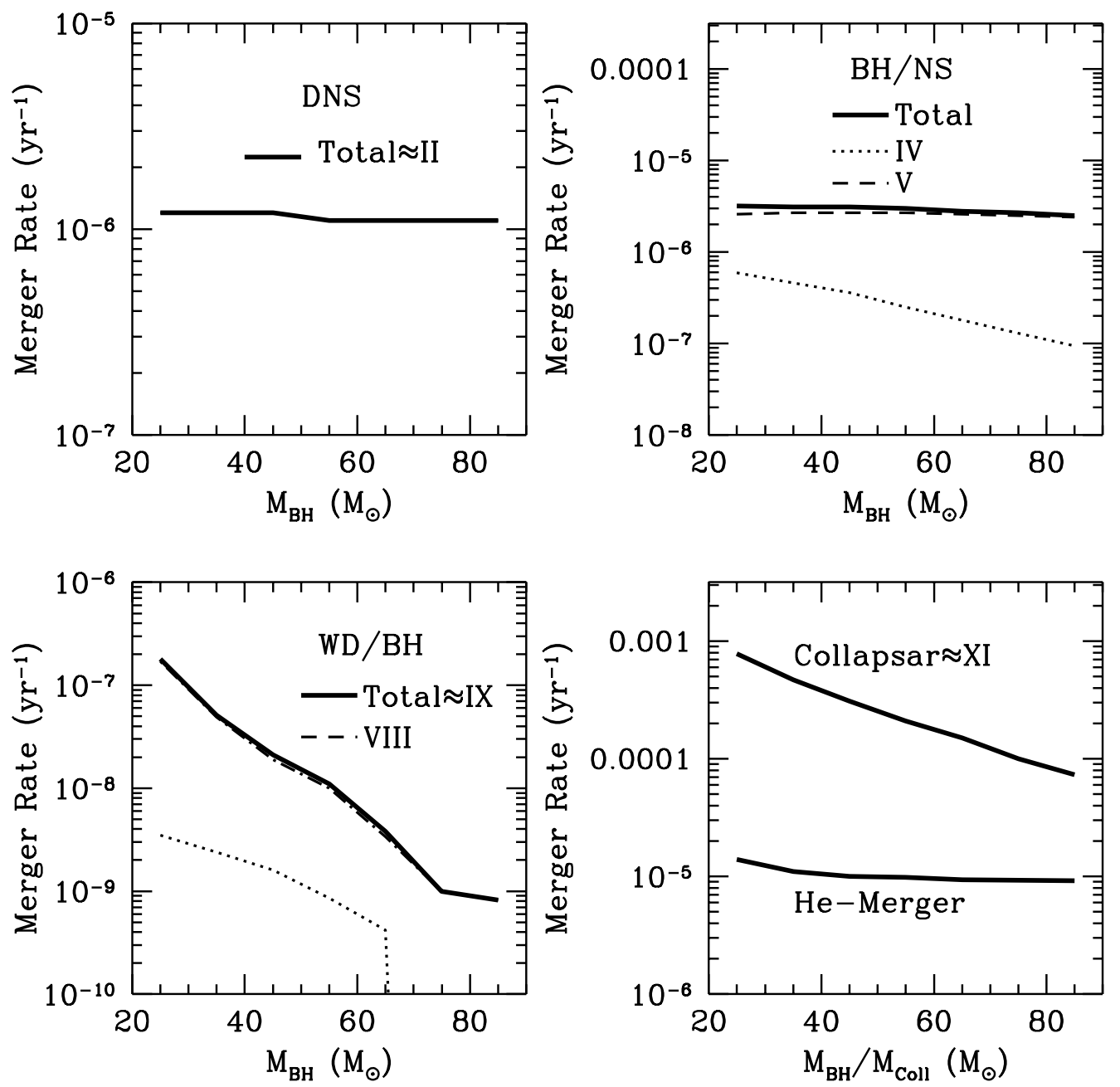

Fig. 16. - Merger rates vs. critical black hole mass using the FBB kick distribution ( $40 \%$ of neutron stars given kicks with $V_{\text {mean }}=100 \mathrm{~km} \mathrm{~s}^{-1}$ and $60 \%$ with $\left.V_{\text {mean }}=600 \mathrm{~km} \mathrm{~s}^{-1}\right) . M_{\text {Coll }}=M_{\mathrm{BH}}+15 M_{\odot}$. Note that if hypercritical accretion does not occur, the $\mathrm{BH} / \mathrm{NS}$ binary merger rate decreases rapidly with increasing critical mass. 


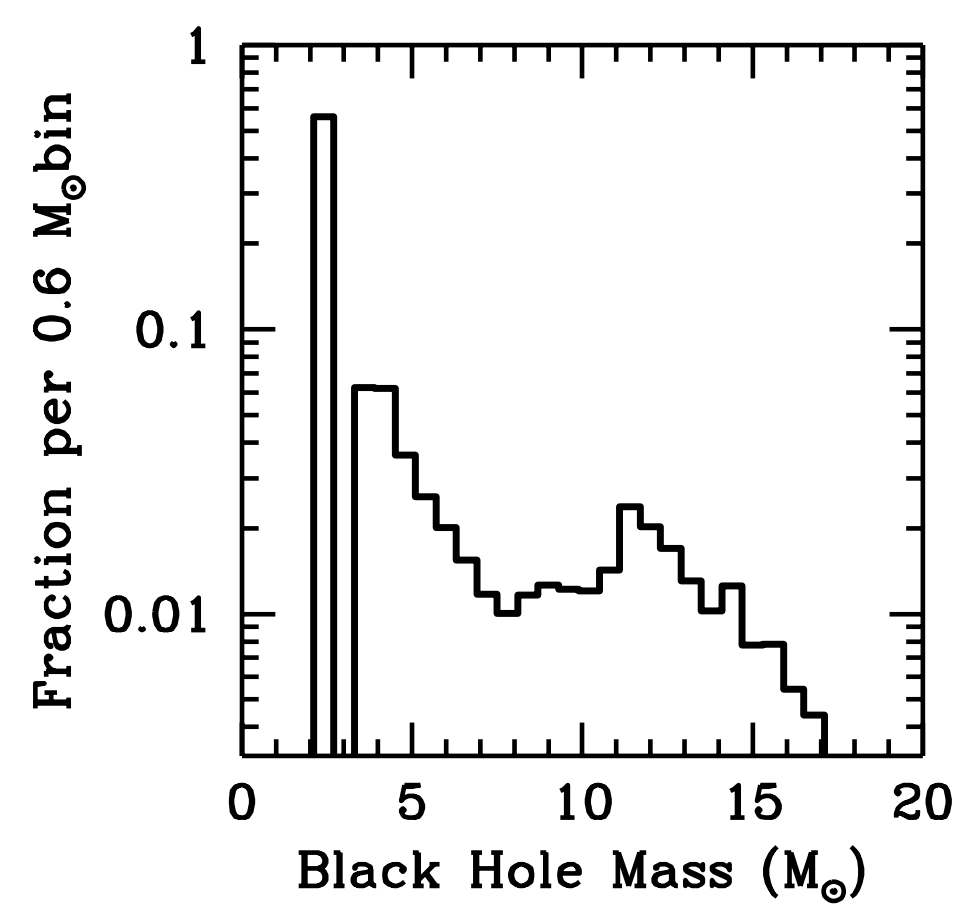

Fig. 17.- Mass distribution of black holes in $\mathrm{BH} / \mathrm{NS}$ binaries assuming the black hole mass is set to $1 / 3$ the mass of its progenitor at the time of collapse. The peak at $2.4 \mathrm{M}_{\odot}$ is due to those neutron stars which collapse to form black holes after accreting $\sim 1 \mathrm{M}_{\odot}$ in a common envelope phase. 

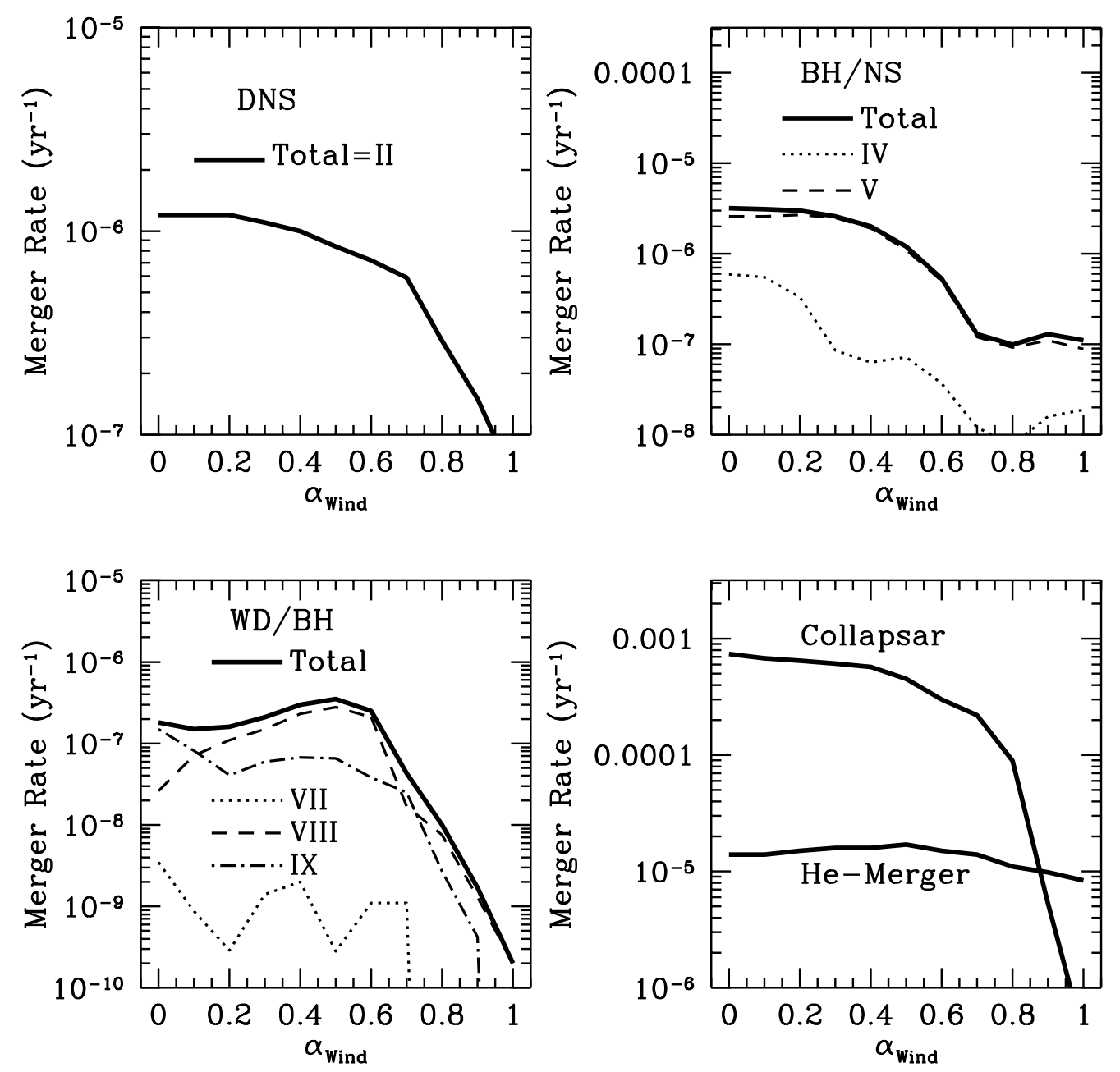

Fig. 18. - Merger rates vs. mass loss fraction $\alpha_{\text {Wind }}=($ Mass Loss $) /($ Mass LosswLW $)$. The Collapsar rate depends most sensitively upon the mass loss rate, but for the range of reliable values for $\alpha_{\text {Wind }}(0 .-0.5)$, the dependence is slight. However, if one uses the full Mass Loss of Woosley, Langer, \& Weaver $(1993,1995)$, the collapsar and BH/NS GRB rates decrease dramatically (Table 4). 

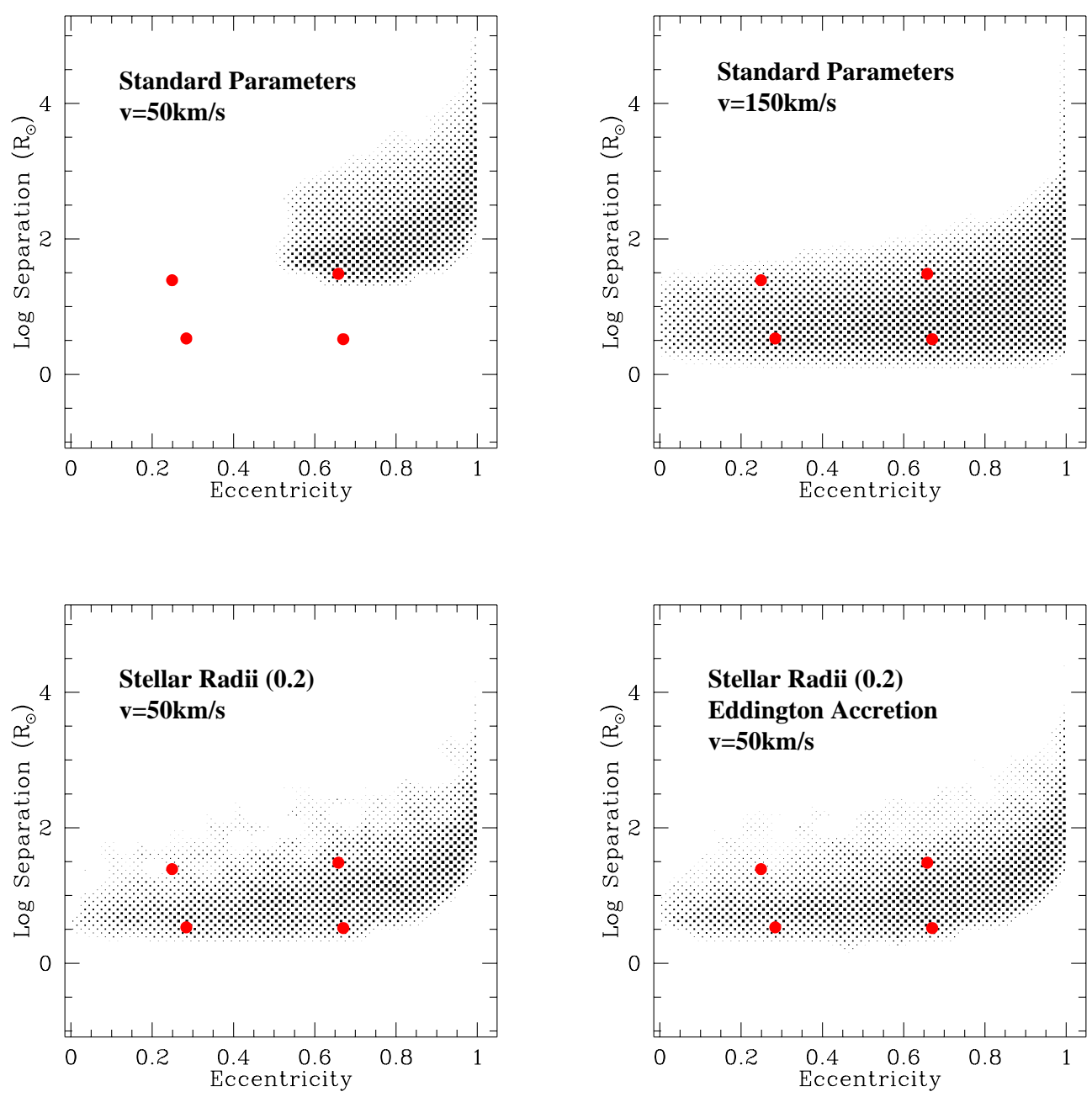

Fig. 19.- Separation/eccentricity distribution of DNS binaries compared to the 4 observed galactic binaries. Although we can constrain some sets of parameter choices, we do not constrain the allowed parameter space enough to severely limit the uncertainties. 

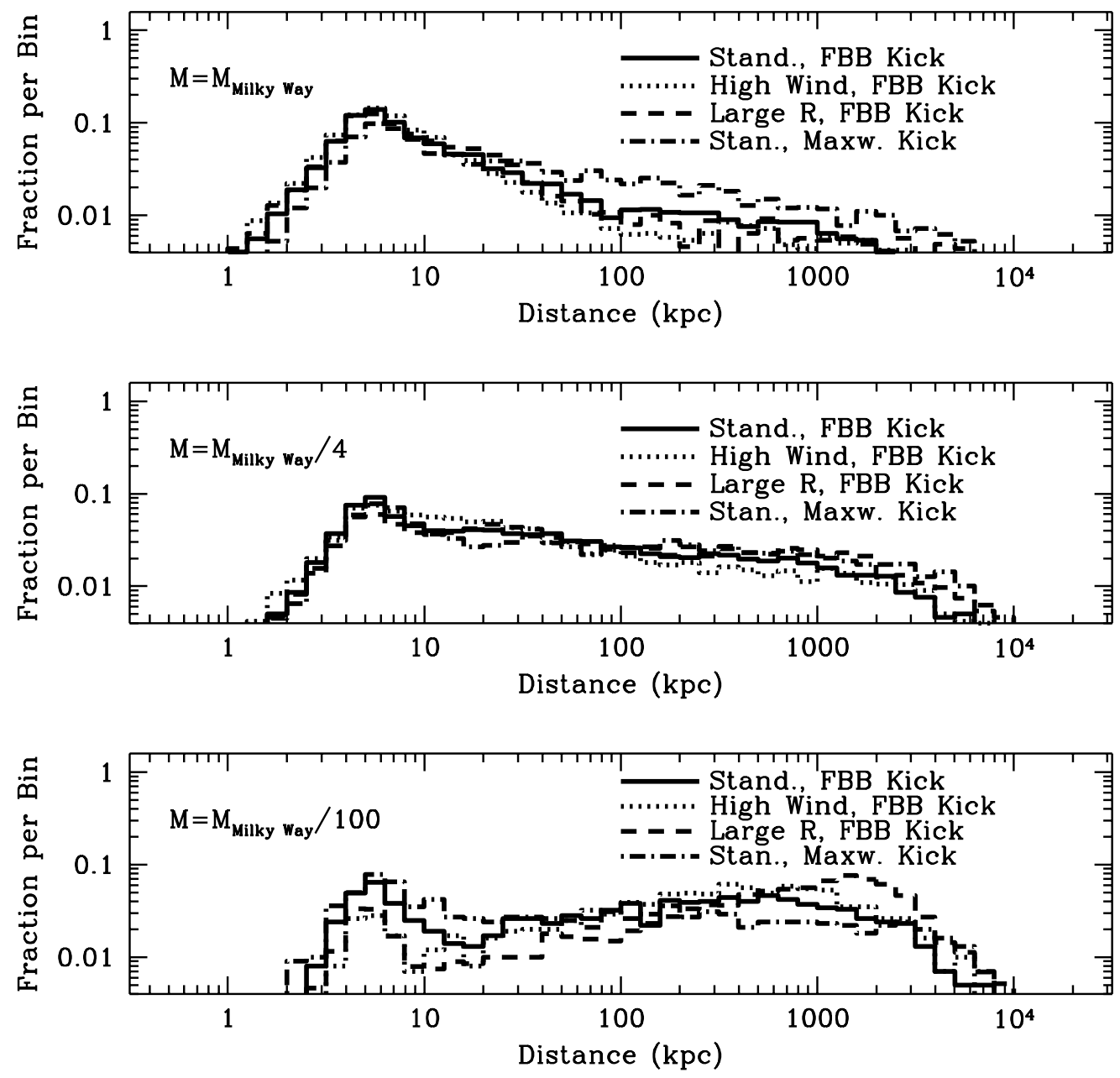

Fig. 20.- Distribution of distance from the host galaxy for DNS and BH/NS binaries using a range of population synthesis parameters for (a) a galaxy mass equal to that of the Milky Way and (b) a galaxy mass set to 1/4th that of the Milky Way. A distance of $100 \mathrm{kpc}$ corresponds to roughly 20 arc seconds at a redshift of 1-3. 


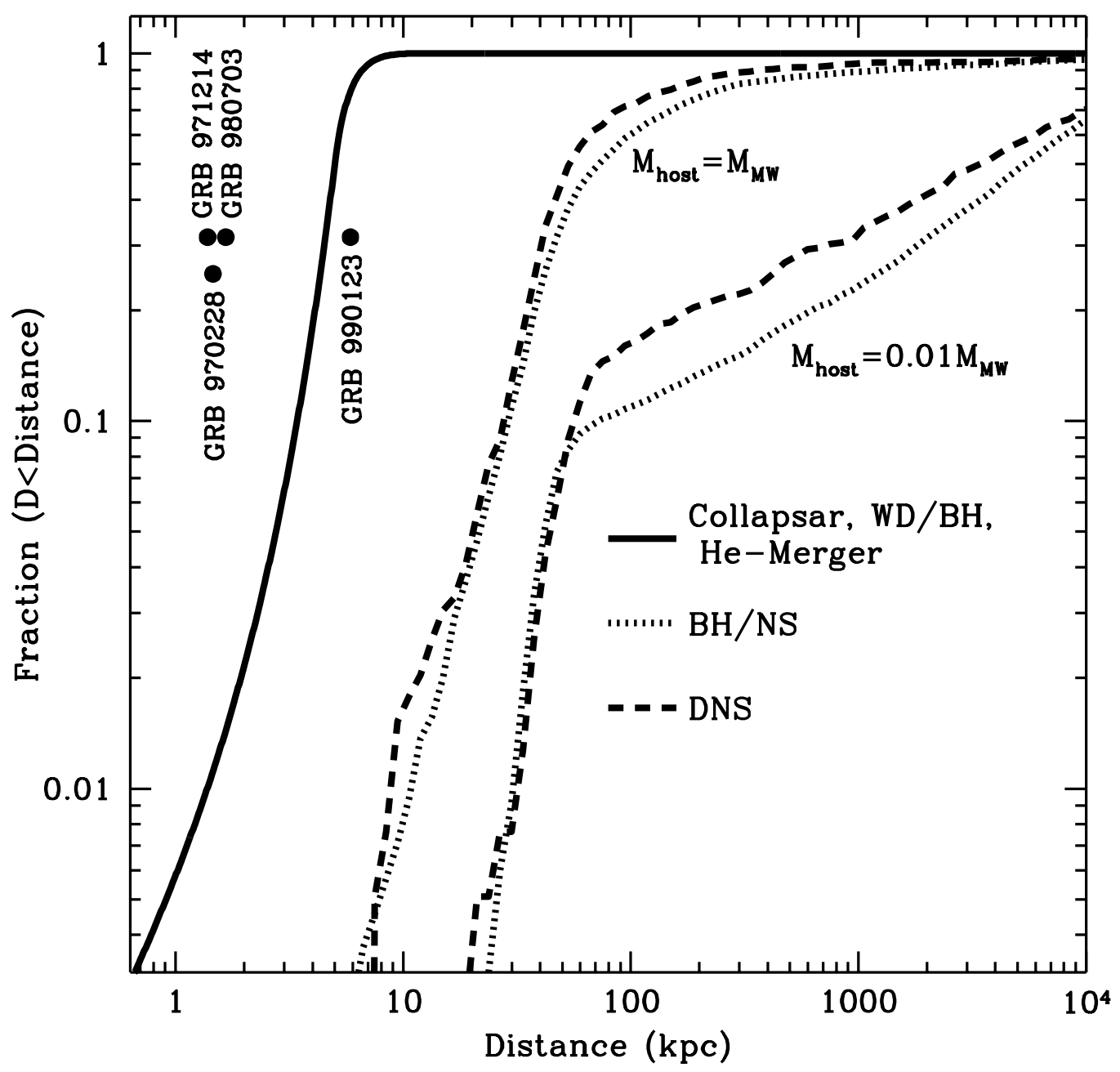

Fig. 21. - The fraction of GRBs within a given distance of their host galaxy using the standard set of population synthesis parameters with large radii and the FBB kick distribution. Note that the DNS and $\mathrm{BH} / \mathrm{NS}$ mergers occur at distances well beyond the observed long-duration bursts. The distribution of $\mathrm{WD} / \mathrm{BH}$ mergers, collapsars and helium-mergers trace our distribution of star formation regions. The separations of the localized GRBs from the center of the host galaxy are given for comparison assuming $h_{0}=65 \mathrm{~km} \mathrm{~s}^{-1} \mathrm{Mpc}^{-1}$ and $q_{0}=0$. . We do not include GRB 970508 which was at the host center). 


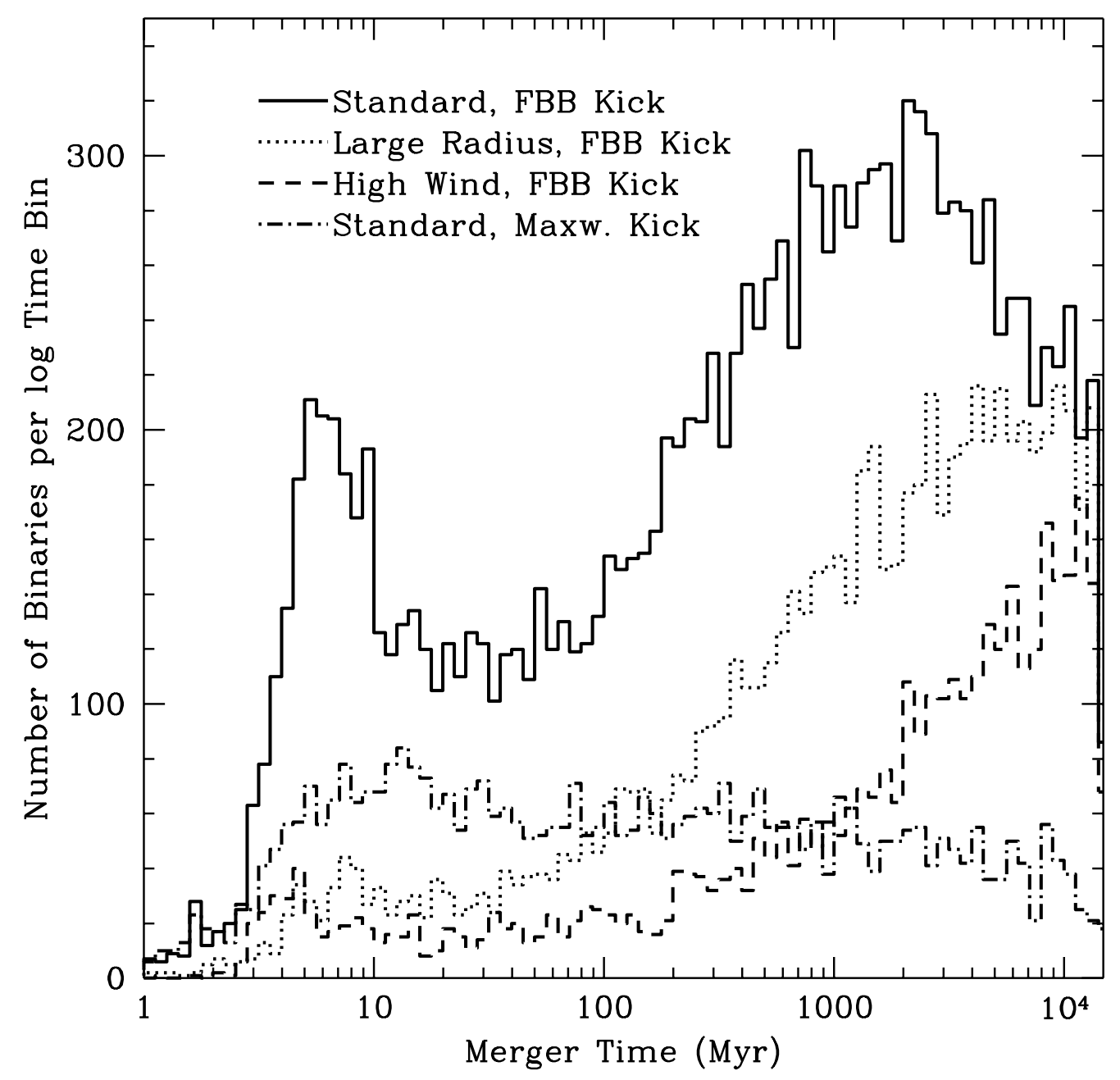

Fig. 22.- Merger times for DNS and BH/NS binaries using a range of population synthesis parameters. Higher stellar radii or winds produce wider binaries and hence, longer merger times. The double peaked merger distribution for the standard model (solid line) is a signature of the double peaked FBB kick distribution. $>90 \%$ of the binaries with merger times less than $30 \mathrm{Myr}$ received kicks of $300 \mathrm{~km} \mathrm{~s}^{-1}$ or greater. 

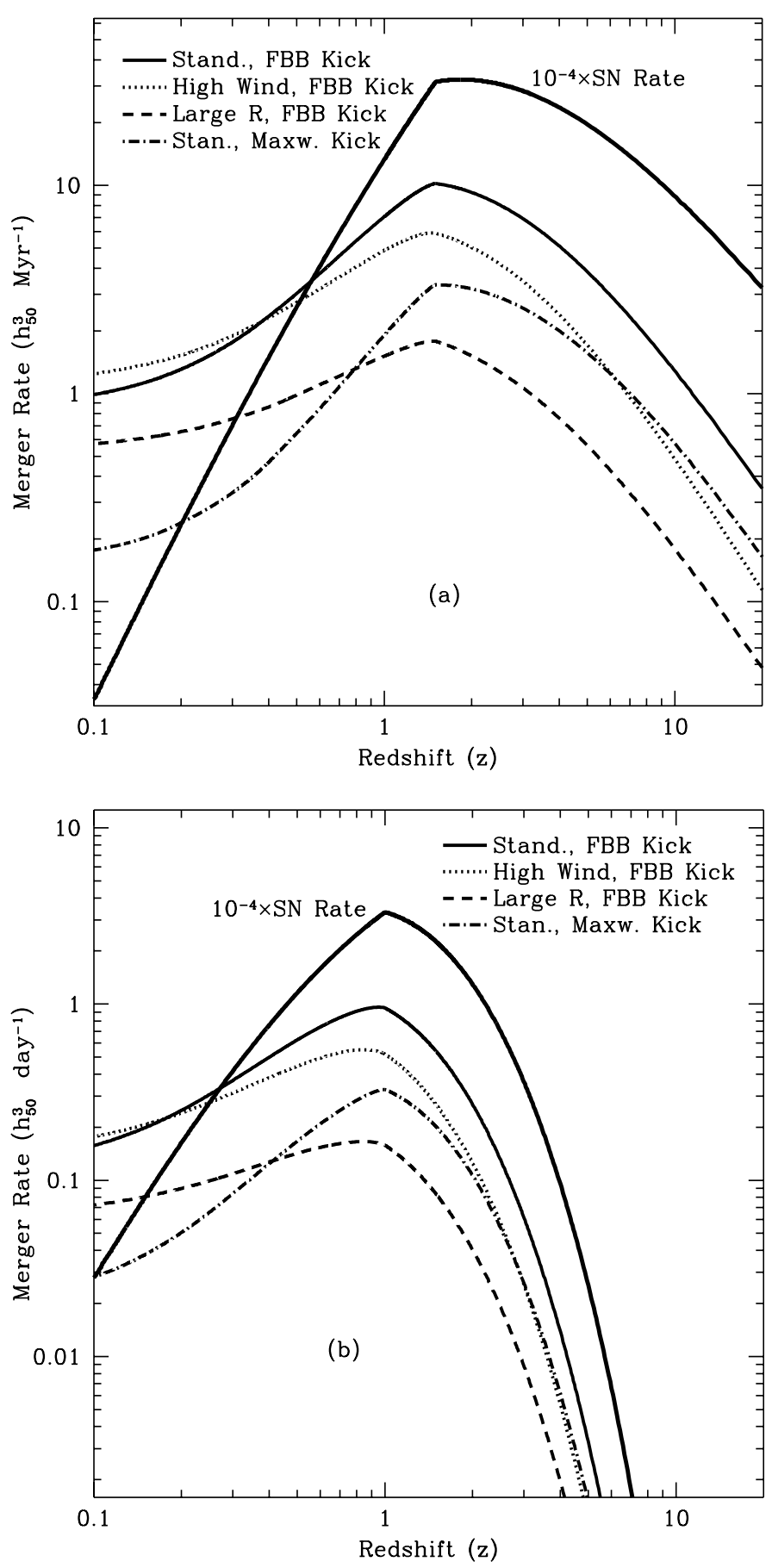

Fig. 23.- The redshift distribution of the merger rate of DNS and BH/NS binaries for an Einstein-deSitter universe and three different star formation histories: a) flattened star formation rate - $\mathrm{A}=3.0, \mathrm{~B}=0.0, \mathrm{z}_{\mathrm{p}}=1.5$; b) peaked star formation rate $-\mathrm{A}=1.0, \mathrm{~B}=0.5, \mathrm{z}_{\mathrm{p}}=1.0$. The star formation history is proportional to the supernova history (thick solid line). The merger times cause a delay in the bursts produced by these mergers. The median redshift of DNS and BH/NS GRBs is much less than that of supernovae. The median redshift of $\mathrm{WD} / \mathrm{BH}$ mergers, helium-mergers, and collapsars are all roughly equal to the supernova median redshift. 